\title{
Jeu, \\ simulation \\ et jeu de rôle
}

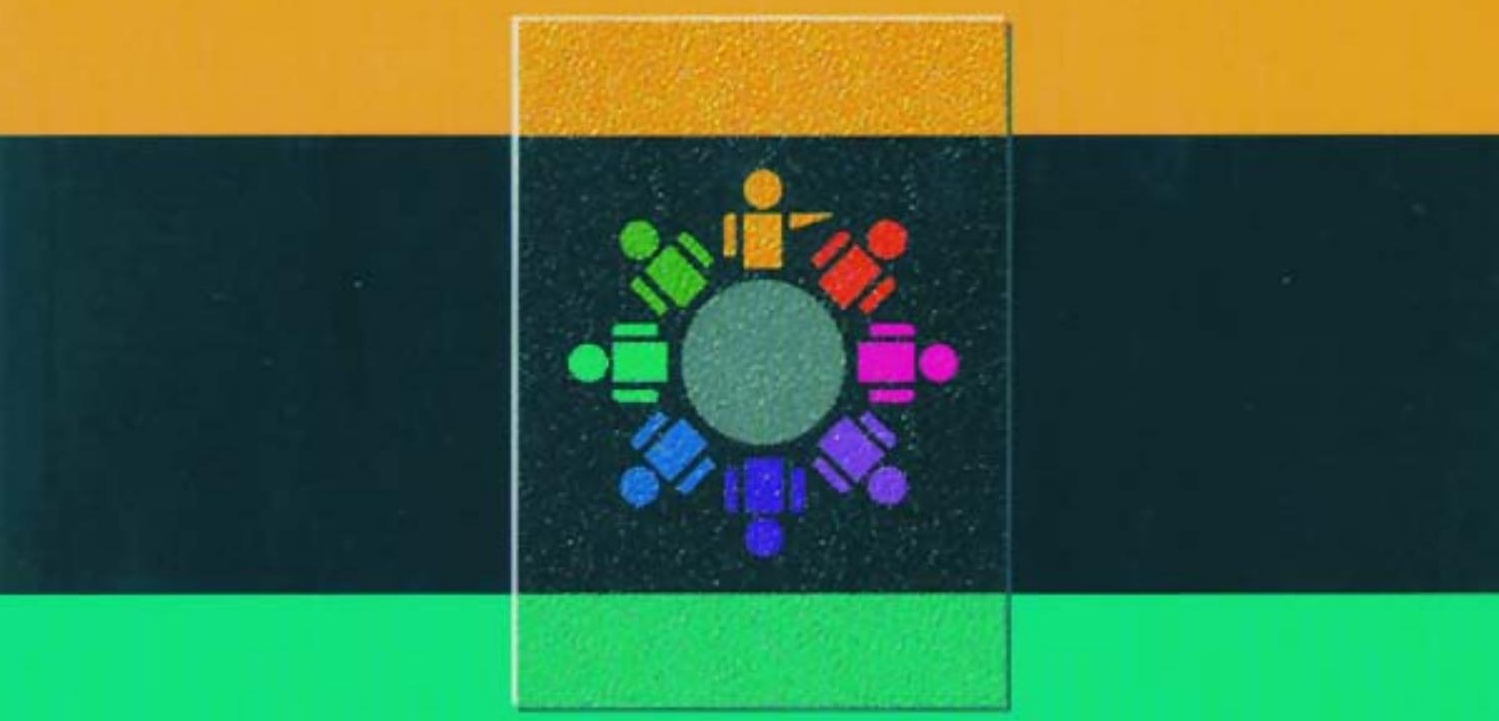

\section{Gilles Chamberland}

\section{et \\ Guy Prowast}





\section{Jeu, \\ simulation \\ et jeu de rôle}




\section{DANS LA MÊME COLLECTION}

\section{0 formules pédagogiques}

Gilles Chamberland, Louisette Lavoie et Danielle Marquis 1995, 192 pages

PRESSES DE L'UNIVERSITÉ DU QUÉBEC

2875, boul. Laurier, Sainte-Foy (Québec) GIV 2M3

Téléphone : (418) 657-4399

Télécopieur : (418) 657-2096

Catalogue sur Internet : http://www.uquebec.ca/puq/puq.html

\section{Distribution :}

DISTRIBUTION DE LIVRES UNIVERS S.E.N.C.

845, rue Marie-Victorin, Saint-Nicolas (Québec) G0S 3L0

Téléphone: (418) 831-7474 / 1-800-859-7474

Télécopieur: (418) 831-4021

Europe :

ÉDITIONS ESKA

27, rue Dunois, 75013. Paris, France

Téléphone: (1) 45836202

Télécopieur: (1) 44240694 


\title{
Jeu, \\ simulation \\ et jeu de rôle
}

Gilles Chamberland

\author{
et \\ Guy Provost
}


Données de catalogage avant publication (Canada)

Chamberland,Gilles, 1957-

Jeu, simulation et jeu de rôle

(Collection Formules pédagogiques)

Comprend des réf. bibliogr.

ISBN 2-7605-0894-3

1. Jeux éducatifs. 2. Jeux de simulation en éducation. 3. Jeu de rôle. 1. Provost, Guy, 1945- . II. Titre. III. Collection.

LB1029.G3C42 $1996 \quad 371.3^{\prime} 078 \quad$ C96-940845-5

Révision linguistique: GISLAINE BARETTE

Mise en pages: INFO 1000 MOTS INC.

Conception graphique de la couverture : CARON \& GOSSELIN COMMUNICATION GRAPHIQUE

\section{PUQ 1996987654321}

Tous droits de reproduction, de traduction et d'adaptation réservés C 1996 Presses de l’Université du Québec

Dépôt légal $-3^{\mathrm{e}}$ trimestre 1996

Bibliothèque nationale du Québec / Bibliothèque nationale du Canada Imprimé au Canada 


\section{Avant-propos}

La collection Formules pédagogiques regroupe un ensemble d'ouvrages portant sur vingt formules pédagogiques parmi les plus utiles en enseignement.

L'ouvrage de base de la collection s'intitule 20 formules pédagogiques. Il présente les éléments essentiels à connaître avant de privilégier une formule plutôt qu'une autre et procède ensuite à la présentation des principales caractéristiques de chacune d'entre elles.

Chacun des autres ouvrages porte sur une formule pédagogique particulière, ou sur plus d'une formule si certains aspects qui les caractérisent permettent de les rassembler. Ces ouvrages spécialisés abordent chacune de ces formules d'une façon plus approfondie et plus complète que ne le fait l'ouvrage de base.

C'est la typologie présentée dans $\mathbf{2 0}$ formules pédagogiques qui sert de fil conducteur aux autres ouvrages de la collection. Cette typologie est fondée sur trois dimensions importantes de l'acte d'enseigner: le degré de contrôle de l'apprentissage, l'organisation du groupe et l'utilisation de médias. 
Dans cette typologie, chacune de ces trois dimensions comprend deux pôles qui s'opposent et entre lesquels des positions intermédiaires sont possibles selon les caractéristiques de chacune des formules.

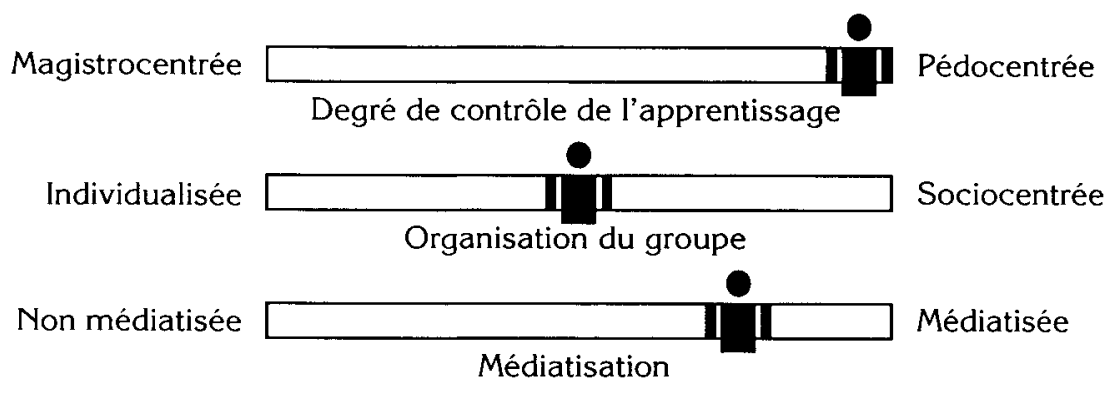

Ainsi, pour ce qui est de la dimension degré de contrôle de l'apprentissage, une formule pédagogique est qualifiée de magistrocentrée si le plein contrôle de l'apprentissage est exercé par l'enseignant; si, au contraire, l'initiative revient davantage à l'apprenant, il s'agit alors d'une formule pédocentrée.

Quant à la dimension organisation du groupe, une formule est dite individualisée lorsque l'apprenant est autonome, travaille à son rythme et qu'il y a peu d'échanges avec l'ensemble du groupe; à l'opposé, une formule caractérisée par l'utilisation du groupe comme moteur de l'apprentissage correspond à une formule sociocentrée.

Pour ce qui est de la dimension médiatisation, une formule est qualifiée de non médiatisée ou de médiatisée selon l'absence ou la présence plus ou moins importante d'intermédiaires entre l'enseignant et l'apprenant. 
Jeu, simulation et jeu de rôle, est le premier des ouvrages spécialisés de la collection. Ces trois formules ont été regroupées parce que plusieurs de leurs caractéristiques peuvent être traitées en commun. Voilà certes trois formules fort intéressantes à explorer pour l'enseignant qui veut favoriser l'interactivité en classe. Les auteurs ont donné à leur ouvrage une orientation résolument pratique en fournissant plusieurs exemples concrets et en utilisant généreusement les tableaux et les figures, ce qui a pour effet de faciliter grandement la compréhension des concepts présentés.

En tant que directeurs de la collection, nous souhaitons au lecteur beaucoup de plaisir à découvrir ou à redécouvrir ces trois formules pédagogiques parmi les mieux adaptées aux défis de l'enseignement moderne. Nous les encourageons à en faire profiter pleinement les premiers bénéficiaires de toute entreprise éducative: les apprenants.

Nous apprécierions que des lecteurs et des utilisateurs nous fassent part de leurs commentaires, suggestions, exemples, etc. qui pourraient contribuer à augmenter et à améliorer le contenu des éditions ultérieures.

Les directeurs de la collection

Gilles Chamberland

Louisette Lavoie

Danielle Marquis 



\section{Table des matières}

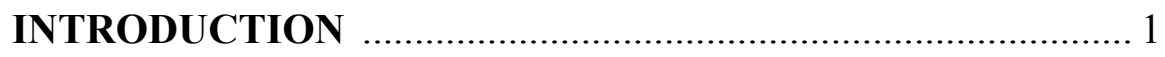

STRUCTURE DE L'OUVRAGE …………………………....... 3

CHAPITRE 1 SURVOL HISTORIQUE …………................. 5

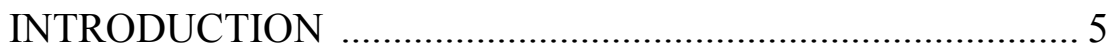

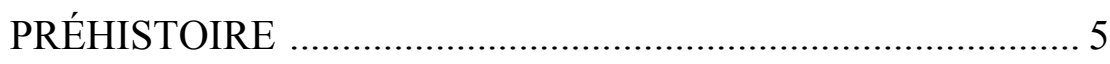

ANTIQUITÉ ET MOYEN ÂGE …………………………....... 8

DE LA RENAISSANCE AU XX ${ }^{\mathrm{e}}$ SIÈCLE ............................... 12

CHAPITRE 2 FONDEMENTS PÉDAGOGIQUES .................. 19

UNE RÉACTION À L'ENSEIGNEMENT

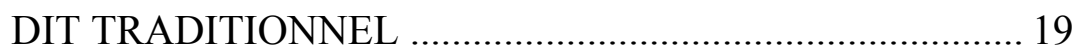

UNE VISION DES COMPÉTENCES NÉCESSAIRES

DANS LE FUTUR …………………………………...... 21

UNE CONCEPTION PARTICULIÈRE

DE L'ENSEIGNEMENT

23 
Jeu, simulation et jeu de rôle

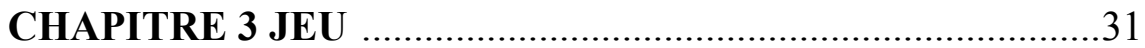

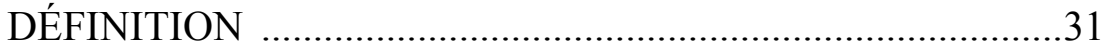

SITUATION TYPOLOGIQUE DU JEU ………………….........33

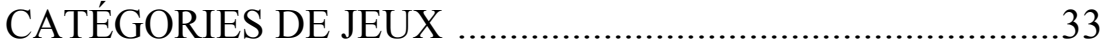

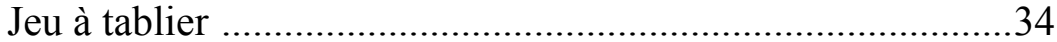

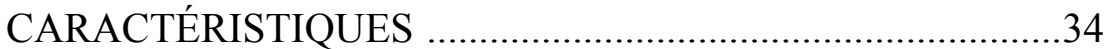

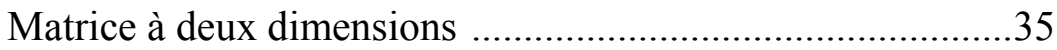

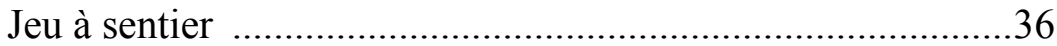

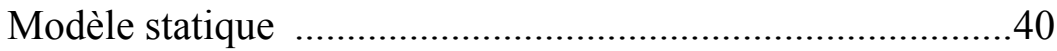

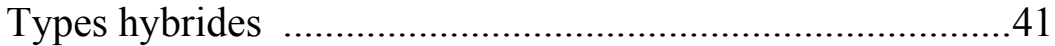

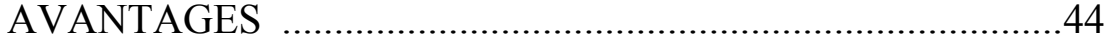

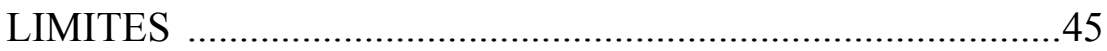

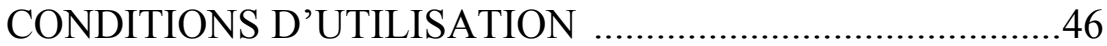

EXEMPLES DE JEUX …………………………………........48

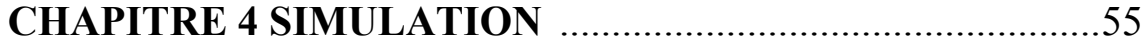

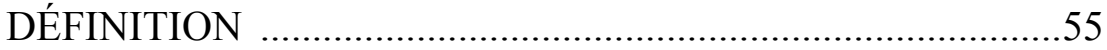

SITUATION TYPOLOGIQUE DE LA SIMULATION ...........56

CARACTÉRISTIQUES …………………………….............56

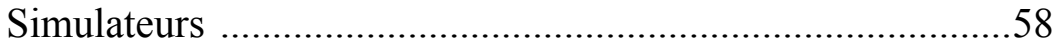

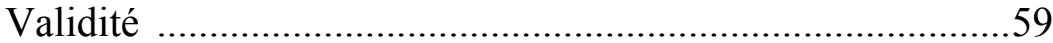

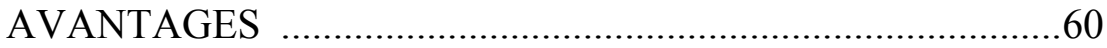

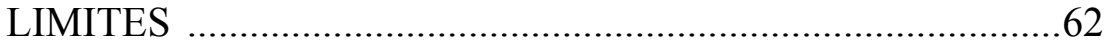

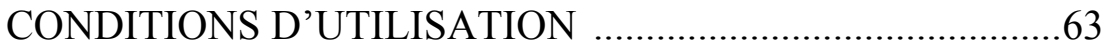

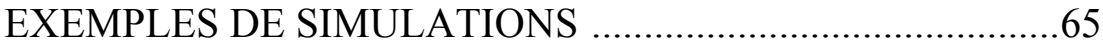

CHAPITRE 5 JEU DE RÔLE ...................................................

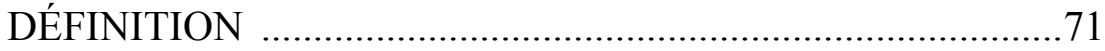

SITUATION TYPOLOGIQUE DU JEU DE RÔLE ..................72 
ORIGINES DU JEU DE RÔLE ............................................ 72

Le cas Barbara ................................................................... 74

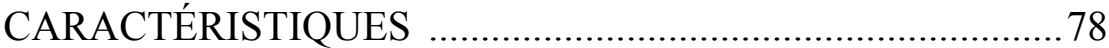

Acquisition d'habiletés techniques et de méthodes ................79

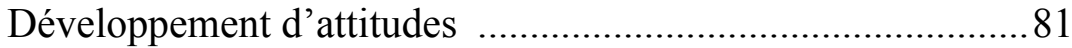

Compréhension de faits et de principes ...............................83

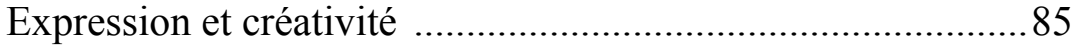

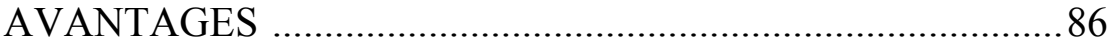

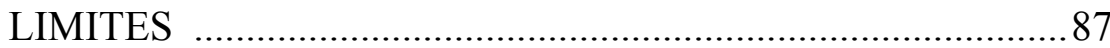

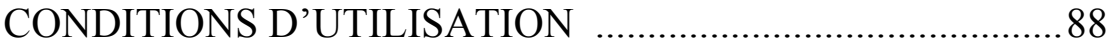

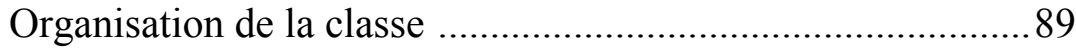

EXEMPLES DE JEUX DE RÔLE ..........................................92

CHAPITRE 6 CONCEPTION DE JEUX, SIMULATIONS ET JEUX DE RÔLE .............105

INTRODUCTION ............................................................... 105

MODĖLE DE CONCEPTION DE JEUX,

SIMULATIONS ET JEUX DE RÔLE ..................................105

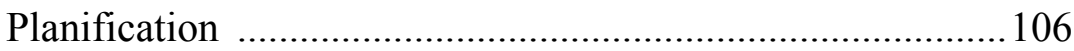

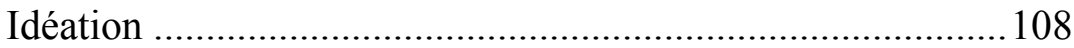

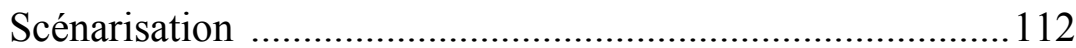

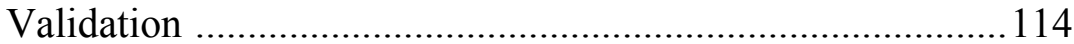

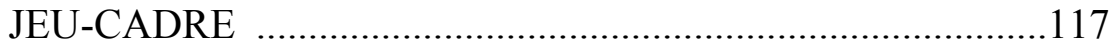

Structure et contenu :

deux éléments fondamentaux d'un jeu ............................. 118

Plusieurs types et niveaux d'adaptation ................................ 120

À éviter avec les jeux-cadres ................................................. 123

QUELQUES CONSEILS PRATIQUES

POUR LA PRODUCTION D'UN JEU À TABLIER ......... 124 
Jeu, simulation et jeu de rôle

\section{CHAPITRE 7 PRÉPARATION, ANIMATION}

ET EXPLOITATION DES SIMULATIONS

ET DES JEUX DE RÔLE

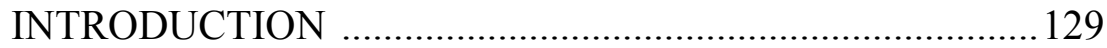

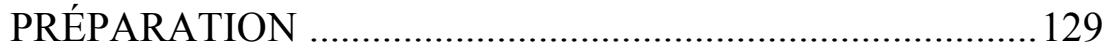

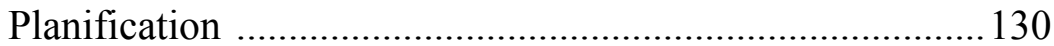

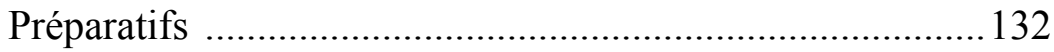

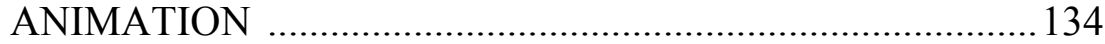

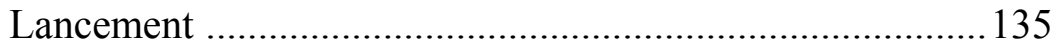

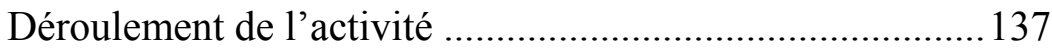

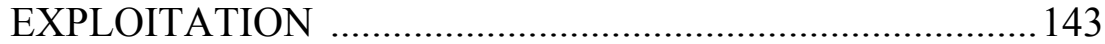

Debriefing .................................................................... 143

ÉVALUATION FORMATIVE DU JEU DE RÔLE

OU DE LA SIMULATION ............................................... 149

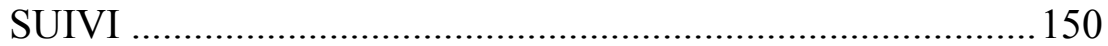

CHAPITRE 8 TÉMOIGNAGES D'ENSEIGNANTS ...........157

TÉMOIGNAGE D’UNE ENSEIGNANTE

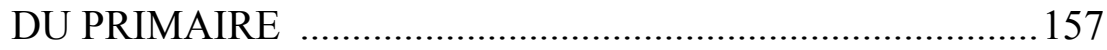

TÉMOIGNAGE D’UNE ENSEIGNANTE

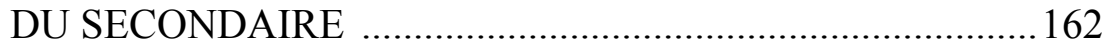

TÉMOIGNAGE D’UN ENSEIGNANT

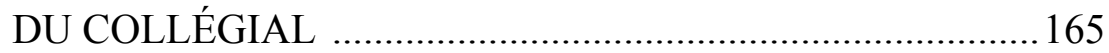

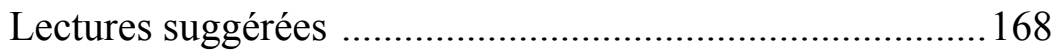

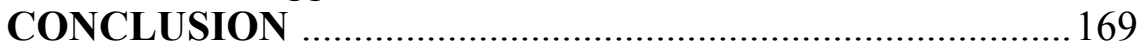

BIBLIOGRAPHIE ....................................................... 171

INDEX DES JEUX, SIMULATIONS ET JEUX DE RÔLE.. 177 


\section{Liste des tableaux}

Tableau 1 Comparaison entre l'apprentissage déductif et l'apprentissage inductif (adapté de Heinich, Molenda et Russell, 1989, p. 311) ................................28

Tableau 2 Comparaison des trois types de jeux à tablier

Tableau 3 Exemple d'ordre de substitution des joueurs dans le jeu de rôle Bonhomme-bonhomme 99

Tableau 4 Liste de vérification pour la préparation, l'animation et l'exploitation pédagogique d'un jeu de rôle ou d'une simulation 154 


\section{Liste des figures}

Figure 1 Le concept de jeu de simulation ......................................... 2

Figure 2 Mancala ou Wari …………………………....................

Figure 3 Les échecs et les échecs chinois ........................................ 9

Figure 4 Le jeu de go ................................................................. 11

Figure 5 Structure de base du jeu de l'oie ......................................14

Figure 6 Modèle de l'apprentissage expérientiel de Kolb (traduit de Kolb, Rubin et Osland, 1991, p. 59)

Figure 7 Continuum des stratégies de l'enseignant

(traduit de Reinhartz et Van Cleaf,

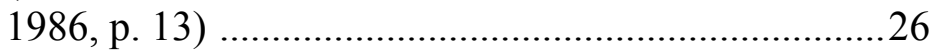

Figure 8 Les composantes d'un jeu ..............................................32

Figure 9 Quatre types de jeux à sentier ........................................... 37

Figure 10 Exemple de sentier à une seule piste avec raccordements : Narcisse et Pivoine sur le chemin des floralies

Figure 11 Exemple d'un jeu de type modèle statique : «Risk »

Figure 12 Relation entre le réalisme de la simulation et l'efficacité de l'enseignement (Norris, 1986, p. 449) 60 
Figure 13 Territoires et populations tels qu'ils sont représentés dans « Répartition » avec 25 participants .66

Figure 14 Répartition de la richesse et de la nourriture tels qu'ils sont représentés dans « Répartition » avec 25 participants

Figure 15 Organisation physique de la classe pour le jeu de rôle selon les techniques « centralisée », de l' « aquarium », du « théâtre-forum et décentralisée

Figure 16 Exemple de cartes de style tirées de "Rencontres du $13^{\mathrm{e}}$ type »

Figure 17 Quelques-uns des personnages de la série « Bonhomme »

Figure 18 Carte géographique des environs de Sainte-Fictive tirée du jeu de rôle « Alerte à l'environnement »

Figure 19 Modèle de conception d'un jeu, d'une simulation ou d'un jeu de rôle

Figure 20 Composantes du jeu-cadre (adapté de Stolovitch, 1989)

Figure 21 Exemple d'application du bingo comme jeu-cadre : « Sagix »

Figure 22 Exemple d'application du tic-tac-tœ comme jeu-cadre : « Ça va vous tuer » (adapté de Stolovitch et Thiagarajan, 1980) 



\section{Introduction}

Le défi de tout enseignant ou formateur est de créer un environnement qui puisse favoriser les apprentissages.

Outre l'expertise qu'il possède dans le champ de connaissances qui fait l'objet d'un cours, l'intervenant en enseignement ou en formation doit maîtriser les modes et les stratégies de transmission du savoir et du savoir-faire. Bref, il doit maîtriser contenu et stratégie.

Le jeu, la simulation et le jeu de rôle sont des stratégies particulièrement intéressantes pour qui veut varier les approches, susciter l'intérêt de l'apprenant et l'engager davantage dans le processus d'enseignement-apprentissage. Parmi les nombreuses formules pédagogiques dont dispose l'enseignant, le jeu, la simulation et le jeu de rôle tiennent une place bien légitime et représentent d'heureux compléments aux formules d'usage courant. Tout au long de cet ouvrage, nous présentons leur contribution à l'enseignement et à la formation.

Soulignons que plusieurs définitions des concepts de jeu, de simulation et de jeu de rôle ont été formulées dans un nombre imposant d'ouvrages très variés. Une évidence ressort : l'absence de consensus. Il faut dire que ces formules pédagogiques ne sont pas aussi faciles à cerner qu'on pourrait le croire à première vue. 
Jeu, simulation et jeu de rôle

Il existe en effet une étroite parenté entre le jeu, la simulation et le jeu de rôle. C'est d'ailleurs la raison qui nous a poussés à les traiter conjointement dans cet ouvrage. Il y a aussi plusieurs zones grises, ce qui rend parfois difficile la classification d'une activité. Cependant, à notre avis, les nuances de catégorisation ont fort peu d'importance pour l'utilisateur.

Dans la littérature, les activités qui relèvent à la fois du jeu, de la simulation et du jeu de rôle (ou d'une quelconque combinaison de deux d'entre eux) sont désignées tantôt par le terme " simulation-

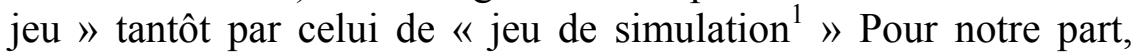
nous utiliserons « jeu de simulation ». La figure 1 permet de visualiser ce concept.

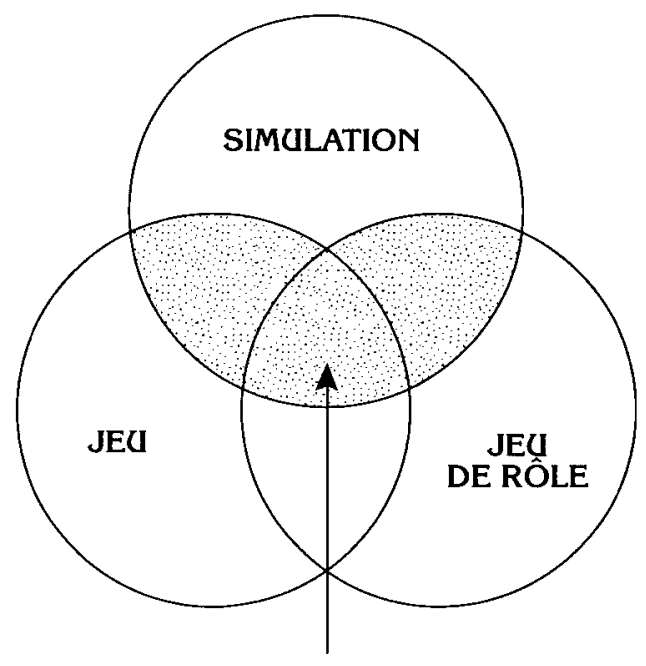

JEU DE

SIMULATION

Figure 1: Le concept de jeu de simulation

1. Le terme anglais le plus usité est simulation game traduit en français de multiples façons dont ces deux-là. 
Voyons maintenant comment nous avons bâti le présent ouvrage afin d'en faire un outil informatif et pratique pour le lecteur.

\section{Structure de l'ouvrage}

Dans le premier chapitre, nous abordons l'histoire du jeu et son émergence en tant que formule pédagogique. Dans le second chapitre, nous exposons les fondements sur lesquels reposent l'intérêt et la pertinence de ces modes d'intervention pédagogique, car, comme le dit le proverbe africain, « l'arbre ne tient pas sans racines ». Les chapitres trois, quatre et cinq portent respectivement sur le jeu, la simulation et le jeu de rôle. Chaque formule sera approfondie afin de mettre en évidence ses caractéristiques propres.

Le chapitre six porte sur la conception et la production de jeux ou simulations à vocation pédagogique. Nous y présentons un processus de développement par étapes et proposons certains remèdes à la panne d'inspiration qui bloque parfois l'élan de nombre de créateurs néophytes. Le concept de jeu-cadre est expliqué et nous discutons des avantages à exploiter ces produits facilement accessibles ou encore à les modifier à des fins pédagogiques.

Dans le septième chapitre, nous abordons les questions relatives à la préparation au jeu de rôle et à la simulation. Nous y présentons aussi l'aspect animation et les modalités d'exploitation pédagogique. Comme dans toute communication, souvent « tout est dans la façon de le faire ». Nous passons en revue les détails organisationnels à ne pas oublier et nous fournissons un cadre de référence permettant de tirer le meilleur parti possible de ces activités. 
Jeu, simulation et jeu de rôle

Enfin, au chapitre huit, nous cédons la place à quelques enseignants du primaire, du secondaire et du collégial qui ont choisi d'intégrer le jeu, la simulation ou le jeu de rôle à leur pédagogie.

Quels bénéfices en tirent-ils comme enseignants?

Quelles sont les retombées du point de vue des apprenants?

Quelles leçons ont-ils tirées de leur expérience jusqu'à présent?

Ce sont là les questions auxquelles les témoignages de ces enseignants apporteront des réponses.

En résumé, nous avons voulu, par cet ouvrage, faire un tour d'horizon des jeux, des simulations et des jeux de rôle comme formules pédagogiques.

Que sont-ils?

Que permettent-ils de faire?

Comment les utiliser?

Comment les concevoir?

Que faire pour en tirer le meilleur profit ? Voilà ce que nous vous convions à découvrir. 


\section{Chapitre 1}

\section{Survol historique}

Que l'étude, pour l'enfant, soit un jeu.

Quintilien

\section{Introduction}

L'utilisation de jeux dans le but de faire acquérir des connaissances n'est pas un phénomène récent. Un retour sur l'histoire de la plupart des civilisations nous révèle l'existence de jeux au sein des sociétés et nous informe sur leurs diverses fonctions. Malgré la perception qu'on peut en avoir, le jeu n'a probablement jamais été, dans l'histoire de l'homme, une activité gratuite. Comme l'a si bien démontré Piaget en ce qui a trait à l'enfant, le jeu s'inscrit dans le sens du développement des individus, des sociétés et des cultures. La généralité du phénomène a souvent été rapportée peu importe l'époque et les civilisations (Vial, 1981).

\section{Préhistoire}

Bien que peu de signes nous soient parvenus de la Préhistoire, nous pouvons supposer que l'homme préhistorique et ses ancêtres se sont adonnés à des activités ludiques comme il est possible de l'observer chez les animaux. Ainsi, les activités de combat, de poursuite souvent relevées chez les jeunes animaux ont une fonction de développement en vue de préparer tout leur organisme à accomplir des tâches essentielles à leur survie. L'homme préhistorique a dû lui 
Jeu, simulation et jeu de rôle

aussi, instinctivement, s'adonner à des simulacres, à des jeux que nous pourrions qualifier de « faire semblant » visant, sans doute inconsciemment, un apprentissage de gestes, un entraînement à les poser et un perfectionnement à les exécuter.

On a souvent observé dans les sociétés primitives l'existence de narrations simulées d'événements importants pour les membres de la tribu. Ces simulations permettent de conserver et d'enseigner l'histoire dans des sociétés où la tradition orale est peu développée et l'écriture inexistante.

Donc, avant même l'acquisition du langage, le jeu et la simulation ont figuré parmi les principaux instruments de transmission et d'acquisition du savoir. Le jeu du chat et de la souris et celui de la cachette, que nous avons tous pratiqués dans notre enfance, ont des origines qui remontent très loin dans notre histoire et sont un héritage commun à bien des animaux.

Les processus d'apprentissage par l'imitation ou de l'enseignement par la simulation font partie de nos plus vieilles stratégies cognitives. Dès la Préhistoire, le jeu dépasse les dimensions auxquelles, au cours de certains siècles, on tentera de le réduire, c'est-à-dire à une activité puérile à peu près vide de sens.

Les jeux reproduisent toujours la réalité d'une époque et représentent une forme d'expression de la société qui les pratique. Ils sont présents à travers tous les âges et toutes les cultures (Schwartzman, 1978). En outre, ils sont non seulement inhérents à l'humanité, mais fondamentaux dans toutes les cultures (Huizinga, 1988).

Plusieurs jeux, encore pratiqués de nos jours, remontent à des temps très anciens. Ainsi, les jeux à tablier comme les dames et les échecs ont des ancêtres millénaires. Selon des 
fresques ou dessins datant de plus de deux mille ans, ces jeux se retrouvaient sous différentes appellations et avaient des allures assez similaires. Prenons d'abord le cas du "Senet » pratiqué par les Égyptiens. Des pièces de formes semblables à celles que nous retrouvons actuellement aux échecs étaient disposées sur un damier et déplacées à partir de nombres obtenus par les dés. Le déplacement des pièces représentait l'errance de l'âme dans l'au-delà, et le hasard des dés était l'expression directe de la volonté des dieux.

Au cours de cette période et dans plusieurs civilisations, plusieurs jeux auront une fonction religieuse. Les jeux serviront à compenser l'absence de textes sacrés pour enseigner et exprimer les réalités divines.

Ainsi, l'un des plus vieux jeux qui nous soit parvenu, et qui est d'ailleurs toujours grandement utilisé, est connu sous le nom de « Mancala » ou «Wari ». Joué depuis plusieurs millénaires en Égypte (Grundfield, 1977), il est surtout répandu en Asie et en Afrique. En certains endroits, on jouait à ce jeu lors des décès. Les pleureurs s'y adonnaient pour distraire et amuser l'esprit du mort avant son départ ; l'activité devait cependant cesser au coucher du soleil, de crainte que les fantômes de la nuit ne se joignent aux joueurs et ne subtilisent leur âme.

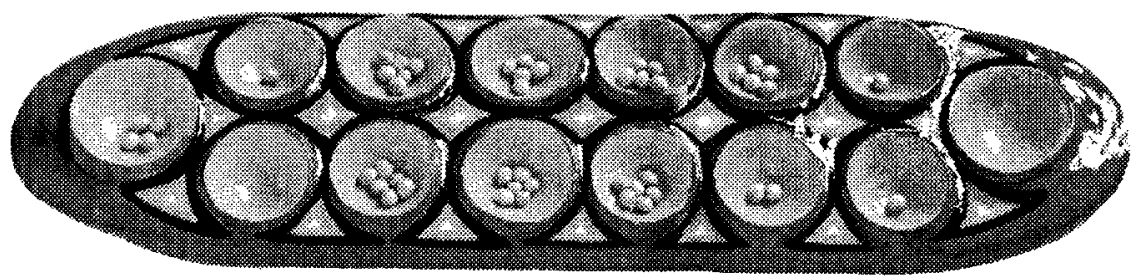

Figure 2: "Mancala" ou "Wari" 
Jeu, simulation et jeu de rôle

Bref, dans ses plus anciennes origines, le jeu a servi de représentation, de simulation, de réalités spirituelles, d'événements ou d'activités liés à la préservation tant de l'espèce que de son bagage historique et cognitif.

\section{Antiquité et Moyen Âge}

La période de l'Antiquité nous a laissé de nombreux témoignages écrits ou dessinés de l'existence et de l'utilisation de jeux au sein de la société. Les grands penseurs et les écrivains de l'époque, tels Aristote et Platon, en ont parlé. Leur vision de l'enfant et de son éducation est marquée par un certain respect de la dimension amusement qui caractérise de manière particulière cette période de la vie. Pour Platon, l'objet de l'éducation de l'enfant est d'en faire un bon citoyen. Il propose que cette éducation se fasse par l'amusement : " N'usez donc pas de violence envers les enfants, faites plutôt qu'ils s'instruisent en jouant. » (Rabecq-Maillard, 1969, p. 4)

Aristote soutient qu'il faut éviter d'imposer aux enfants des travaux et des études contraignants. Pour les préparer à leurs occupations futures, il suggère d'utiliser des jeux qui sont des imitations des activités sérieuses.

Il est intéressant de noter que les Romains donnaient à l'école le même nom qu'ils donnaient au jeu, soit ludus. Les maîtres faisaient beaucoup appel au théâtre et aux autres formes de mise en scène, qui requéraient la participation des élèves dans la représentation des fables, des légendes et de grands faits de l'histoire.

Pour enseigner l'alphabet, on avait même recours à des lettres en ivoire ou en gâteau. L'apprentissage se faisait à l'aide de jeux simples consistant à nommer les lettres, à les ordonner et probablement même, pour les gâteaux, à les manger. 
Selon Rabecq-Maillard (1969), c'est de cette époque que nous viendrait notre fameuse soupe à l'alphabet.

C'est au Moyen Âge que l'on voit apparaître le premier livre écrit sur les jeux dans la littérature européenne. Il s'agit du Libro de Juegos d'Alphonse X, roi de Castille. Ce livre, réalisé en 1283, présente une série de jeux espagnols et maures. On y traite principalement des échecs, le jeu favori du roi. Le jeu d'échecs ne date pas de cette période, mais à la faveur des croisades, on assiste à sa redécouverte. Ce jeu serait une variante du jeu indien « Chaturanga », mot sanskrit signifiant « le jeu du roi ». À l'époque, il comprenait les quatre composantes d'une armée : les éléphants, les chevaux, les chariots et les fantassins. De cet ancêtre commun se sont développés les échecs chinois et les échecs tels que nous les connaissons en Occident (Grundfield, 1977).
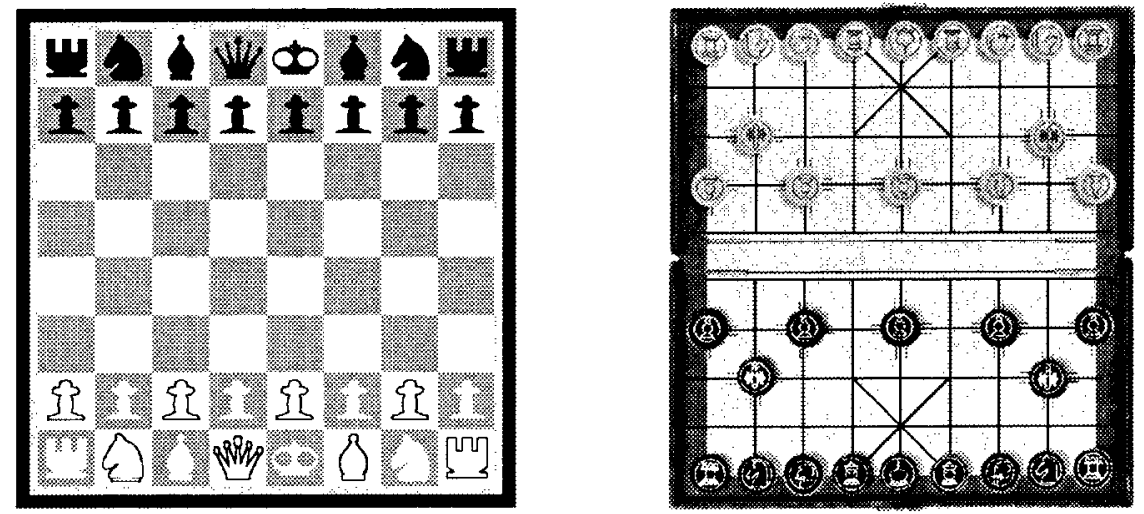

Figure 3: Les échecs et les êchecs chinois

Une légende attribue les échecs à un brahmane nommé Sissa, qui avait conçu ce jeu pour donner des leçons de morale politique à son souverain. L'intention était de montrer qu'un roi sans ses soldats était sans force et sans défense. Le roi en fut, semble-t-il, très content et offrit au brahmane 
la récompense de son choix. Celui-ci demanda au souverain de lui remettre un grain de blé pour le premier carreau de l'échiquier, deux pour le deuxième, quatre pour le troisième et ainsi de suite, jusqu'au soixante-quatrième. Le roi trouva la demande bien modeste. Le brahmane lui répondit que, tout au contraire, la possession de tous les greniers du monde ne lui suffirait pas pour remplir sa promesse. La progression arithmétique que cela implique donne un chiffre absolument faramineux. Le grenier requis pour entreposer ce nombre de grains de blé devrait mesurer 60 mètres sur 60 mètres et sa hauteur correspondre à la distance entre la Terre et la Lune.

Cette anecdote est un témoignage intéressant de l'association des mathématiques avec les jeux qui deviendra de plus en plus fréquente au cours de l'histoire.

Au Moyen Âge, le jeu d'échecs aura quelque peu évolué, s'étant adapté à l'environnement européen et aux pratiques de la guerre de cette époque. Il demeure un bel exemple d'un jeu qui témoigne de son époque par la représentation sociale qu'il véhicule.

Le jeu d'échecs que nous connaissons est en effet un modèle de la société médiévale et une simulation des guerres de l'époque. Nous y retrouvons les principaux acteurs de la société d'alors : les pions, qui représentent les paysans, ou plus précisément les serfs, sont nombreux mais sans grand pouvoir. Ils peuvent aisément être sacrifiés à des fins stratégiques. Les cavaliers correspondent aux chevaliers de l'époque au service de la noblesse ; ils représentent l'aspect offensif. Les fous, en anglais bishops (évêques), sont associés au clergé et à la politique de louvoiement qu'il devait pratiquer pour demeurer en permanence près du pouvoir. Les tours symbolisent l'aspect défensif des situations de guerre et correspondent aux bâtiments défensifs érigés un peu partout sur les territoires des royaumes au cours de cette période. Le 
roi, pièce clé du jeu, est le pivot de l'organisation sociale de l'époque. Tous ont le devoir de le protéger car, seul, il est très vulnérable. Finalement, la reine symbolise l'ensemble du pouvoir monarchique.

Le go et le jeu de serpents et échelles sont deux autres jeux qui ont longtemps servi d'outil didactique. Le go, dont l'invention remonte à trois millénaires (Bell, 1983), fut introduit au Japon au VIII ${ }^{\mathrm{e}}$ siècle, et servit pendant plusieurs siècles dans ce que l'on pourrait appeler en langage moderne les écoles militaires du Japon. Ce jeu était perçu comme propice au développement des habiletés, à la conception des stratégies guerrières tout en stimulant les fonctions intellectuelles de l'individu. Il s'agit là d'une propriété qui sera aussi attribuée au jeu d'échecs en Occident.

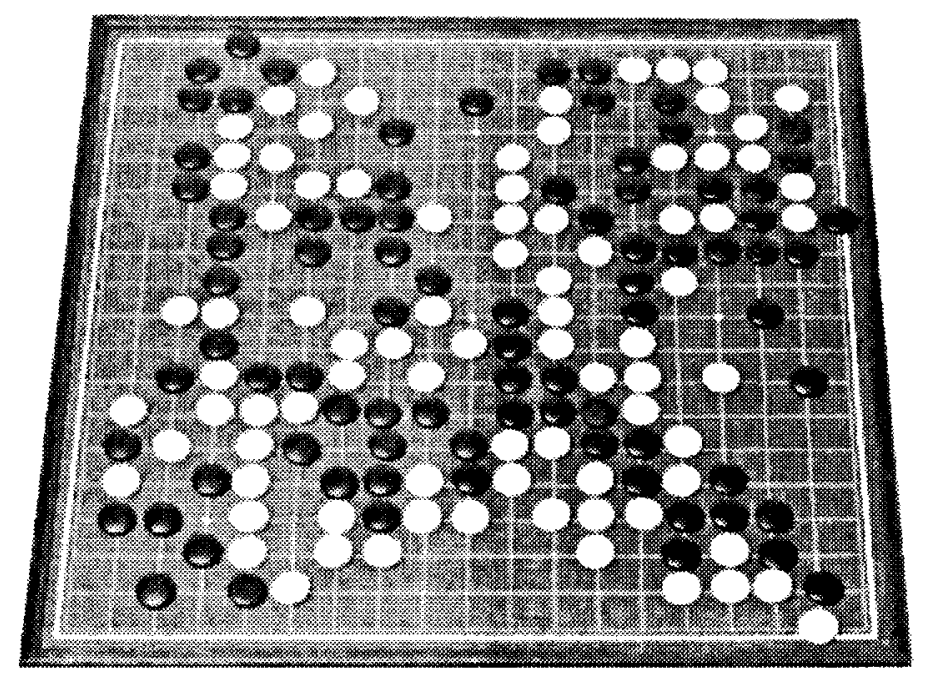

Figure 4: Le jeu de go

Le jeu de serpents et échelles nous viendrait des Indes (Bell, 1983), où il était utilisé pour l'enseignement de l'hindouisme. 
Ce jeu est une représentation symbolique du voyage de l'âme sur la terre vers le ciel. Les hindous croient en la réincarnation, une vie vertueuse permettant une réincarnation dans un être supérieur, de l'animal à l'homme, ou d'une caste inférieure à une caste supérieure. L'objectif est d'arriver, par de bonnes vies, à se libérer du cycle de la réincarnation et de parvenir à l'extase suprême, le nirvana, le ciel. Le tablier représente donc, en allant du bas vers le haut, les différents niveaux de réincarnation. Les échelles symbolisent le bien et les serpents, le mal. Une vie vertueuse permet de monter rapidement vers la perfection, donc vers le nirvana, tandis que les mauvais comportements prolongent le cycle des réincarnations, en renvoyant à des niveaux de vie inférieurs. Connu sous le nom de "Mokshapatamu », ce jeu a longtemps été utilisé pour la formation religieuse avant de nous parvenir vidé de son contenu symbolique et réduit à un simple jeu de course à obstacles.

\section{De la Renaissance au $X X^{e}$ siècle}

La Renaissance a constitué un tournant culturel important pour toute l'Europe. Pour le jeu, cette période en sera une de réhabilitation. Au Moyen Âge, période qui s'est déroulée en grande partie sous le joug d'un christianisme sévère et excessif, on associait tout ce qui était plaisir à péché. Le jeu était ainsi placé au rang des anathèmes.

La pensée humaniste, qui émerge au cours de cette période, transforme l'idée même que l'on se faisait de l'enfant et de l'enfance. Les pédagogues commencent à intégrer à l'horaire des élèves, de manière systématique, des périodes de récréation et de détente. Bien que tous ne fussent pas convaincus de l'utilité des exercices physiques, plusieurs y voyaient un facteur permettant d'améliorer le rendement intellectuel des enfants. 
Cette reconnaissance de la nécessité de simplement se récréer à l'occasion, au cours d'une journée d'école, constitue déjà un pas important vers une pédagogie moins sévère, où il n'y a pas que des exercices fastidieux et austères. Cependant, avec entre autres Érasme, le jeu s'insinuera même dans les activités d'enseignement comme telles. Selon lui, il n'y a pas de leçon plus fructueuse que celle apprise en jouant. Comme le mentionne M.-A. Stevens (1978, p. 54) : « La pédagogie humaniste se présente comme une symbiose du jeu et de l'étude : l'étude ressemble à un jeu et les lettres ne sont jamais absentes des jeux. Le colloque d'Érasme Astragalismos sine talorum lusus est le récit d'une partie d'osselets en même temps qu'une leçon d'anatomie. »

La création de la compagnie de Jésus et les collèges que les Jésuites vont instituer marquent un tournant pour la pédagogie. Le jeu n'est plus limité à l'activité physique et il envahit même le volet cognitif de la classe de l'époque. Ainsi, on tente d'exploiter au sein de la classe les stimulants auxquels l'enfant obéit pendant le jeu (Paideia, 1975). Plusieurs activités en classe seront présentées sous forme de combat. Il est proposé d'exploiter à fond l'émulation que les affrontements intellectuels permettent. À cela s'ajoutera l'utilisation de petits jeux de rôle et de pièces de théâtre, activités propices à l'enseignement et à la maîtrise du latin écrit et parlé.

$\mathrm{Au} \mathrm{XVII}^{\mathrm{e}}$ siècle, un jeu se répand partout en Europe : le jeu de l'oie. Ce jeu est fait de cases dont le nombre est très variable, généralement autour de 60, disposées en spirale et sur lesquelles les joueurs avancent selon le nombre obtenu aux dés. Ce parcours deviendra une des formes de jeu les plus exploitées à des fins éducatives. 
Jeu, simulation et jeu de rôle

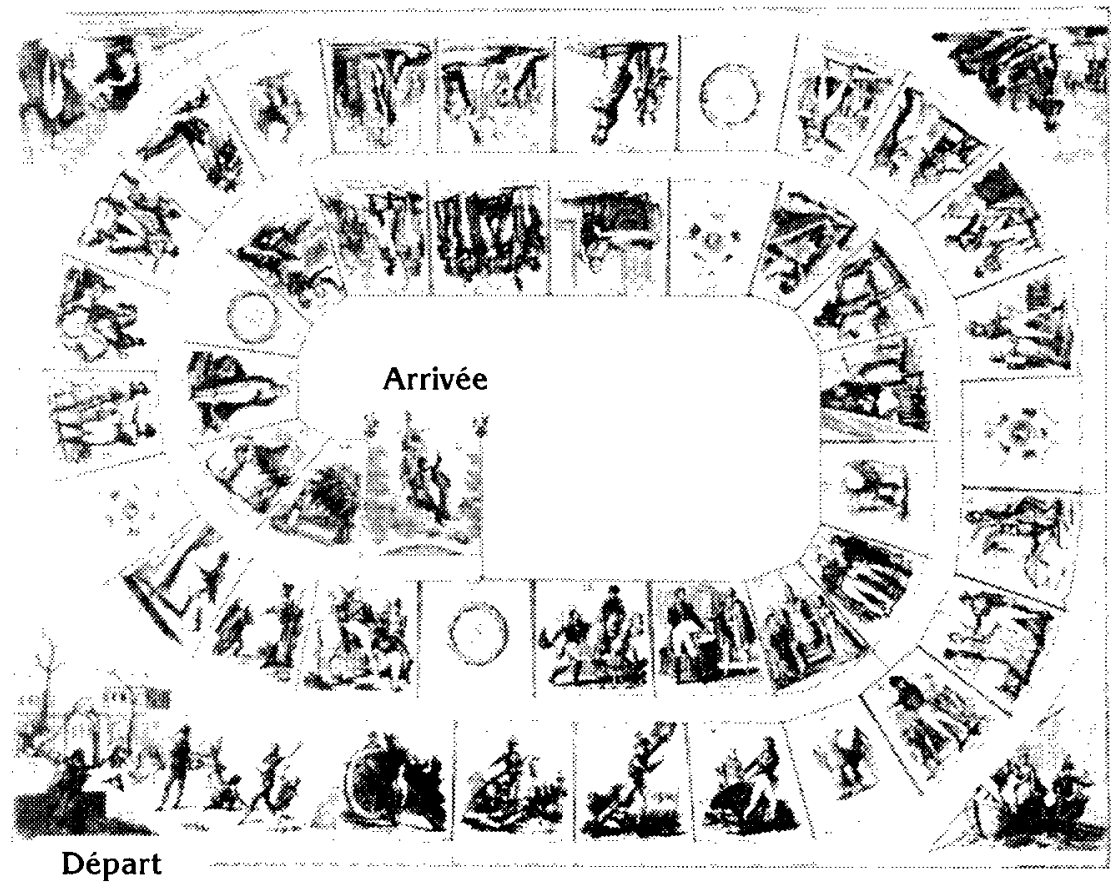

Figure 5: Structure de base du jeu de l'oie

À cette période, le jeu de l'oie donne lieu à une multitude de variantes telles que «Le nouveau jeu des vertus récompensées et des vices punis ou le triomphe de la vertu ». L'inscription sur ce jeu indique qu'il a pour objet d'instruire en amusant. Comme, en général, ils étaient élaborés à l'intention des princes, les contenus présentés porteront sur la religion, l'histoire et la science héraldique, sujets importants à l'époque.

Rousseau et Pestalozzi, ayant vécu respectivement au début et à la fin du XIX ${ }^{\mathrm{e}}$ siècle, marqueront l'époque par la nouveauté et, d'une certaine manière, par l'audace de leurs idées pédagogiques. Dans L'Émile, au siècle précédent, Rousseau promeut l'idée d'exploiter les inclinaisons de l'enfant à questionner, à agir et à explorer comme élément fondamental à 
l'apprentissage. Dans sa conception, il considère que l'adulte doit être traité en adulte et l'enfant, en enfant, et non en adulte miniature. Pestalozzi tiendra des propos similaires en soutenant que l'enfant a besoin de faire des expériences concrètes avant d'entreprendre des expériences abstraites et symboliques. C'est dans cette perspective d'ailleurs que s'inscrira plus tard toute la pédagogie de Montessori. En fait, ces auteurs proposent le respect de l'enfance. En faisant déjà entrevoir l'importance du jeu dans le développement de l'enfant, ils sont, en quelque sorte, des précurseurs de Piaget.

Tout cela aura pour conséquence l'intégration progressive dans les programmes d'études de l'époque d'activités à caractère interactif et d'exercices prenant en compte les goûts et l'environnement des enfants.

C'est aussi à cette époque que l'on assiste à un développement des kriegspiels, c'est-à-dire des jeux de guerre. Surtout populaires en Prusse, ces jeux vont rapidement se répandre en Angleterre, aux États-Unis et au Japon et serviront à la formation des officiers. Tout comme cela se fait encore aujourd'hui, ces jeux servaient à simuler des guerres afin de développer les capacités d'analyse, de résolution de problèmes et de prise de décisions des futurs officiers.

À l'aube du $\mathrm{XX}^{\mathrm{e}}$ siècle, les écoles se multiplient. Le programme scolaire typique vise à compléter les apprentissages effectués à la maison. À l'école, il faut exercer l'esprit à travers des récitations et des mémorisations, alors qu'à la maison il faut développer les muscles par les travaux physiques.

Le jeu est jugé nécessaire à la maison pour le processus d'imitation qu'il permet. Le fils observe le père, la fille observe la mère et à travers un jeu de rôle imaginaire, il ou elle reproduit l'activité du père ou de la mère. À l'école, le jeu sert à 
Jeu, simulation et jeu de rôle

libérer le surplus d'énergie physique de manière à ce qu'il n'interfère pas avec l'énergie intellectuelle nécessaire à l'étude.

Vers les années 1920-1930, l'intérêt pour le jeu va surtout porter sur ses aspects cliniques. Avec Freud, c'est tout le volet émotif qui sera étudié. Il décrit le jeu de l'enfant comme l'expression de ses désirs et de ses conflits inconscients, et il sera l'un des premiers à montrer la valeur symbolique du jeu en l'associant aux problèmes émotifs de l'enfant.

La conception même de l'école sera profondément influencée par l'industrialisation intensive qui se produit en Amérique. Les principes de productivité et de spécialisation sont les mots d'ordre ; les principes de management de Taylor seront transposés à l'école. Le jeu, considéré comme non productif, plutôt frivole, n'y aura donc pas sa place.

Cette conception de l'école sera remise en question par plusieurs pédagogues, et Dewey va proposer un retour à l'approche de Rousseau où l'accent est mis sur l'exploration, la recherche et la créativité. Comme cela s'inscrit à contre-courant de la rigidité de l'école en place, on assiste aux États-Unis à l'émergence d'écoles alternatives. L'école n'est pas là seulement pour former l'esprit et préparer l'individu à occuper une fonction dans la société : elle vise à être un élément déclencheur du changement et de l'amélioration de la société. L'inclinaison de l'enfant pour jouer doit être mise à contribution et inspirer une méthode d'enseignement des changements sociaux. L'école alternative va prôner un pluralisme, qui s'opposera au dogmatisme de l'école traditionnelle. Elle permet le plaisir et la prise d'initiatives par les élèves et, dans ce contexte, le jeu est le médium pour tout apprentissage significatif et permanent (Glickman, 1984). On propose donc à l'enfant des jeux de rôle, des jeux de socialisation et des sorties éducatives, et l'ensemble du programme est construit autour de l'enfant. 
Toute cette approche, qui demeurera cependant le fait d'une minorité, trouvera des assises solides dans les travaux de Piaget. Chez le jeune enfant, le jeu constitue une étape marquante de son développement psychomoteur et cognitif. C'est en effet par le jeu que l'enfant peut assimiler, c'est-à-dire réduire la complexité des impressions et des stimuli de son environnement à un niveau qui lui permet de les appréhender tant sur le plan intellectuel qu'affectif.

Durant la Seconde Guerre mondiale, beaucoup d'énergies sont déployées pour la formation des militaires. Les jeux et simulations figurent au nombre des stratégies de formation employées. Après la guerre, l'expérience des militaires sera transposée dans l'industrie. Le raisonnement est le suivant : si c'est bon pour la formation des militaires, ce doit l'être également pour l'industrie. De nombreux jeux d'entreprises sont ainsi mis au point. À partir de 1956, on verra même l'American Management Association se lancer sur une grande échelle dans la production et l'utilisation de jeux et de simulations destinés à former, évaluer et prévoir dans le domaine des entreprises.

Dans cette période de guerre froide de nombreux jeux de crise sont élaborés, en particulier à l'instigation de la Rand Corporation. Ils sont surtout centrés sur des simulations de crises internationales, que des joueurs, en tenant le rôle des parties en cause, tentent de résoudre à partir des points de vue qu'ils représentent.

Tous les secteurs de l'éducation et de la formation utiliseront à des degrés variés des approches qui intègrent des jeux ou des simulations. Le renouveau scolaire est plus favorable au développement global de l'individu qu'à l'apprentissage par mémorisation. 
Par ailleurs, les théories piagétiennes vont inspirer une quantité impressionnante de recherches sur les relations entre le jeu et le développement cognitif. Dans plusieurs programmes, l'utilisation du jeu sera un élément clé, particulièrement pour ceux destinés aux enfants venant des milieux défavorisés. Le Québec, avec la création des ludothèques, en est un exemple typique. La mise en place de son réseau a été réalisée dans le but de permettre aux enfants de milieux défavorisés d'avoir accès à un ensemble de jeux susceptibles de promouvoir chez eux un développement psychomoteur et cognitif comparable à celui des enfants des milieux favorisés, et ainsi diminuer les écarts de développement général souvent constatés entre les deux groupes au début du primaire.

L'utilisation du jeu comme formule pédagogique, comme outil de formation, demeure tout de même un phénomène assez généralisé en Amérique du Nord. L'esprit qui anime l'approche de plusieurs programmes scolaires visant des apprentissages significatifs par le biais de mises en situation et qui mettent en œuvre beaucoup plus que la simple sollicitation de la mémoire de l'apprenant a été propice à l'inclusion des jeux et des activités similaires parmi les stratégies employées. Il en va de même pour la formation en entreprise où la simulation et les jeux occupent une place importante dans la gamme des formules utilisées.

Ce bref survol historique nous a permis de faire ressortir le caractère évolutif des jeux et leur arrimage à la réalité de chaque époque. Qu'en est-il aujourd'hui ? Sur quoi basons-nous notre intérêt pour les jeux et simulations dans le contexte de l'enseignement que nous connaissons en ce tournant de millénaire ? Voilà une question à laquelle nous proposerons des réponses dans le chapitre deux sur les fondements pédagogiques de l'utilisation des jeux et simulations. 


\section{Chapitre 2}

\section{Fondements \\ pédagogiques}

Il me semble que tout ce qui peut être enseigné à quelqu'un est relativement peu important [...] le seul apprentissage qui influence réellement le comportement d'un individu est celui qu'il découvre lui-même et qu'il s'approprie.

Carl Rogers

Les promoteurs du jeu, et de la simulation en général, évoquent une assez grande variété de principes et des analyses fort diverses lorsqu'ils tentent d'établir les fondements pédagogiques qui soutiennent ces approches. En ajoutant aux concepts fondamentaux déjà relevés par Raser (1969), entre autres, les principaux motifs de l'intérêt pour le jeu et la simulation en enseignement peuvent être regroupés autour des trois thèmes suivants :

- une réaction à l'enseignement dit traditionnel ;

- une vision des compétences nécessaires dans le futur ;

- une conception particulière de l'enseignement.

Notons que les constats et les réflexions de cette époque sont tout à fait d'actualité plus d'un quart de siècle, et quelques virages pédagogiques, plus tard.

\section{Une réaction à l'enseignement dit traditionnel}

La plupart des systèmes éducatifs des pays industrialisés ont sporadiquement fait l'objet de vives critiques au cours des trente dernières années. Que ce soit en France, au Canada ou aux États-Unis, on doute de l'efficacité des systèmes d'éducation existants pour faire face aux exigences de la 
Jeu, simulation et jeu de rôle

société. On déplore de manière générale leur impuissance à rendre les élèves capables de maîtriser, à un niveau fonctionnel, les matières fondamentales comme la langue maternelle et les mathématiques.

Durant les années 1970, les données sur le décrochage scolaire ajoutent à l'urgence d'agir. On constate une apathie et un désintéressement de la part des élèves. Cette toile de fond plutôt désolante constitue un premier niveau d'analyse qui amène certains pédagogues à proposer une approche du processus d'enseignement qui sorte des sentiers battus. Pour secouer un système léthargique et motiver les élèves, il faut user de méthodes plus dynamiques, qui responsabilisent l'apprenant et lui font voir les liens qui existent entre les activités scolaires et le monde dans lequel il aura à vivre. C'est dans cette perspective que l'usage de jeux et de simulations dans le milieu scolaire est perçu comme pouvant contribuer à un changement d'attitude de l'apprenant (Glickman, 1979). Les mises en contexte que permettent les jeux et les simulations, la modélisation de situations pertinentes à leur vie à venir sont plus de nature à rendre signifiant l'apprentissage proposé en clarifiant ou en explicitant la relation entre l'activité à réaliser et le monde dans lequel l'élève a ou aura à vivre.

C'est paradoxalement pour les mêmes raisons que des vagues de conservatisme viendront, de manière cyclique, se manifester au sein de la société pour remettre en doute la pertinence de l'usage du jeu dans la formation, à l'école en particulier. Les tenants d'une forme d'enseignement dite traditionnelle centrée sur le manuel scolaire, les devoirs et les explications du professeur souhaitent que les enseignants fassent peu de place aux autres formes d'intervention pédagogique.

Le jeu, les simulations et les jeux de rôle refont cependant surface à la faveur des courants d'ouverture sur une pédago- 
gie plus engageante pour l'élève. C'est que, tant dans le contexte scolaire que dans celui de la formation en milieu de travail, l'atteinte de certains objectifs commande des mises en situation où il n'y a pas que la mémoire qui est sollicitée. Les jeux et simulations favorisent des apprentissages plus significatifs, puisqu'ils font appel à des stratégies de résolution de problème.

\section{Une vision des compétences nécessaires dans le futur}

Comme chacun le sait, notre société est caractérisée par le changement accéléré, par l'explosion des connaissances, dont on estime qu'elles doublent tous les dix ans. De plus, l'environnement social et technologique est en perpétuelle évolution. Les programmes scolaires, tant sur le plan des contenus que sur celui des approches et des méthodes, semblent incapables de bien préparer les jeunes au monde dans lequel ils auront à vivre.

En conséquence, l'éducation doit être définie comme un processus visant à préparer un individu à remplir des fonctions indéterminées dans des situations imprévisibles. Cette idée s'est imposée dès les années 1960.

Une des explications le plus souvent avancées pour expliquer la morosité et le désintérêt des jeunes pour l'école tient à leur perception de leurs chances de s'intégrer d'une manière satisfaisante à la société des adultes. L'accès au marché de l'emploi étant rendu très difficile, ils sont enclins à perdre espoir et à ne plus voir de sens à une préparation en vue d'occuper une situation sociale qui ne leur sera probablement jamais accessible. De plus, ceux qui se rendent jusqu'à la fin de leur cycle d'études constatent souvent que la formation 
reçue ne correspond déjà plus aux besoins du marché du travail. Comme on le relevait déjà au milieu des années 1970, l'écart entre l'environnement de l'école et l'environnement de l'apprenant en dehors de l'école ne fait que se creuser.

La question est de savoir comment préparer les élèves d'aujourd'hui à vivre dans une société que l'on ne peut véritablement prévoir. Depuis toujours, dans la plupart des sociétés, le meilleur guide pour le futur était une éducation basée sur une bonne connaissance du passé et du présent. Cela ne suffit plus. Si vous avez plus de vingtcinq ans, les mathématiques que vous avez apprises risquent fort d'être révolues et même rejetées; la biologie que vous avez étudiée n'est plus tout à fait à jour, et ainsi de suite. En supposant que vous ayez tout retenu, on peut même, jusqu'à un certain point, prétendre vous comparer à une « encyclopédie ambulante » remplie d'informations plus ou moins périmées.

Les programmes scolaires ne devraient-ils pas désormais porter l'accent sur l'acquisition d'habiletés générales ? C'est, en tout cas, le sens donné par les membres du groupe de travail sur les profils de formation au primaire et au secondaire qui insistent sur le fait que l'école doit être centrée sur les apprentissages fondamentaux (Corbo et coll. 1994). Il s'agit d'une école qui doit préparer aux rôles sociaux de la vie adulte, qui rend capable de juger et d'agir de façon responsable, une école qui éduque et fait réussir.

Pour ce faire, les jeux et la simulation, plus axés sur le développement d'habiletés générales que sur l'acquisition de connaissances particulières, constituent des modes d'intervention pédagogique à privilégier. C'est en ce sens qu'on peut affirmer qu'ils conviennent à la préparation des jeunes d'aujourd'hui à la société de demain puisqu'ils incitent à penser en termes de systèmes, de relations, de processus dynamiques plutôt qu'en termes d'événements isolés. 


\section{Une conception particulière de l'enseignement}

C'est dans la conception de l'enseignement présentée par les promoteurs de jeux et de simulation en éducation et dans la formation que l'on retrouve les idées pédagogiques les plus riches et les plus intéressantes. Ils voient l'apprenant dans une perspective très dynamique où l'autonomie, l'initiative et les processus fondamentaux de la pensée sont primordiaux. L'apprenant n'est pas un contenant qu'il faut remplir de la sagesse et du savoir du maître. La faculté d'apprendre de l'individu est plutôt perçue comme un instrument à mettre au point. L'élève est capable d'apprendre à apprendre, et c'est là que se situe l'objectif fondamental de l'éducation.

Ainsi, enseigner devient la réponse à une demande des élèves d'être stimulés avec des informations et des idées, de développer leur habileté à trouver l'information et à découvrir leurs propres idées.

On doit partir de l'apprenant et de ses intérêts pour élaborer les programmes et choisir la stratégie d'enseignement. Ainsi, le jeu sert d'abord à observer et à identifier le centre d'intérêt des apprenants. Les professeurs doivent alors utiliser ces observations pour planifier leurs interventions. Le jeu doit être supervisé et contrôlé de manière à guider intelligemment l'enfant vers des objectifs éducatifs et socialement désirables. Le goût inné de l'enfant pour le jeu doit être exploité, et le rôle de l'enseignant consiste à permettre à l'intelligence, au jugement et à l'observation d'intervenir.

Comme l'ont noté Greenblat et Duke (1975) et Saegesser (1981), le jeu et la simulation sont plutôt de l'ordre de la méthode que de la technique. Le terme technique, du grec tecknê, renvoie au travail de l'artisan qui fabrique des objets à partir d'un plan préétabli ; il reproduit des formes déjà définies. Cette notion ne peut être appliquée au jeu et à la simulation en raison 
de leur spécificité, à savoir les aspects d'aventure et d'événements inconnus et imprévisibles. Le terme méthode convient donc davantage au jeu et à la simulation. Du latin methodus qui signifie poursuite, recherche, l'expression n'exige pas nécessairement une connaissance antérieure de ce qui doit être réalisé. Elle comporte une sorte de dévoilement qui se situe au cœur même du concept de jeu et simulation. La méthode renferme la notion de devenir qui s'harmonise avec celles de créativité, d'autonomie, de gratuité associées au jeu et à la simulation.

L'apprentissage expérientiel est un autre concept clé souvent évoqué pour donner un cadre général au jeu et à la simulation dans la formation. Ce type d'apprentissage requiert l'engagement et la participation de l'apprenant. Parmi les exemples d'exercices expérientiels structurés, on retrouve de nombreux jeux et simulations. Les objectifs de cet apprentissage sont de deux ordres (Sanders et Yanouzas, 1985) :

- apprendre comment apprendre de l'expérience ;

- développer des habiletés dans les domaines des relations interpersonnelles, de la prise de décision et de la communication.

Tout ce courant pédagogique est fondé sur la prémisse que l'apprentissage est facilité quand il est combiné à une expérience concrète et que la signification est déduite de l'expérience par l'analyse. De plus, l'apprentissage semble avoir un effet accru lorsque l'expérience est accompagnée par un niveau optimal d'éveil émotionnel (Bowen, 1987).

Le modèle le plus connu et le plus cité de l'apprentissage expérientiel est celui de Kolb présenté à la figure 6 . 


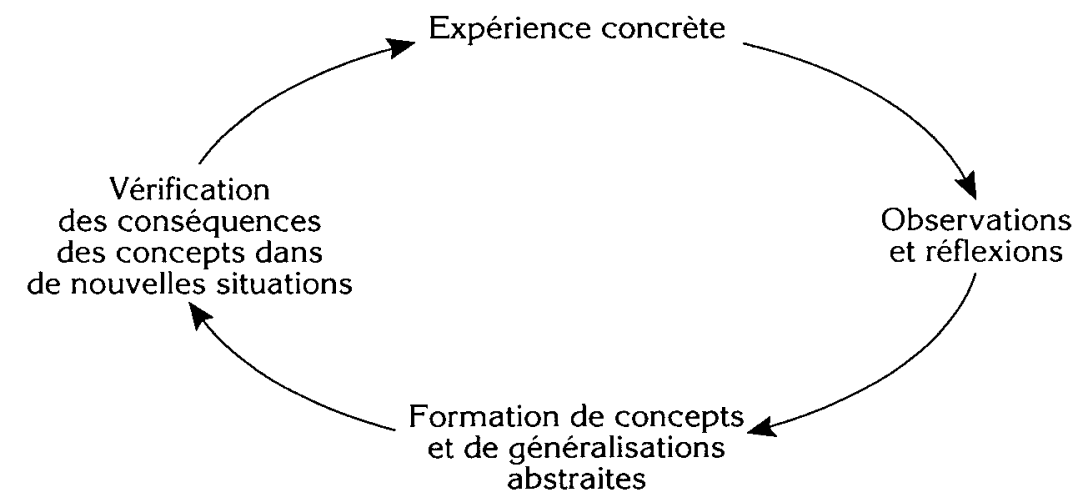

Figure 6: Modèle de l'apprentissage expérientiel de Kolb (traduit de Kolb, Rubin et Osland, 1991, p. 59)

Il s'agit donc d'un processus en quatre étapes avec itération constante. Il y a peu d'évaluation empirique sur l'efficacité de l'apprentissage expérientiel dans la production d'extrants souhaités. L'efficacité du modèle repose en bonne partie sur les capacités de l'apprenant à observer, à analyser, à réfléchir et à intégrer l'apprentissage tant cognitif qu'affectif. Le formateur y joue un rôle primordial, d'abord dans la qualité et la prégnance des exercices qu'il sélectionne et, ensuite, dans son habileté à faire réfléchir ou à généraliser sur l'expérience.

La conception de l'apprentissage ou de l'enseignement qui en émerge rejoint l'idée que l'accent en éducation doit porter sur l'engagement. La tâche est accomplie si l'on crée un environnement qui ouvre l'esprit, stimule la recherche, la curiosité, et procure des ressources pour trouver des réponses. C'est précisément l'objectif que l'on veut atteindre en utilisant le jeu et la simulation en éducation et dans la formation. L'approche atomiste, qui a longtemps prévalu dans la présentation des connaissances, cède le pas à une approche holistique où la compréhension visée tient compte à la fois de la complexité de l'environnement et des différents aspects 
Jeu, simulation et jeu de rôle

du développement de l'apprenant : cognitif, affectif et psychomoteur.

Bien des concepts seront plus facilement compris s'ils sont, pour ainsi dire, incarnés, amenés aux sens par des moyens autres que la seule manipulation des idées. C'est une pédagogie qui va du concret vers l'abstrait, du tout vers les parties; elle contraste donc avec la passivité « d'être enseigné ».

Enfin, l'option d'investir dans le jeu et la simulation dans l'éducation et la formation part aussi du principe qu'aucune formule pédagogique n'est valide pour toutes les situations et tous les types d'apprentissage (Bowen, 1987). Rien de pire qu'un professeur ou un formateur sans répertoire sur le plan des stratégies. Changer de stratégie, innover, présente certes des risques, mais il est encore plus risqué de se priver du potentiel qu'offrent différentes stratégies d'enseignement.

Reinhartz et Van Cleaf ont élaboré un continuum des stratégies pour l'enseignant, et la simulation est un élément important de cette conception du bon professeur capable de s'adapter aux situations.

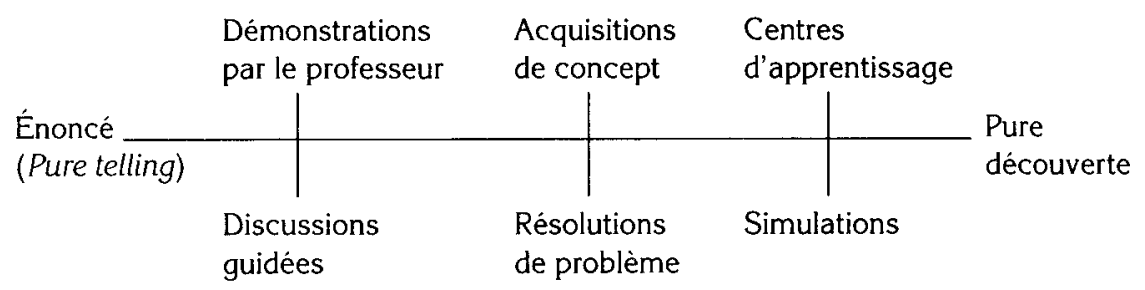

Figure 7: Continuum des stratégies de l'enseignant (traduit de Reinhartz et Van Cleaf, 1986, p. 13)

L'épigraphe de Rogers en début de chapitre peut sembler une boutade à l'endroit de ceux qui ont privilégié, dans leur pratique pédagogique, l'enseignement magistral centré sur le 
principe que l'enseignant livre le savoir et que l'apprenant le reçoit. En fait, cette proposition de Rogers rejoint jusqu'à un certain point la sagesse populaire voulant qu'on "comprenne mieux en le faisant qu'en se le faisant expliquer ».

De fait, on apprend une foule de choses sans qu'elles nous soient jamais enseignées. Nos activités quotidiennes nous placent dans des situations très variées et nous tirons, à partir de nos expériences, quantité de leçons sans le réaliser consciemment.

Cette capacité qu'a l'individu d'apprendre spontanément de ses expériences est à la base du concept d'apprentissage par la découverte ou par induction qui constitue une importante volte-face par rapport à notre conception habituelle de l'enseignement largement orientée vers une approche déductive. Afin de bien saisir jusqu'à quel point induction et déduction procèdent de manière inverse, il suffit d'examiner, au tableau 1, le parallèle proposé par Heinich, Molenda et Russell (1989).

Les situations d'apprentissage inductif sont donc particulièrement propices à la recréation du savoir telle que l'entend Artaud (1987). Ce pédagogue universitaire distingue le savoir assimilé du savoir intégré : le savoir assimilé est un savoir qui ne s'est pas arrimé à l'expérience de l'individu et qui lui est parallèle, tandis que le savoir intégré résulte d'une continuelle reconstruction du savoir d'expérience de l'individu qui s'enrichit et se diversifie à la faveur des nouveaux apprentissages.

Le jeu et a fortiori le jeu de rôle et la simulation sont des formules pédagogiques qui misent sur l'expérience de l'étudiant comme principal moment d'apprentissage. Elles permettent donc à l'étudiant de découvrir par lui-même les concepts reliés à l'objet d'étude, car il est appelé à rechercher activement l'information plutôt que d'attendre qu'elle lui parvienne. 
Jeu, simulation et jeu de rôle

Tableau 1 : Comparaison entre l'apprentissage déductif et

l'apprentissage inductif

(adapté de Heinich, Molenda et Russell, 1989, p. 311)

APPRENTISSAGE DÉDUCTIF

Présentation de l'information (l'objet d'apprentissage)

1

Application du savoir à

l'expérience de l'apprenant

En général, la stratégie déductive s'appuie largement sur le langage; son succès est donc tributaire de la compétence de l'apprenant à traiter l'information codée et symbolique. Il est possible que l'élève soit capable de bien performer lors d'un test écrit, mais qu'il soit incapable de résoudre un problème analogue dans la réalité de tous les jours.
APPRENTISSAGE INDUCTIF

Immersion dans une situation problématique réelle ou fictive

$\downarrow$

Développement d'hypothèses

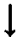

Tests d'hypothèses

$\downarrow$

Arrivée à une conclusion (l'objet d'apprentissage)

L'élève est amené à apprendre par essais et erreurs. La découverte des liens de cause à effet s'opère par les observations de l'apprenant. La compréhension du phénomène n'est donc pas asservie au décodage de l'information verbale, mais plutôt directement reliée à la réalité à l'étude.

Bien que les jeux et simulations puissent être utilisés pour un grande variété d'objectifs pédagogiques, ils sont surtout appropriés pour les objectifs d'ordre affectif ou cognitif d'un certain niveau de complexité. Cependant, la grande caractéristique des jeux et simulations est qu'ils permettent de travailler sur plusieurs objectifs simultanément et ce, dans un contexte significatif 
et intégrateur. Cette intégration constitue d'ailleurs l'apport le plus riche des jeux et simulations au monde de l'éducation et de la formation. Pris au niveau même de leur finalité, l'éducation et la formation visent le développement de toute la personne, ce à quoi l'approche holistique des jeux et simulations correspond bien.

Peu de formules pédagogiques conviennent aussi bien au domaine des objectifs affectifs. La connaissance de soi dans ses grandes dimensions et celle des autres, le développement des attitudes de tolérance et de compréhension, la capacité à entrer en communication, la prise de conscience et le développement des motivations ne sont que quelques facettes illustrant l'étendue du potentiel des jeux et simulations. 



\section{Chapitre 3}

\section{Jeu}

L'homme n'est complet que là où il joue.

Schiller

Pour la plupart des gens, le mot jeu évoque les activités dans lesquelles s'engage librement et spontanément le jeune enfant. Bien que le jeu soit l'activité privilégiée de l'enfant, il est clair qu'on ne peut le réduire à des pratiques d'activités enfantines; les jeux changent avec les âges, mais le jeu est un phénomène sans âge.

Le nombre d'expressions courantes où se retrouve le mot jeu est révélateur des multiples aspects que peut revêtir ce concept. Il suffit de consulter un dictionnaire pour constater le vaste éventail de sens attribués à ce mot. Compte tenu de la visée du présent ouvrage, la définition du jeu retenue ici est limitée au contexte des formules pédagogiques.

\section{Définition}

Interaction des apprenants dans une activité à caractère artificiel, où ils sont soumis à des règles et dirigés vers l'atteinte d'un but (Chamberland, Lavoie et Marquis, 1995, p. 65).

Cette définition ne s'appuie pas sur une fonction particulière associée au jeu, mais elle est plutôt construite autour d'attributs caractéristiques du jeu ou, si l'on préfère, de composantes essentielles : la figure 8 illustre ces quatre composantes. On appellera donc jeu une activité qui les regroupe toutes. 


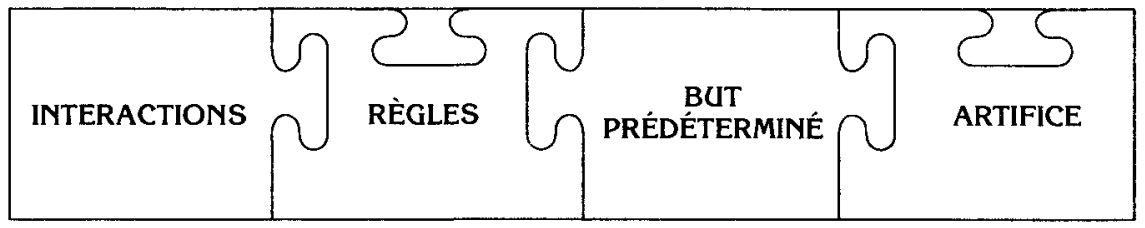

Figure 8: Les composantes d'un jeu

Le premier concept est celui d'interaction. Les interactions qui ont cours (et qui constituent l'action du jeu) peuvent prendre place tant entre deux individus qu'entre des groupes de personnes. Elles peuvent également faire intervenir des instruments, des ordinateurs, des jouets, etc., voire simplement des probabilités (par exemple, le jeu de patience). L'interaction peut également être envisagée comme le défi qu'un individu relève lorsqu'il tente de dépasser ses performances antérieures; il rivalise en quelque sorte avec luimême.

Le second attribut, ce sont les règles. Il ne peut y avoir de jeu sans règles. À partir du moment où les règles ne sont plus observées, ou bien le jeu n'existe plus ou bien nous sommes engagés dans un autre jeu. Les règles servent à orienter le cours de l'action et à déterminer la fin du jeu.

Nous faisons justement de cette règle, qui marque la fin, notre troisième attribut fondamental du jeu. En effet, il doit toujours y avoir un but clairement défini dont l'atteinte constitue la fin du jeu. Les moyens pour atteindre le but sont limités par les règles et le facteur chance intervient dans une mesure variable. Il importe d'insister ici sur le fait que le but est prédéterminé et qu'il sert à départager, le cas échéant, gagnant(s) et perdant(s).

Enfin, et c'est là son quatrième attribut, le jeu est une activité artificielle, c'est-à-dire soustraite aux normes habituelles qui s'appliquent à la réalité. Ainsi, le dénouement du jeu ne tire 
pas à conséquence dans le monde réel, ce qui vous permet de faire faillite au Monopoly sans que change votre cote de crédit auprès de l'institution financière avec laquelle vous transigez dans le monde réel!

\section{Situation typologique du jeu}

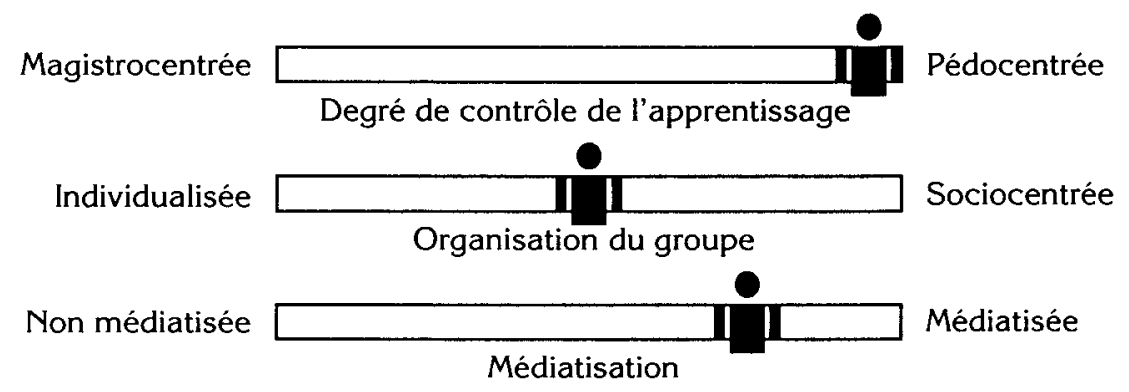

Le jeu est une formule pédagogique fortement pédocentrée puisqu'elle confère à l'apprenant une grande initiative. La présence d'autres apprenants dans le processus d'apprentissage a une importance très variable selon la nature du jeu, qui peut nécessiter plus ou moins de joueurs. En ce sens, le jeu ne peut être considéré comme une formule particulièrement individualisée ni véritablement sociocentrée. Enfin, le jeu a un caractère médiatisé, car il fait habituellement usage de matériel dont la manipulation fait partie intégrante de l'activité.

\section{Catégories de jeux}

Les jeux existent dans une variété de formes et de formats qui ont donné lieu à toutes sortes de classifications fondées sur leur envergure, le nombre de joueurs, le matériel utilisé (jeux de cartes, jeux vidéo, etc.), leur caractère physique ou 
intellectuel, leur environnement (jeu d'intérieur, jeu d'extérieur), leur fonction (jeu de société, jeu éducatif), etc. Tenant compte de notre préoccupation pédagogique, la seule classification que nous recommandons est celle du système ESAR (Garon, 1985) en raison de ses fondements psychologiques. Ainsi, nous ne nous attarderons pas à la classification des jeux afin de centrer plutôt notre propos sur une catégorie de jeux particulièrement pratique en pédagogie : le jeu à tablier.

\section{Jeu à tablier}

La forme de jeu la plus répandue, et d'ailleurs l'une des plus anciennes, est le jeu à tablier. On en retrouve chez tous les peuples sauf de rares exceptions : les Inuits et les aborigènes d'Australie (Bell, 1983). Il tire son nom du fait qu'il se joue généralement sur une table, d'où le terme tablier. Ainsi, font partie de cette catégorie tous les jeux qui se jouent sur une surface plane spécialement apprêtée à cette fin. Dans la très grande majorité des cas il s'agit d'un carton épais ou d'une planche de bois, sur lequel ont été dessinées des cases de formes carrées, triangulaires, hexagonales ou autres, servant les fins du jeu. Nous nous attarderons à cette forme de jeu en raison de la facilité avec laquelle on peut lui donner une teneur pédagogique.

\section{Caractéristiques}

Il existe un lien étroit entre les quatre composantes du jeu qui contribue à son efficacité et à l'intérêt qu'on lui porte. Au fil des ans, certaines «formules gagnantes » se sont imposées quant à la facture des jeux à tablier. Ainsi peut-on regrouper tous les jeux à tablier dans trois grandes catégories : 
la matrice à deux dimensions ;

le jeu à sentier; le modèle statique.

Notons qu'il existe des jeux qui relèvent de deux catégories à la fois : nous les appellerons les types hybrides.

\section{Matrice à deux dimensions}

Les dames, les échecs, le go et aussi le "Scrabble" sont des jeux typiques de cette

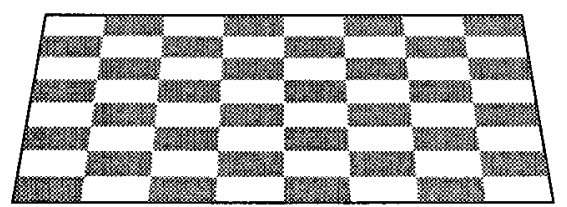
catégorie. La structure de ces jeux consiste habituellement en un tablier entièrement subdivisé selon une forme géométrique, habituellement des carrés, mais de plus en plus des triangles ou des hexagones. Les cases ainsi créées seront éventuellement occupées par les pièces selon les règles du jeu et pourront avoir des valeurs égales ou variées, tout comme les pièces utilisées. On peut cependant dire que, en règle générale, le tablier est plutôt dépouillé et les pièces parfois plus complexes que dans les autres formes de jeux à tablier (c'est le cas aux échecs).

Les règles décrivent les procédures par lesquelles les pièces sont obtenues, les directives concernant la position et le déplacement des pièces sur le tablier et la méthode pour déterminer les gagnants, soit par élimination de l'adversaire, soit par accumulation de points. Ces jeux sont caractérisés par une grande ouverture, c'est-à-dire qu'une multitude de configurations différentes sont possibles à partir d'un matériel très simple. 


\section{Jeu à sentier}

Il s'agit de jeux où l'activité principale consiste à déplacer des pièces en fonction d'un

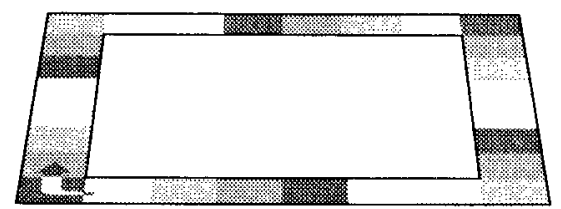
chemin à parcourir. Le mouvement des pièces n'est pas nécessairement linéaire ou circulaire. Le jeu de "Monopoly" et celui de serpents et échelles appartiennent à ce modèle de jeu à tablier.

La conception graphique du tablier détermine en grande partie la nature du mouvement des pièces. À la différence du jeu de type matrice, le tablier est généralement le support d'une foule d'informations étroitement liées au matériel accessoire (cartes, consignes écrites, dés, argent, etc.) alors que les pièces sont plutôt simples et limitées en nombre (souvent une seule par joueur).

La plupart de ces formats de jeu étant très bien connus, l'initiation à un nouveau jeu de cette catégorie se fait aisément. Ainsi, les enfants et les adolescents, lorsqu'ils sont invités, à titre d'exercice pédagogique, à créer des jeux pour présenter un contenu scolaire, pigent spontanément dans l'un ou l'autre de ces jeux à sentier.

On peut distinguer, à l'instar de Jones (1980), quatre types de jeux à sentier. 
1. Le jeu à une seule piste - Il s'agit habituellement d'une course où le gagnant est celui qui rêussit à déplacer son jeton le plus rapidement entre la case de départ et celle d'arrivée.

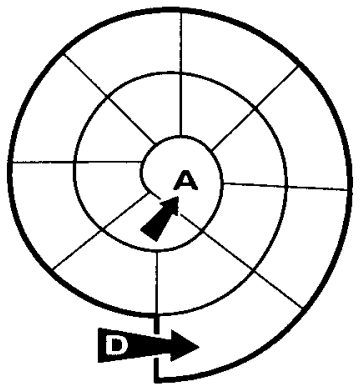

Ex. : Jeu de l'oie

3. Une piste par joueur - Chacun des participants suit un parcours donné sur lequel il est le seul à se déplacer. C'est donc une course en parallèle sans possibilité de croisement entre les joueurs sur les sentiers.

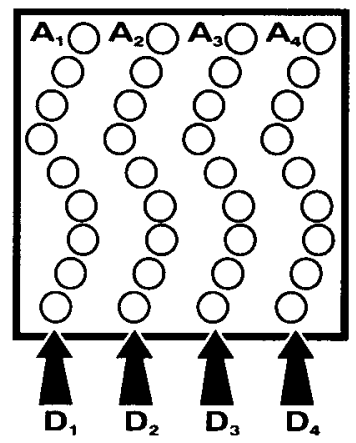

Ex.: Cribbage
2. Le jeu à une seule piste avec raccordements - Il s'agit à nouveau d'une seule piste à parcourir mais comprenant cette fois des gains ou des pertes rapides dans la distance à parcourir.

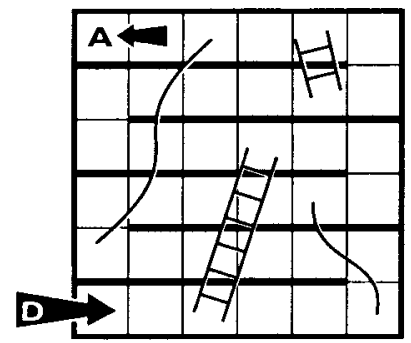

Ex.: Serpents et échelles

4. Le circuit simple - Les participants doivent le parcourir régulièrement tout au long du jeu. Il se distingue du modèle à une seule piste en ce sens qu'il n'est pas une simple course (il n'y a d'ailleurs pas de case d'arrivée).

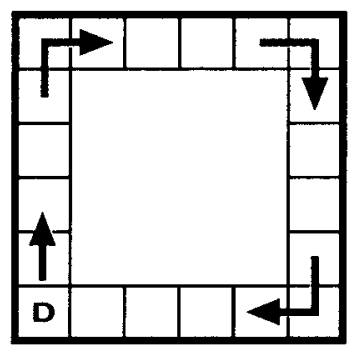

Ex. : "Monopoly"

Figure 9: Quatre types de jeux à sentier 
Jeu, simulation et jeu de rôle

Notons que, selon les objectifs pédagogiques poursuivis et la clientèle visée, l'un ou l'autre des modèles peut mieux convenir.

Le premier modèle, le jeu à une seule piste, convient à la présentation d'un nombre limité d'informations. Le jeu est donc de courte durée et ses objectifs pédagogiques sont limités au niveau de la connaissance. Le plus souvent, on retrouve sur à peu près chaque case un énoncé, une idée simple. Par exemple, dans « Le jeu de la naissance ${ }^{1} »$, qui explique le développement fotal, une case nous informe qu'à deux mois « Bébé a la taille d'une framboise».

Le deuxième modèle, à une seule piste avec raccordements, est typiquement utilisé pour véhiculer les notions de "bien » et de « mal» en termes d'actions et de conséquences. On associe un comportement souhaitable à une case d'où part une échelle en guise de renforcement positif. Inversement, la case d'où commence la glissade ou le serpent illustre un comportement indésirable. " Narcisse et Pivoine sur le chemin des floralies ${ }^{2}$ », un petit jeu éducatif pour le premier cycle du primaire, en est une illustration ; on y apprend que mettre de l'engrais favorise la croissance d'une plante mais qu'arracher l'écorce d'un arbre peut le faire mourir.

Le troisième modèle, avec une piste par joueur, convient bien aux très jeunes élèves puisqu'il limite les interactions et les conflits qui peuvent en découler.

1. Pour une description sommaire des jeux mentionnés dans les pages qui suivent, voir la section «Exemples de jeux à la fin de ce chapitre.

2. Voir la figure 10 à la page suivante. 


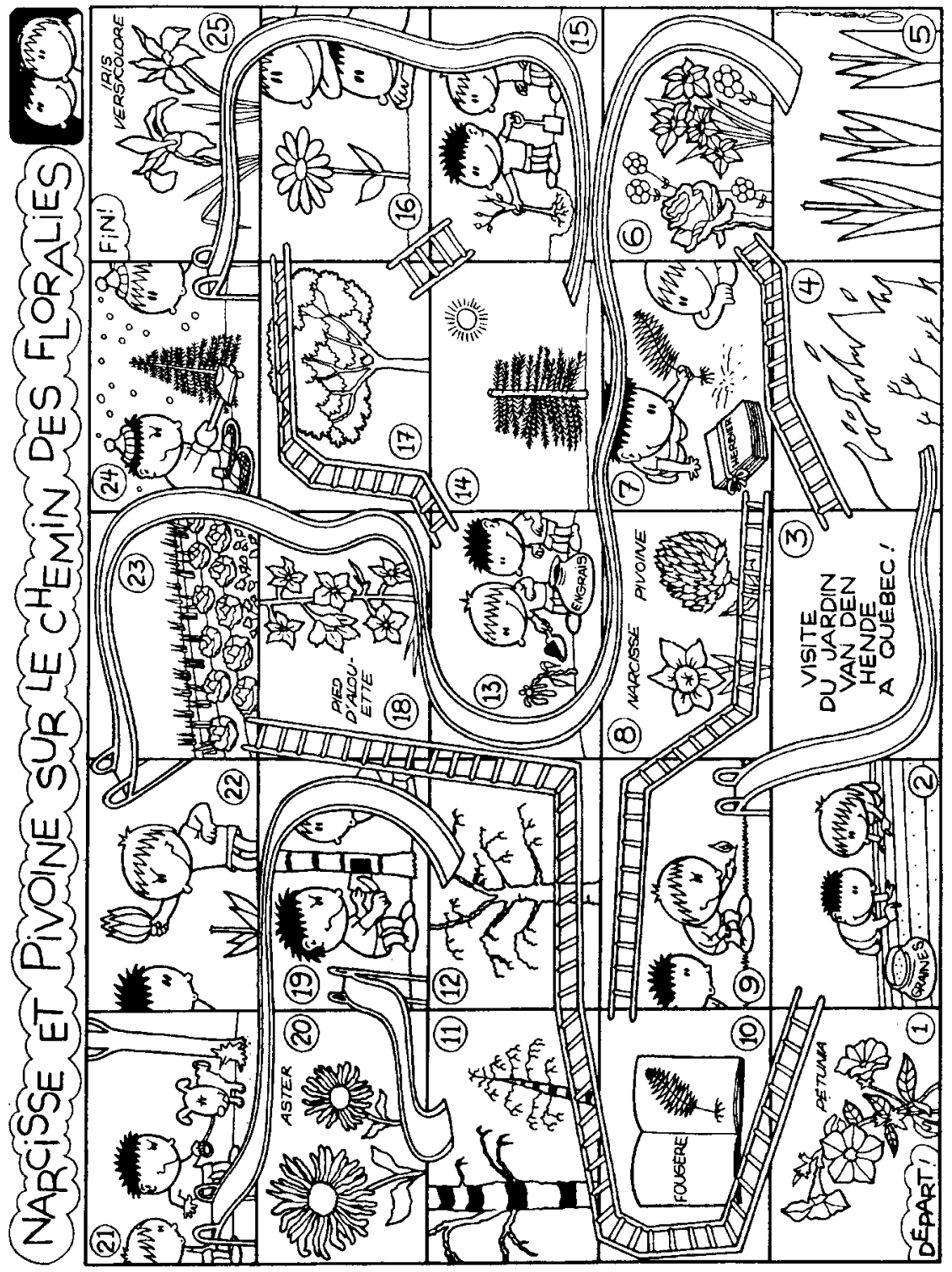

Figure 10: Exemple de sentier à une seule piste avec raccordements: "Narcisse et Pivoine sur le chemin des floralies" 
Enfin, le dernier modèle, le circuit simple, offre une grande latitude quant à la durée, au type d'interactions et à la manière dont l'information est utilisée. Les jeux de ce type comportent souvent quantité de cartes, dés, pions et autre matériel. Les cases du sentier renvoient à l'utilisation de ce matériel, et vice versa. Le « Monopoly » est bien sûr le grand classique du genre.

De manière générale, les jeux à sentier ne sont pas caractérisés par l'aspect stratégique, bien qu'il existe certaines exceptions dont Le lièvre et la tortue qui exige des joueurs une bonne dose de réflexion. Voyons maintenant le troisième type de jeu à tablier: le modèle statique.

\section{Modèle statique}

Les jeux à tablier, par leur forme et les activités qu'ils

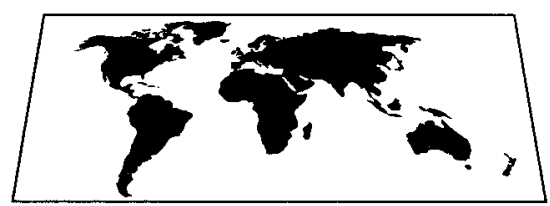
proposent, peuvent aussi

reproduire, de façon simplifiée, des réalités physiques ou reconstituer des événements. Un tablier qui illustre une carte géographique ("Risk", "Diplomacy", etc.) ou un système particulier (solaire, sanguin, etc.) constitue un modèle d'une réalité.

Le modèle statique est, en général, plus complexe que les autres types de jeux à tablier. Il a cependant un excellent potentiel pédagogique puisqu'il évoque directement la réalité abordée. Comme les joueurs visualisent tout au long de la partie l'objet d'étude qui est reproduit sur le tablier, on peut supposer qu'ils en garderont un souvenir plus clair. 


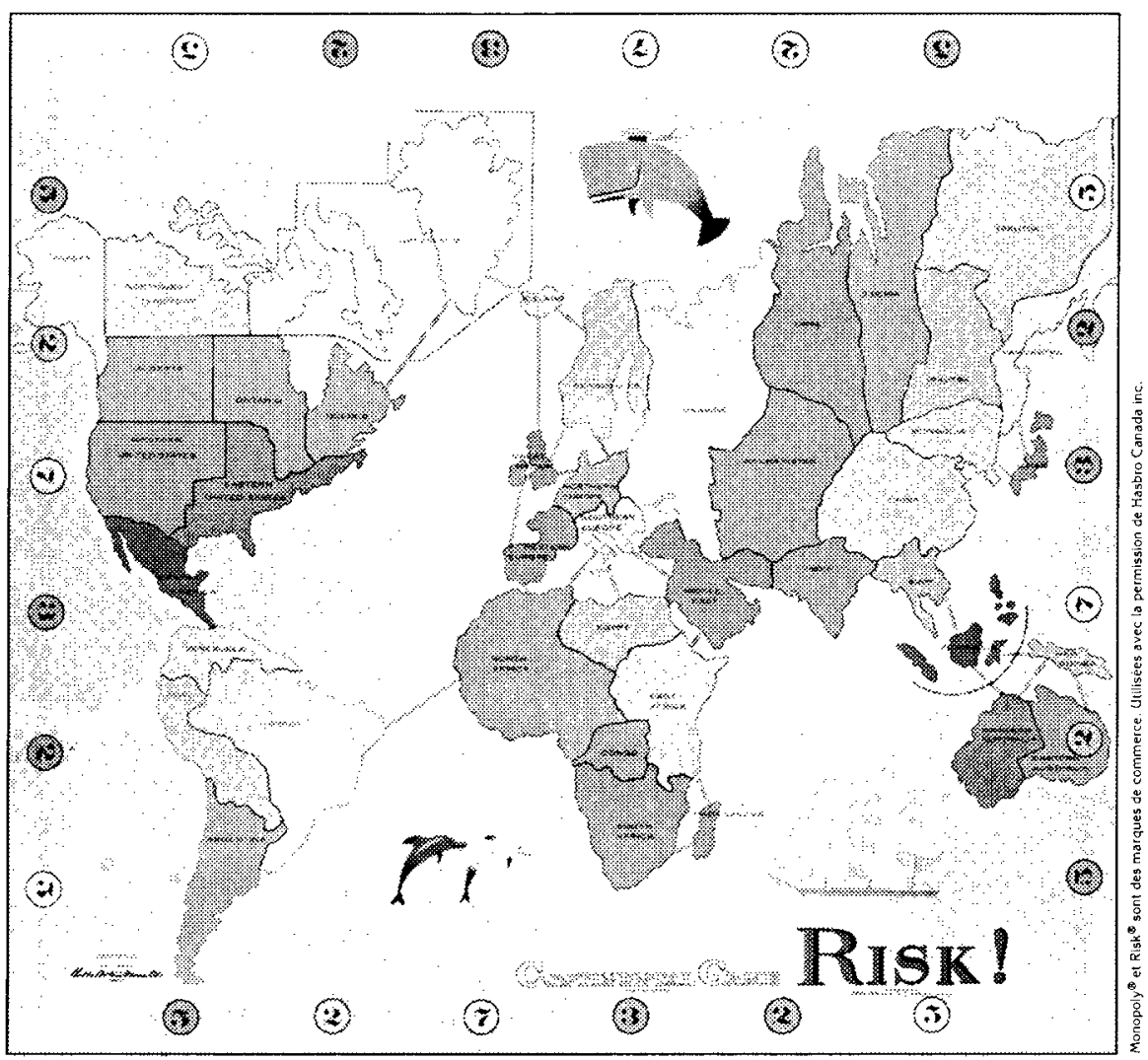

Figure 11: Exemple d'un jeu de type modèle statique: "Risk"

\section{Types hybrides}

Les trois types, la matrice à deux dimensions, le jeu à sentier et le modèle statique, ne sont pas mutuellement exclusifs. À cet égard, le jeu à tablier demeure un terrain fertile pour l'innovation. À preuve, l'excellent jeu "Labyrinthe » sort, c'est le cas de le dire, des sentiers battus. Ce jeu utilise d'ailleurs à la fois la matrice à deux dimensions et le sentier. Le sentier est en constante évolution puisqu'il est fait de cartes-cases que 
Jeu, simulation et jeu de rôle

les joueurs déplacent sur la matrice. Comme il relève de deux types à la fois, on le qualifie d'hybride.

La matrice peut aussi être jumelée au modèle statique. Enfin, un jeu à sentier peut s'apparenter à un modèle statique, comme dans « Sang bon sens » qui illustre la circulation sanguine avec un petit circuit vers les poumons et un grand circuit à travers le reste du corps.

Nous concluons cette partie du chapitre par un tableau comparant les trois types de jeux à tablier au regard de certains éléments distinctifs. 
Tableau 2: Comparaison des trois types de jeux à tablier

\begin{tabular}{|c|c|c|c|}
\hline & $\begin{array}{c}\text { Matrice } \\
2-D\end{array}$ & $\begin{array}{c}\dot{A} \\
\text { sentier }\end{array}$ & $\begin{array}{l}\text { Modèle } \\
\text { statique }\end{array}$ \\
\hline $\begin{array}{l}\text { ACTIVITÉ } \\
\text { PRINCIPALE }\end{array}$ & $\begin{array}{l}\text { Construire des patrons } \\
\text { et parfois manger les } \\
\text { pièces de l'aduersaire }\end{array}$ & \multicolumn{2}{|c|}{ Se déplacer, acquérir et échanger du matériel } \\
\hline $\begin{array}{l}\text { TABLIER } \\
\text { Design: }\end{array}$ & Dépouillé & \multicolumn{2}{|c|}{ Élaboré } \\
\hline Fonction: & $\begin{array}{c}\text { Déterminer les patrons } \\
\text { possibles et quelquefois } \\
\text { la valeur du patron }\end{array}$ & $\begin{array}{c}\text { Déterminer la nature } \\
\text { du mouvement, } \\
\text { sa direction }\end{array}$ & $\begin{array}{c}\text { Définir l'espace, } \\
\text { les aspects simulés, } \\
\text { limiter les déplacements }\end{array}$ \\
\hline Structure: & $\begin{array}{l}\text { Très polyualente, } \\
\text { très ouverte, } \\
\text { symétrique, } \\
\text { régulière }\end{array}$ & $\begin{array}{l}\text { Très limitative, } \\
\text { figée, fermée, } \\
\text { semi-symêtrique, } \\
\text { semi-régulière }\end{array}$ & $\begin{array}{c}\text { En relation étroite avec } \\
\text { la réalité reproduite par } \\
\text { le modèle, asymétrique, } \\
\text { irrégulière, parfois } \\
\text { deux superposées }\end{array}$ \\
\hline
\end{tabular}

\begin{tabular}{|c|c|c|c|}
\hline \multicolumn{4}{|l|}{ PIĖCES } \\
\hline Nombre: & $\begin{array}{l}\text { Élevé, plusieurs } \\
\text { par joueur }\end{array}$ & $\begin{array}{l}\text { Faible, souvent } \\
\text { une par joueur }\end{array}$ & Variable \\
\hline Valeurs: & $\begin{array}{l}\text { Différentes selon } \\
\text { leur nature et } \\
\text { parfois variables }\end{array}$ & Toutes égales & Variables \\
\hline $\begin{array}{l}\text { MATÉRIEL } \\
\text { DE SOUTIEN }\end{array}$ & $\begin{array}{l}\text { La plupart du temps } \\
\text { inexistant }\end{array}$ & \multicolumn{2}{|c|}{$\begin{array}{c}\text { Élaboré, sujet à achats, échanges } \\
\text { et en étroite relation avec le tablier, } \\
\text { l'un renvoyant à l'autre }\end{array}$} \\
\hline \multirow[t]{2}{*}{ RÈGLES } & \multirow{2}{*}{$\begin{array}{c}\text { Simples, doivent être } \\
\text { connues à l'avance } \\
\text { du joueur }\end{array}$} & \multicolumn{2}{|c|}{$\begin{array}{l}\text { De complexitè variable, concernent } \\
\text { surtout le déplacement des pièces }\end{array}$} \\
\hline & & $\begin{array}{l}\text { Quelques règles de base } \\
\text { puis consignes sur } \\
\text { le tablier ou sur } \\
\text { le materiel de soutien }\end{array}$ & $\begin{array}{l}\text { Relation des pièces } \\
\text { entre elles }\end{array}$ \\
\hline $\begin{array}{l}\text { PLACE } \\
\text { DU HASARD }\end{array}$ & Faible & Élevée & $\begin{array}{c}\text { Variable, habituellement } \\
\text { moyenne }\end{array}$ \\
\hline
\end{tabular}


Jeu, simulation et jeu de rôle

\section{Avantages}

L'avantage le plus spontanément évoqué par les enseignants qui utilisent le jeu comme formule pédagogique est le grand intérêt qu'il suscite chez les élèves de tout âge. Le jeu est motivant.

Dans la foulée de ce qui a été expliqué dans le chapitre sur les fondements pédagogiques, notons que le jeu donne beaucoup de place à l'élève dans le processus enseignement-apprentissage. Bien souvent, le jeu peut être géré par les élèves eux-mêmes, sans animateur. Le jeu favorise l'autonomie de l'élève.

La majorité des jeux nécessite une interaction entre plusieurs élèves et les place en situation de ressentir et d'exprimer leurs joies et leurs frustrations avec leurs pairs. De plus, il favorise le sentiment d'appartenance au groupe. Le jeu est socialisant.

La plupart des jeux sont complets en eux-mêmes. Leur utilisation ne nécessite pas d'autres éléments que ceux compris dans les boîtes, ce qui permet de les ranger facilement. Ils s'utilisent dans un espace restreint; pas besoin d'autres locaux. Le jeu est pratique.

Comme le hasard a une certaine importance dans l'issue du jeu, même les moins doués d'un groupe, ceux qui ont peu d'occasions de se faire valoir dans un contexte plus scolaire, peuvent ressortir vainqueurs et connaître leur heure de gloire Le jeu est valorisant.

On peut intégrer au jeu des notions et des informations qui font habituellement l'objet d'exercices répétitifs. En ce sens, le jeu est le contenant qui fait bien passer le contenu. On 
l'utilise ainsi souvent comme complément d'une autre formule en guise de révision ou de synthèse de ce qui a été vu. Le jeu favorise le renforcement.

\section{Limites}

La gamme de jeux disponible sur le marché pouvant favoriser l'atteinte d'objectifs pédagogiques précis est très limitée, surtout en langue française. Il existe peu de jeux utilisables tels quels.

Par ailleurs, l'enseignant intéressé à développer ses propres jeux aura besoin de temps, d'argent et d'une certaine habileté pour produire un jeu attrayant et durable. De plus, la nécessité de pouvoir disposer de plusieurs exemplaires pose parfois des problèmes. Produire ses propres jeux est exigeant ${ }^{2}$.

Son potentiel pédagogique demeure limité à des objectifs simples, surtout en ce qui a trait à la connaissance et à la compréhension. Le jeu ne convient pas à tous les types d'objectifs.

Il faut que le contenu éducatif soit bien intégré dans le processus d'enseignement, sans quoi l'activité peut facilement devenir un jeu pour un jeu dont on tire peu de bénéfices sur le plan des apprentissages. Il doit y avoir un certain équilibre entre le hasard et l'habileté des joueurs comme facteur de réussite dans le jeu. Il y a risque de passer à côté du contenu éducatif.

Les apprentissages réalisés durant le jeu ne sont pas automatiquement transférés à d'autres contextes ; cela varie d'un

\footnotetext{
2. Cette limite peut cependant être contournée par le recours au jeu-cadre. À cet égard, voir le chapitre 6
} sur la conception des jeux, simulations et jeux de rôle. 
apprenant à l'autre. Ainsi, certains apprenants ont besoin d'une méthode complémentaire pour intégrer pleinement le contenu éducatif. Le transfert des apprentissages n'est pas assuré.

L'excitation qu'il engendre, les conflits qu'il peut susciter, le niveau de bruit qui peut résulter sont autant de facteurs qui obligent l'enseignant à bien définir ses attentes à l'égard des comportements des joueurs. Le jeu exige un encadrement disciplinaire.

\section{Conditions d'utilisation}

Certaines conditions doivent être réunies lorsqu'on utilise des jeux afin d'assurer leur contribution au processus d'apprentissage.

Quand on a recours à des jeux déjà disponibles (commerciaux ou autres), la première condition d'efficacité est un choix judicieux. Les champs d'intérêt et les aptitudes des apprenants doivent être considérés au même titre que le potentiel pédagogique. Les jeux doivent être soigneusement sélectionnés.

Lorsqu'on produit ses propres jeux, on doit éviter de perdre de vue les objectifs pédagogiques au profit du caractère ludique. Le type de jeu doit correspondre aux compétences que l'on souhaite faire acquérir. La validation du jeu par des collègues de travail et par des apprenants qui fourniront leur appréciation d'une version préliminaire en garantira l'efficacité. La création de jeux suppose une démarche rigoureuse. 
Il convient de compléter les interventions pédagogiques sous forme de jeux par d'autres formules en guise de renforcement. Que le jeu précède ou suive l'autre type d'intervention, le transfert des compétences sera augmenté. On gagne à jumeler le jeu avec d'autres formules.

L'enseignant qui crée ses propres jeux serait bien avisé de recourir à des formes de jeu connues ou alors très simples. Les apprenants ont ainsi moins d'efforts à faire pour comprendre les règles et ils peuvent mieux se concentrer sur le contenu. Les jeux simples ou familiers à l'apprenant sont plus efficaces.

Cette dernière idée est l'un des fondements du concept de « jeucadre » que nous présenterons au chapitre 6 . 


\section{Exemples de jeux}

\section{«'Smath »}

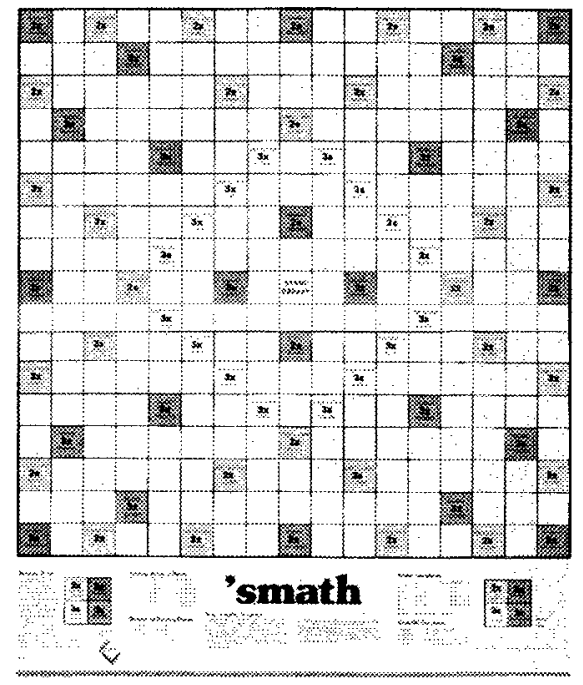

(distribué au Canada par Playtoy)

Type : Jeu à tablier avec matrice à deux dimensions

Clientèle : Deuxième cycle du primaire et secondaire

Nombre de joueurs : 2 à 4

Durée : 60 à 90 minutes

Coût : Environ $15 \$$

Ce jeu peut être utilisé pour renforcer la capacité de calcul mental. C'est le pendant arithmétique du « Scrabble ». La ressemblance entre les deux jeux est d'ailleurs frappante, tant sur le plan de la présentation matérielle que sur celui du principe de fonctionnement. Les joueurs effectuent des opérations d'addition, de soustraction, de multiplication et de division sur le tablier et accumulent des points en fonction des résultats de ces opérations. Certaines cases restreignent le choix des opérations alors que d'autres sont primées et permettent de doubler ou tripler les points obtenus. Comme pour le « Scrabble », le jeu se termine après épuisement des jetons. 


\section{« Le jeu de la naissance »}

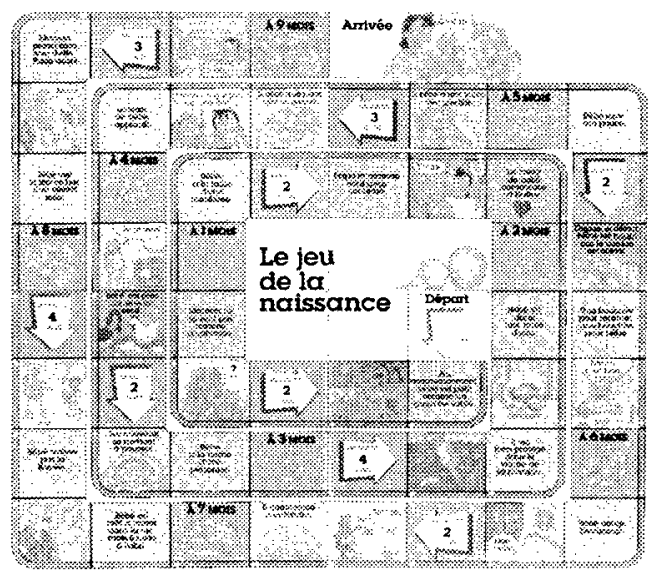

par Marie-France Hébert (le jeu est inclus dans le livre Venir au monde, Éditions La courte échelle)

Type : Jeu à tablier avec un sentier en circuit simple Clientèle : Préscolaire et premier cycle du primaire Nombre de joueurs : 2 à 4 Durée : 15 minutes

Coût : Environ $20 \$$

Ce jeu vise à faire connaître aux enfants les diverses étapes de l'évolution du bébé dans le ventre de sa mère. La case de départ correspond à la fécondation et la case d'arrivée, à la naissance. Les cases du circuit en spirale illustrent certaines étapes de l'évolution $\mathrm{du}$ foetus et donnent un aperçu de la vie quotidienne des parents durant la grossesse. D'autres cases comportent des consignes pour faire avancer plus vite ou reculer de quelques cases. On y avance à l'aide d'un dé. 


\section{« Le lièvre et la tortue "}

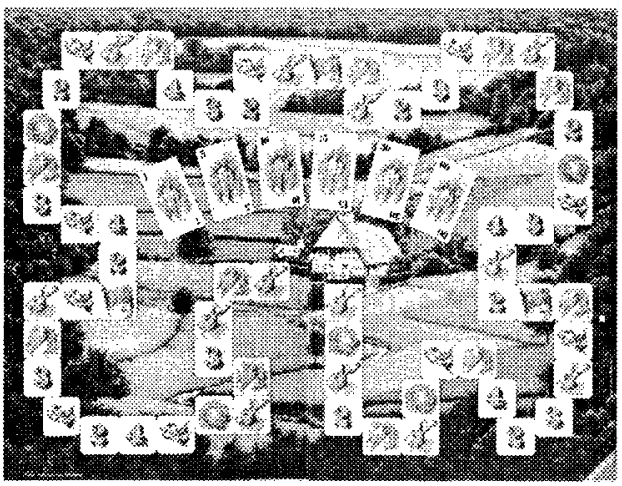

(jeu Ravensburger qui n'est plus vendu au Canada) Type : Jeu à tablier avec un sentier en circuit simple Clientèle : Deuxième cycle du primaire et secondaire

Nombre de joueurs : 2 à 6 (de préférence 4 ou plus) Durée : 60 à 90 minutes Coût: Environ $50 \$$

Ce jeu peut servir à développer la capacité des élèves à formuler des hypothèses et à retenir la stratégie ayant la plus grande chance de succès. Il s'agit simplement d'une course sur un parcours unique. À chaque tour, les joueurs avancent du nombre de cases qu'ils désirent et doivent payer ce déplacement avec des carottes. Le nombre de carottes exigé croît en fonction du nombre de cases franchies selon une progression géométrique ( 1 case $=1$ carotte, 2 cases $=3$ carottes, ..., 10 cases $=55$ carottes, ..., 20 cases $=210$ carottes, etc.). Il faut donc doser sa progression. Le joueur qui prédit correctement le rang qu'il occupera au moment de rejouer reçoit un boni, toujours en carottes. On peut reculer sur le parcours pour en accumuler d'autres. Il faut obligatoirement passer quelques tours sur place, histoire de manger les laitues» dont il faut se départir avant l'arrivée. Il faut d'ailleurs arriver au but « léger », donc « dépenser » presque toutes ses carottes sur le parcours.

On peut donc adopter une progression régulière (style « tortue ») ou démarrer très lentement et accumuler beaucoup de carottes pour coiffer l'adversaire au fil d'arrivée dans un sprint spectaculaire (style " lièvre »). Mais le vainqueur sera celui qui analysera le mieux la stratégie de ses adversaires. 


\section{« Labyrinthe » (et « Labyrinthe Master »)}

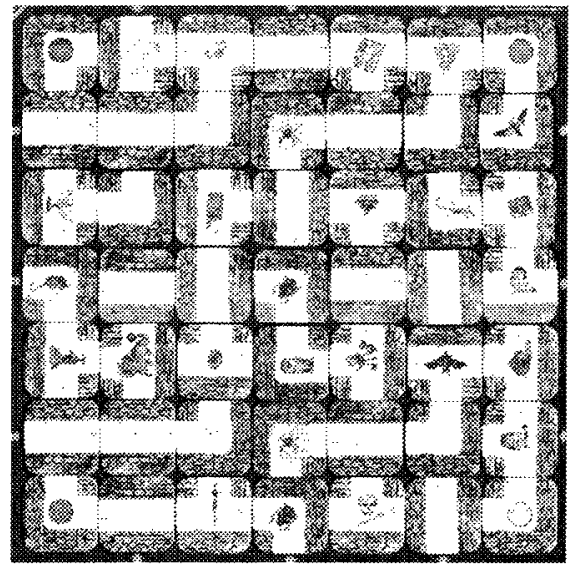

(jeu Ravensburger)

Type: Jeu à tablier de type hybride: superposition de sentier et de matrice à deux dimensions

Clientèle : Deuxième cycle du primaire et secondaire

Nombre de joueurs : 2 à 4

Durée : 30 à 60 minutes

Coût : Environ $40 \$$

Labyrinthe est un autre jeu qui comporte la formulation d'hypothèses et la planification de stratégies. Son originalité lui vient du fait que le sentier évolue constamment, à mesure que chacun joue son tour. En effet, le réseau de sentiers est fait de cartes qu'on manipule en cours de partie. En déplaçant les cartessentiers, on ouvre de nouveaux passages tout en en fermant d'autres dans le labyrinthe. Les joueurs rivalisent de stratégies pour que le labyrinthe les avantage dans leur progression et nuise à celle des adversaires. Le but du jeu est de cueillir, dans un ordre défini, certains objets dispersés sur les sentiers. Si l'on demandait aux élèves d'écrire ou de verbaliser leur stratégie pour chaque tour, le jeu pourrait servir de prétexte à des exercices de syntaxe et de con jugaison (notamment du conditionnel), soit en langue maternelle ou dans une langue seconde. 


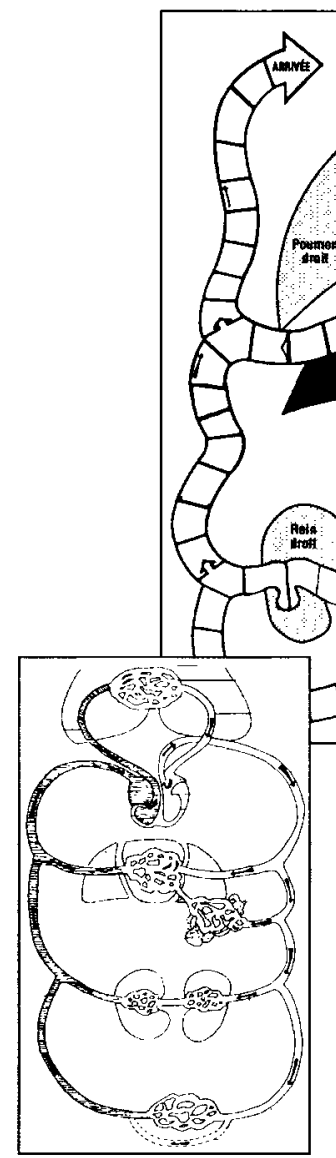

(adaptation de The Great Blood Race, auteur inconnu; non disponible) Type : Jeu à tablier intégrant un sentier et un modèle statique

Clientèle : Secondaire

Nombre de joueurs : 2 à 4

Durée : 30 à 45 minutes

Ce jeu a pour objet la compréhension du système circulatoire sanguin. Le sentier suit le réseau d'artères, de veines et de capillaires pour atteindre tour à tour les organes du corps humain (cœur, poumons, foie, reins, cerveau, membres). Au fil du parcours, le joueur pige des cartes, tantôt informatives, tantôt prescriptives (qui lui dictent une action). On distingue clairement la circulation vers les poumons (pour recueillir l'oxygène) de celle vers les organes (pour nourrir les tissus). 


\section{« Diplomacy »}

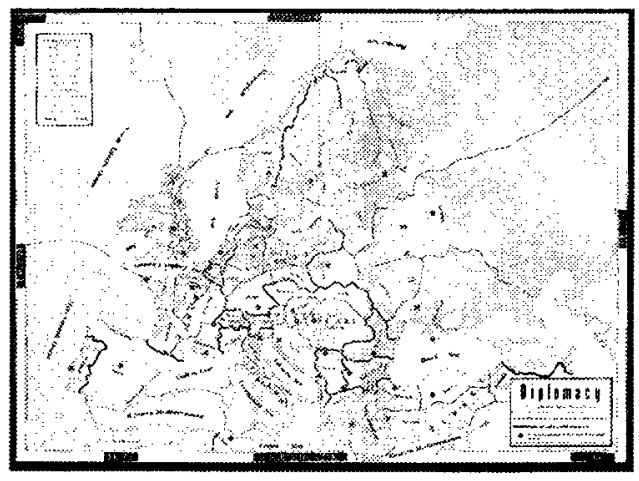

(jeu Monarch Printing)

Type: Jeu à tablier de type modèle statique intégrant une part de simulation et de jeu de rôle

Clientèle : Deuxième cycle du secondaire et collégial

Nombre de joueurs : 2 à 7 (de préférence 5 ou plus)

Durée : 3 heures et plus

Coût : Environ $75 \$$

Ce jeu peut être utilisé pour illustrer la complexité des alliances stratégiques et des relations diplomatiques entre États. Il peut servir à l'enseignement de principes généraux de négociation. Le jeu se déroule par cycles de tractations entre les joueurs (qui s'isolent deux par deux) puis de manipulation du matériel sur le tablier qui représente une carte simplifiée de l'Europe. Les manipulations reflètent les décisions des joueurs d'attaquer, de se défendre ou de soutenir une attaque d'un tiers. Des pertes et des gains sont ainsi enregistrés et conduisent les joueurs à chercher de nouveaux appuis lors de la période de tractations subséquentes. Pour gagner, les joueurs n'ont d'autres choix que de rompre éventuellement certaines alliances. 



\section{Chapitre 4}

\section{Simulation}

J'entends et j'oublie ;

Je vois et je me souviens;

Je fais et je comprends.

Confucius

\section{Définition}

Reproduction d'une situation constituant un modèle simplifié mais juste d'une réalité (Chamberland, Lavoie et Marquis, 1995, p. 81).

La simulation n'existe que par analogie à la réalité. C'est pourquoi on la qualifie de reproduction ou modèle d'une réalité. Insistons sur le fait que, bien que simplifié, le modèle doit être juste puisque la fonction essentielle de la simulation est de permettre une meilleure compréhension de la réalité. De plus, le facteur temps intervient généralement dans ce modèle, ce qui lui confère un caractère dynamique.

Simuler, c'est donc modéliser la réalité. La réalité est plus facile à comprendre lorsqu'on la dépouille de certains éléments qui la rendent complexe et qu'on ne fait ressortir que certains aspects jugés importants. L'exercice en est donc un de simplification pour faciliter la description, l'analyse ou la compréhension des faits, des lois et des comportements de tout ce qui constitue notre monde (Greenblat, 1988). 


\section{Situation typologique de la simulation}

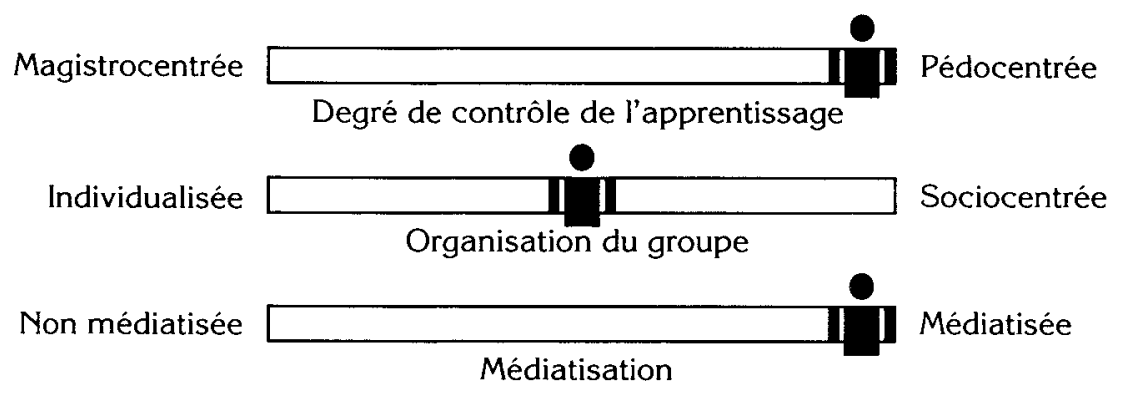

La simulation est résolument pédocentrée, car elle place l'élève aux commandes de son apprentissage. En effet, la simulation favorise mieux que d'autres formules pédagogiques un type d'apprentissage résultant de manière naturelle des expériences courantes de l'apprenant. Cependant, à la différence de l'expérience journalière de l'individu, elle est basée sur des situations factices et sur des activités de réflexion complémentaires.

Il n'y a pas de tendance marquée quant à la dimension de l'organisation du groupe ; cette formule n'est donc ni individualisée, ni sociocentrée. Enfin, son caractère médiatisé est systématique.

\section{Caractéristiques}

Pour bien saisir le concept de simulation, il faut préciser ce que l'on entend par " modèle » de la réalité. On peut considérer deux catégories de modèles : les modèles statiques et les modèles dynamiques.

Les modèles statiques représentent des objets ou des situations sans processus de variation. Une carte géographique en est un exemple : elle représente une réalité physique à un 
moment déterminé. Par contre, les modèles dynamiques, dans leur représentation d'une réalité, incluent des variables de modification, de mouvement, de développement, d'évolution dans le temps. La maquette mise au point pour étudier l'effet de la crue des eaux sur un barrage ou le Parlement modèle auquel participent des élèves du secondaire sont des modèles dynamiques. Généralement, seuls les modèles dynamiques seront appelés simulations (Becker et Gouddappel, 1972).

Certains auteurs ont proposé un autre genre de distinction: d'une part, les modèles micromorphes et, d'autre part, les modèles paramorphes (Rickey, 1986). Les modèles micromorphes correspondent aux représentations physiques, aux répliques à une échelle inférieure des réalités qui s'y prêtent. Les modèles paramorphes sont associés aux représentations « intellectuelles », qui font référence à la réalité par l'intermédiaire d'un code symbolique, habituellement le verbal. C'est donc de modèles paramorphes qu'il est plus question en simulation.

Selon ces modèles et dans un contexte d'éducation et de formation, McLean et Raymond (1976) distinguent trois grandes catégories de simulations :

- la simulation/personne ;

- la simulation/machine ;

- la simulation personne/machine.

La simulation/personne (que nous désignerons par « simulation humaine ») n'implique que des personnes dans son déroulement. Surtout utilisée pour l'enseignement, elle est constituée de modèles de réalités sociales dans lesquelles interagissent des personnes. Une méthode de formation comme le micro-enseignement ou la simulation "They Shoot Marbles, Don't They ? " en sont d'excellents exemples. 
La simulation/machine comporte l'utilisation de l'ordinateur dans un contexte de recherche. À partir de modèles habituellement mathématiques, on utilise l'ordinateur pour étudier l'effet de différentes variables sur le système simulé. Ainsi, une simulation de ce type ayant pour objet la croissance de la population permettra d'étudier les effets de l'augmentation en nombre d'un groupe sur les ressources alimentaires disponibles ou sur la consommation d'énergie. L'ordinateur est donc l'élément principal de ce type de simulation.

La simulation personne/machine (nous utiliserons le terme «simulation informatique ») relève des deux types précédents. L'ordinateur est utilisé comme instrument d'assistance à la prise de décision. On lui fait effectuer des calculs, on consulte les banques d'information qu'il contient. Les systèmes experts sont au service des personnes qui orientent le déroulement de la simulation. C'est un type fréquemment utilisé dans les sciences de l'administration.

\section{Simulateurs}

Dans le domaine des apprentissages moteurs, on emploie les simulateurs qui sont, à la simulation, ce que le jouet est au jeu. Les simulateurs sont des appareils qui permettent la pratique d'activités motrices dans un contexte de rétroaction immédiate. Le simulateur de vol, avec ses cadrans, ses manettes, boutons et autres instruments de contrôle offre un environnement assez semblable à celui retrouvé dans un véritable cockpit d'avion pour qu'on puisse y apprendre les manœuvres de pilotage et ce, grâce aux réactions simulées et instantanées de l'appareil aux actions que pose l'apprenti pilote.

On imagine facilement les avantages qu'il y a de pouvoir s'exercer à des tâches complexes comme le pilotage d'un 
avion sans avoir à subir les conséquences d'erreurs de manipulation qui seraient fatales dans la réalité. Outre l'avantage évident au regard de la sécurité, les simulateurs permettent des économies appréciables de temps et d'argent (par exemple, le temps des contrôleurs aériens, les coûts d'entretien des appareils et de l'essence, etc.).

Le facteur temps pose souvent des problèmes pour l'étude des phénomènes extrêmement lents (pensons au domaine de l'astrophysique) ou extrêmement rapides (comme en électronique).

La simulation permet de contourner d'autres types de difficultés, que ce soit d'ordre déontologique (par exemple, au cours de recherches sur la violence on ne peut, sur le plan éthique, être violent envers les sujets) ou d'ordre logistique (comme l'impossibilité de réunir les conditions nécessaires au moment prévu pour l'étude du phénomène).

\section{Validité}

Pour tirer quelque bénéfice de la simulation, il faut qu'elle soit valide, c'est-à-dire qu'elle ne fausse pas la réalité qu'elle modélise. Toute la question de la validité d'une simulation repose sur sa fidélité à représenter la réalité. Pour augmenter la validité, il faut accroître le réalisme, donc complexifier le modèle. La complexité rend toutefois plus difficile la création de la simulation, son utilisation et, ultimement, l'apprentissage qui en résulte (Fripp, 1993).

Par ailleurs, un certain niveau de réalisme est nécessaire pour garantir la valeur des apprentissages (Morris, 1986). Comme on peut l'observer dans la figure 12, le créateur de simulation doit donc trouver un moyen terme entre l'exhaustivité et la simplicité dans le choix des paramètres de la simulation qui en garantiront l'efficacité. 


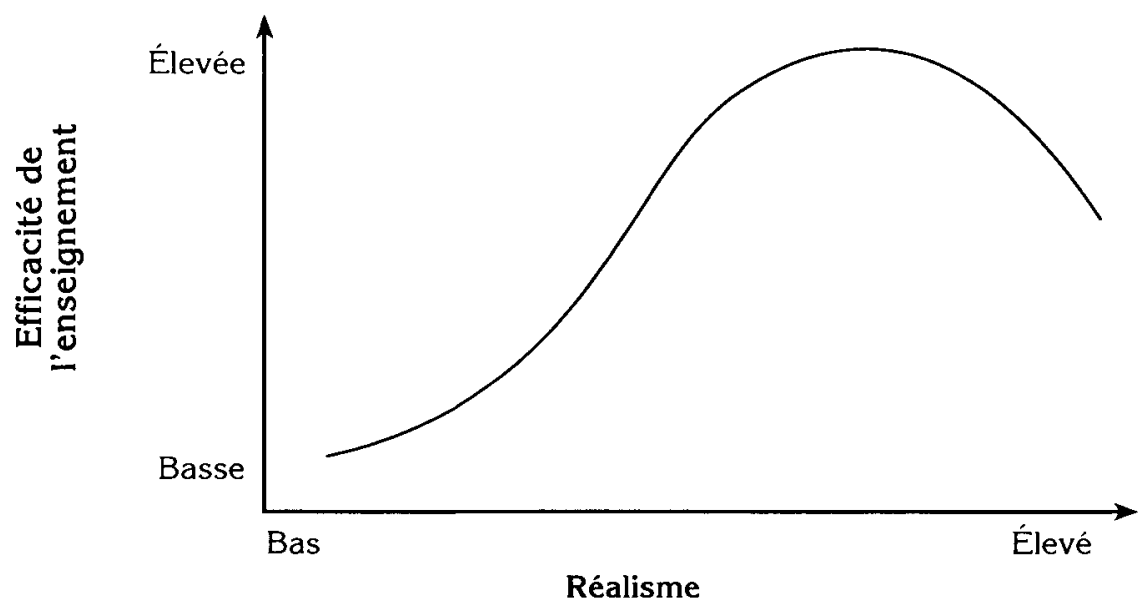

Figure 12: Relation entre le réalisme de la simulation et l'efficacité de l'enseignement (Norris, 1986, p. 449)

Finalement, il y a lieu de soulever un problème que pose la conception de simulation : une mauvaise simulation risque de fausser la réalité qu'elle décrit et mener à des apprentissages erronés. Et comme disait Einstein, « ce qui est grave, ce n'est pas ce qu'on ne sait pas, mais ce qu'on sait et qui est faux $»$.

\section{Avantages}

Outre les apprentissages spécifiques qu'elle permet de viser, la simulation peut contribuer à l'atteinte d'objectifs beaucoup plus larges étant donné que l'apprenant agit non seulement comme observateur de la situation mais aussi comme acteur. La simulation engage l'apprenant dans son apprentissage.

La simulation permet de s'affranchir de plusieurs contraintes du monde réel. Les phénomènes soit trop lents, trop rapides, trop rares ou trop dangereux deviennent accessibles. La simulation est pratique pour contourner les difficultés inhérentes à l'étude de certains sujets. 
L'apprenant peut apprendre beaucoup par essais et erreurs sans avoir à subir les conséquences de ses erreurs. La simulation confère une grande liberté à l'apprenant et stimule son jugement.

Par la rétroaction immédiate que le modèle fournit, la simulation est une des seules façons d'illustrer de manière dynamique les relations de cause à effet.

Comme l'illustre l'épigraphe attribué à Confucius en début de chapitre, l'apprentissage réalisé au cours d'une simulation est susceptible d'être mieux intégré et l'apprenant retient généralement mieux et plus longtemps l'objet d'étude.

En outre, la simulation est souvent caractérisée par des activités comme l'organisation, la concertation et la négociation. Les habiletés reliées à la communication y sont mises à contribution.

Bon nombre de simulations sont construites essentiellement autour de processus de solution de problème, d'où le potentiel de cette formule pour l'acquisition d'heuristiques ou de stratégies cognitives.

Comme il a l'occasion d'appliquer concrètement ce qu'il a appris théoriquement, l'apprenant peut évaluer jusqu'à quel point les apprentissages ont été intégrés. Par le fait même, la simulation lui permet de renforcer ces apprentissages. Cela comble un vide de bien des enseignements qui demeurent incomplets à cet égard. La séquence "intégration - renforcement - évaluation " ainsi obtenue est particulièrement fructueuse. 
Jeu, simulation et jeu de rôle

\section{Limites}

Le peu de disponibilité de simulations déjà construites représente un frein majeur à l'utilisation de cette formule. Les simulations en français sont rares.

La création d'une simulation force un compromis entre la simplicité et l'exhaustivité du modèle. Si le juste équilibre n'est pas obtenu, la simulation peut entraîner deux conséquences indésirables :

- trop simple, elle risque de fausser la réalité ;

- trop complexe, elle risque de créer une certaine confusion chez l'apprenant.

C'est une formule pédagogique qui requiert un certain temps de préparation, et l'activité elle-même est souvent assez longue à réaliser. Ce qui s'explique en quelques minutes dans un exposé magistral nécessitera parfois une heure ou plus dans l'esprit d'un apprentissage expérientiel comme celui réalisé à l'aide d'une simulation. L'enseignant ou le formateur doit évaluer au départ si l'apport supplémentaire à la qualité des apprentissages justifie le surplus de temps à y investir. Quoi qu'il en soit, la simulation nécessite un investissement de temps non négligeable.

Par ailleurs, concevoir une simulation et produire le matériel nécessaire prend, bien entendu, un temps considérable et entraîne souvent certains frais. Élaborer sa propre simulation est une entreprise assez lourde pour l'enseignant.

La qualité des apprentissages réalisés dépend souvent de l'exploitation qui en est faite par l'enseignant au moyen d'une discussion après la simulation. D'autres informations supplémentaires sont souvent nécessaires sinon il y a risque que 


\section{l'apprenant reste fixé sur des détails qui l'ont impressionné et ne parvienne pas à généraliser les principes sous-jacents.}

\section{Conditions d'utilisation}

Le choix d'une simulation doit être fait suivant les objectifs qu'on se propose d'atteindre. La première question à se poser est la suivante : " L'objectif visé se prête-t-il à l'enseignement au moyen d'une simulation ? » Car il faut bien l'admettre, tout ne peut être simulé.

En second lieu, il faut établir, comme nous le mentionnions plus haut, s'il vaut la peine d'investir le temps que cela requiert pour atteindre l'objectif en question; d'autres formules pédagogiques qui prennent moins de temps sont peut-être préférables. La même logique peut prévaloir quant aux dépenses éventuelles reliées à ce projet.

Enfin, il faut habituellement adapter la simulation pour qu'elle cadre avec le contexte dans lequel on choisit de l'employer. Dans bien des cas, il s'agit de modifier les activités de suivi proposées (et au premier chef, la discussion qui suit immédiatement la simulation) alors que la simulation comme telle reste relativement inchangée.

De fait, le principal problème réside dans le repérage des simulations existantes. Quelques périodiques spécialisés comme Simulation \& Games ont des chroniques où sont passées en revue les plus récentes simulations produites. Quelques firmes de production de matériel didactique disposent également de simulations dans leurs répertoires de produits alors que d'autres, comme la compagnie Simile II, se sont spécialisées dans ce type de matériel. 
Jeu, simulation et jeu de rôle

C'est surtout auprès des firmes de production de matériel informatique qu'on peut faire les meilleures trouvailles. En effet, une grande quantité de logiciels de simulation ont vu le jour à la faveur de l'avènement de la micro-informatique de masse.

En conclusion, la simulation est un bon outil pour l'apprentissage par la découverte. Elle est basée sur un modèle d'apprentissage inductif où l'étudiant fait face à une situation concrète qui lui permet de vivre une certaine expérience dont il tirera lui-même ses enseignements. Complétée par une discussion structurée correctement, la simulation, bien qu'onéreuse en temps, favorise des apprentissages plus significatifs pour l'apprenant que les autres types d'intervention pédagogique en général.

Modèle d'une réalité, la simulation doit y être fidèle jusqu'à un certain point et éviter de la fausser. Sa valeur, en tant que soutien pédagogique, varie suivant la qualité du matériel proposé et les compétences d'animateur dont saura faire preuve l'intervenant tant lors de la simulation qu'au cours de la discussion subséquente. Le chapitre 7 traite largement de cet important aspect lié au succès de la simulation dans un contexte d'enseignement ou de formation. 


\section{Exemples de simulations}

\section{« Répartition »}

par Guy Provost (adapté de Conner)

Type : Simulation humaine

Clientèle : Secondaire et collégial

Nombre de joueurs : 15 à 60 et plus

Durée : 20 à 30 minutes

L'objectif de «Répartition » est de permettre aux apprenants de visualiser la répartition des populations et des richesses sur la planète. Chaque participant pige dans un chapeau un carton de couleur lui indiquant à quel continent il appartient; la signification des couleurs n'étant pas révélée au départ, les participants sont invités à la découvrir. On retrouve ainsi $59 \%$ des participants en Asie, $17 \%$ en Europe, $10 \%$ en Afrique, $8 \%$ en Amérique latine et, enfin, $6 \%$ en Amérique du Nord ${ }^{1}$; il s'agit de la répartition réelle de la population dans le monde. On demande à chaque groupe-continent d'occuper un espace que l'animateur a pris soin de délimiter sur le plancher (un revêtement de carreaux facilite cette opération). On remarque rapidement que les individus qui sont en Amérique ont beaucoup plus d'espace que les Asiatiques ou les Européens. La figure 13 illustre le découpage du territoire et la répartition des populations sur chacun des continents.

1. Notons que $6 \%$ de 25 personnes donne 1,5 personne. Comme il ne peut évidemment y avoir de demi-personne, on arrondit à l'entier le plus près, soit deux. 
Jeu, simulation et jeu de rôle
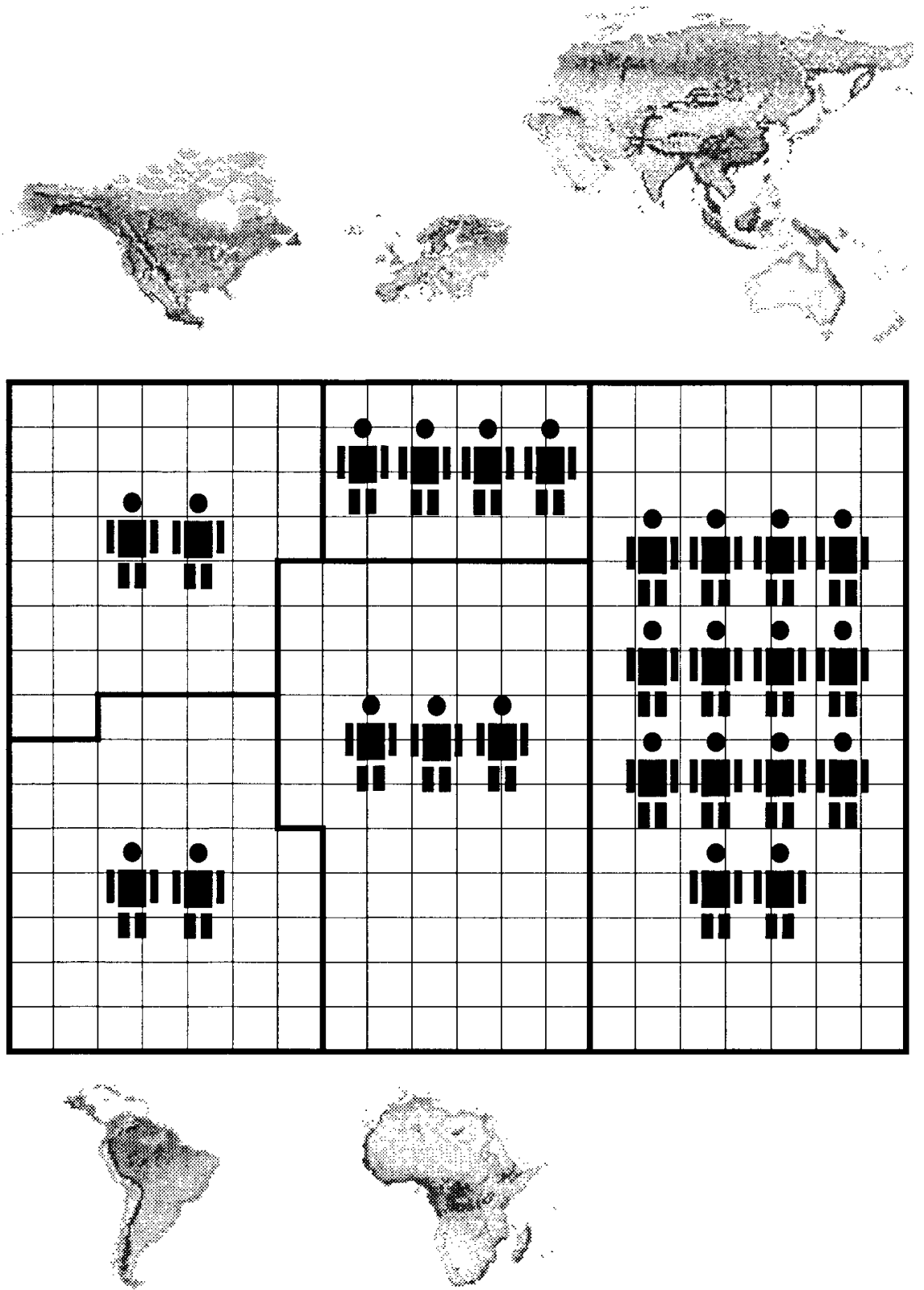

Figure 13: Territoires et populations tels qu'ils sont représentés dans "Répartition" avec 25 participants 
On distribue ensuite des chaises pour symboliser la richesse matérielle. En Afrique et en Asie, on se retrouve avec beaucoup moins de chaises que de personnes, alors qu'en Amérique du Nord on dispose de plus de trois chaises par personne (ces proportions sont tirées de la moyenne des produits nationaux bruts des pays du continent). Fait important à souligner, globalement il y a autant de chaises que de participants. On distribue ensuite de la nourriture toujours selon les proportions observables dans le monde : les Asiatiques sont nettement désavantagés par rapport aux NordAméricains et aux Européens. On retrouve à la figure 14 les quantités de chaises et de pommes à distribuer aux participants dans chaque continent pour représenter ces deux aspects. Notez qu'une chaise couchée représente une fraction de chaise et qu'une pomme croquée symbolise une fraction de pomme.

« Répartition » peut être adapté pour comparer deux, trois ou quatre pays seulement (par exemple, le Canada, l'Éthiopie, l'Inde et le Japon), mais l'utilisation des continents comme structure fournit une image plus saisissante puisqu'on fait abstraction de tout biais dans le choix des pays. En général, les participants ressortent fortement impressionnés par ce qu'ils ont vu L'effet dépasse largement ce qu'une démonstration théorique, même appuyée des schémas fournis ici, aurait permis. 
Jeu, simulation et jeu de rôle

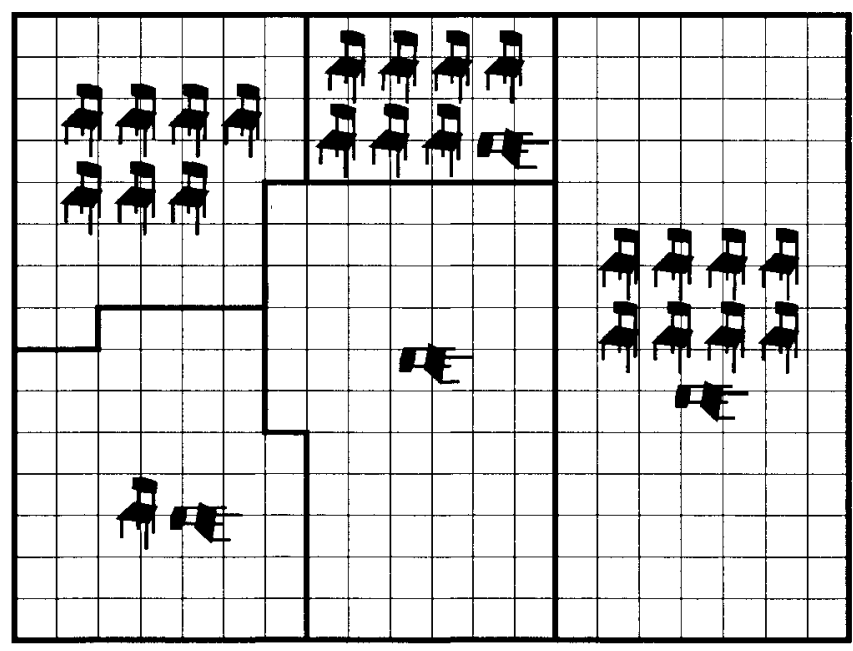

"RICHESSE

(moyenne

des PNB)"

"NOURRITURE (consommation de protéines)"

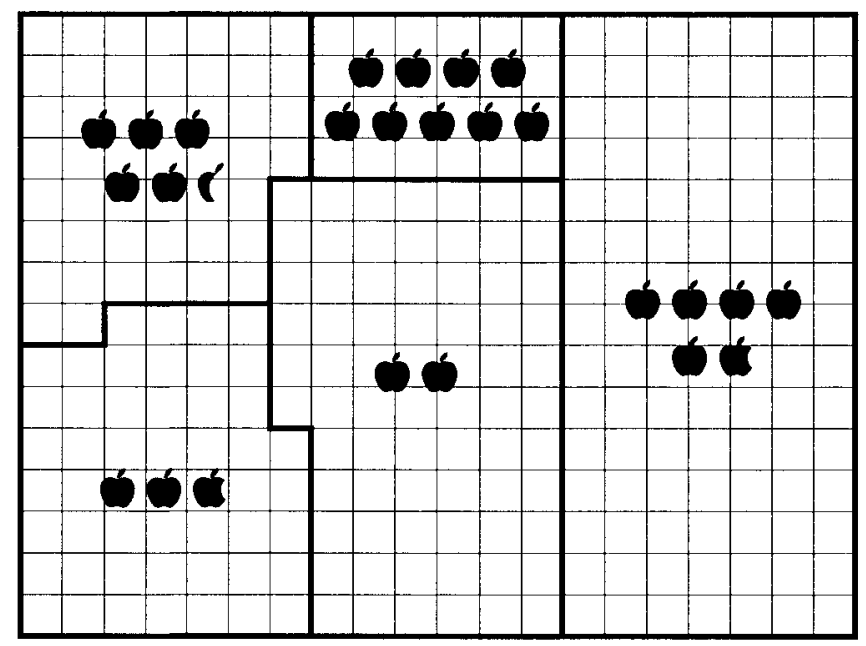

Figure 14: Répartition de la richesse et de la nourriture tels qu'ils sont représentés dans "Répartition" avec 25 participants 


\section{« SimCity 2000 »}

(logiciel par Maxis)

Type : Simulation informatique

Clientèle : Secondaire et collégial

(adaptable pour le deuxième cycle du primaire)

Nombre de joueurs : 1 à 3 par ordinateur

Durée: 20 à 30 minutes

« SimCity 2000 » est une simulation du développement urbain et de la gestion de ses différents paramètres. L'usager contrôle l'aménagement de zones résidentielles, commerciales ou industrielles, l'implantation de services de police, de protection des incendies, la construction de routes et la mise en place de l'électricité, etc. Parc nautique, stade de sport, etc., deviennent des équipements disponibles lorsque les revenus de taxe le permettent. L'ordinateur gère les dépenses occasionnées par les aménagements et fournit une rétroaction à la qualité des décisions. Ainsi, une zone résidentielle construite près des industries amènera un faible revenu de taxes, alors qu'en bordure d'un cours d'eau ce revenu sera nettement meilleur. La criminalité, les bouchons de circulation, etc., qui sont le lot des grandes villes sont également simulés et le gestionnaire doit appliquer les solutions appropriées.

Certains scénarios préprogrammés sont disponibles, chacun comportant son propre défi (celui de Berlin, une ville ravagée par la guerre, de San Francisco, aux prises avec un terrible tremblement de terre, ou d'une autre ville inconnue, caractérisée par la platitude !), et on peut provoquer des désastres à son gré (raz de marée, tornade, monstre marin !, etc.). On voit que certains éléments plus ludiques se greffent à la simulation, mais le modèle en demeure le point central. 


\section{«They Shoot Marbles, Don't They? »}

par Frederick L. Goodman et Robert Parnes

Type : Jeu de simulation intégrant la simulation et le jeu de rôle

Clientèle : Deuxième cycle du secondaire et collégial

Nombre de joueurs : 8 à 50

Durée : 2 heures et plus

Ce grand classique de la simulation a d'abord été développé par Fred Goodman dans le cadre d'une campagne auprès des policiers de Détroit pour juguler la corruption dans leurs rangs. "They Shoot Marbles, Don't They ? » est rapidement devenu une simulation " tous azimuts que chaque utilisateur teintait de sa touche personnelle, d'ailleurs au grand plaisir de son créateur. »

Lorsque l'activité commence, seules cinq personnes jouent aux billes et doivent réussir certains coups (c'est le travail) sur une surface de jeu d'environ un mètre carré. Une personne voit à l'application de certaines règles (c'est la police), une autre a le pouvoir d'ajouter, d'éliminer ou de modifier les règles (c'est le gouvernement) et un juge est nommé en cas de contestation d'une décision. Le tout se complique progressivement au fur et à mesure qu'apparaissent de nouveaux rôles : parti d'opposition, journalistes, entrepreneurs (qui possèdent les usines où les joueurs de billes " travaillent »), propriétaires fonciers (qui louent ou vendent des terrains pour la construction d'usines par les entrepreneurs), banquiers, assureurs, fraudeurs, etc. Ici, l'imagination de l'animateur est la seule balise qui tienne !

Une des grandes qualités de cette simulation tient au fait qu'elle commence très simplement et qu'elle se complique progressivement. On intègre les participants à l'activité à mesure qu'ils arrivent dans le local et ils doivent comprendre ce qui se passe en observant et en questionnant les autres. Tous se demandent où tout cela va les mener, ce qui est d'une part plutôt imprévisible et, d'autre part, assez peu important. Ce sont davantage le processus et les réactions des individus les uns vis-à-vis des autres qui ont une certaine valeur pédagogique. La discussion qui suit l'activité est, bien sûr, de toute première importance. 


\section{Chapitre 5 \\ Jeu de rôle}

Le monde entier est une scène dont nous sommes tous les acteurs.

William Shakespeare

\section{Définition}

Interprétation du rôle d'un personnage en situation hypothétique en vue de mieux comprendre les motivations qui justifient les comportements (Chamberland, Lavoie et Marquis, 1995, p. 71).

Le jeu de rôle repose sur une interprétation mais se distingue du jeu théâtral par l'absence de répliques toutes faites, de scénario précis et de décor. Si parfois la prestation des participants prend un air théâtral, celui-ci n'est qu'accessoire et ne constitue pas le but de l'exercice.

La situation est hypothétique et laisse beaucoup de latitude quant au dénouement de la scène ; un jeu de rôle est une activité très peu contraignante. Ses règles sont plus souples que celles du jeu et il n'est question ni de gagnant ni de fin prédéterminée.

L'objectif visé est une meilleure compréhension des motivations de l'être humain (ou, par extension, de l'animal). L'objet d'étude n'est pas la réalité mais bien les comportements qu'elle suscite. Soulignons que le jeu de rôle se distingue de la simulation par le fait qu'on ne cherche pas à modéliser la réalité ; 
Jeu, simulation et jeu de rôle

on s'y réfère, mais sans chercher à la reproduire avec justesse.

\section{Situation typologique du jeu de rôle}

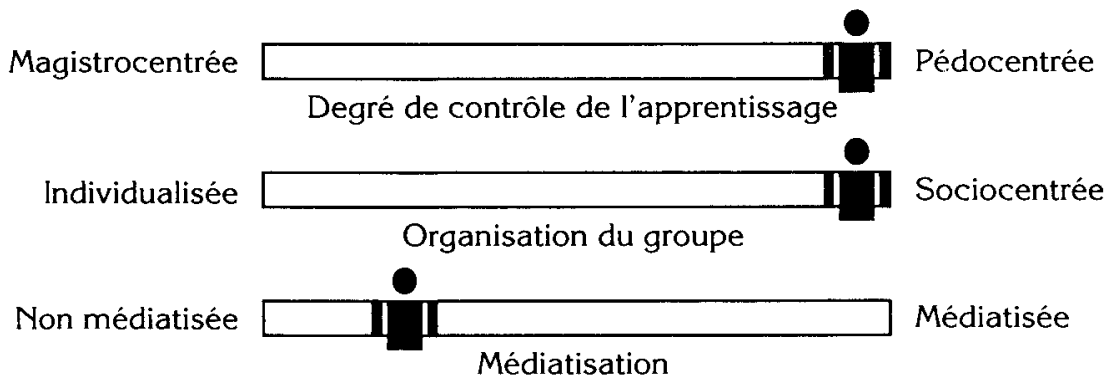

Le jeu de rôle est fortement pédocentré, car l'apprenant est, c'est le cas de le dire, l'acteur principal. Comme son nom l'indique, le jeu de rôle est construit autour de rôles assignés à des personnes. La formule est on ne peut plus sociocentrée, les autres apprenants agissant soit à titre de protagonistes, soit à titre d'observateurs. La formule est peu médiatisée, car la scène est souvent amorcée par une mise en situation simple et ne comporte habituellement pas d'accessoire précis.

\section{Origines du jeu de rôle}

Le fait de jouer à être quelqu'un d'autre correspond à une propension innée de l'être humain qui se manifeste dès le plus jeune âge. L'enfant s'y adonne spontanément, et cette forme d'activité devient pour lui un instrument de développement très important. 
Malgré le fait que l'on puisse identifier la dimension " rôle » dans bien des jeux, le jeu de rôle est en soi une forme de jeu spécifique où l'accent est mis sur le rôle à jouer contrairement à la position accessoire qu'il occupe dans les jeux de société, par exemple.

De toutes les techniques de jeux, c'est celle qui pose le moins de contraintes. Elle se caractérise par une structure globale, large, informelle. Il s'agit habituellement de se mettre dans la peau de quelqu'un d'autre et de se comporter comme cette personne le ferait dans une situation hypothétique. La popularité des soirées « Meurtres et mystères $^{1}$ " ou des séances de "Donjons et dragons ${ }^{2}$ " est révélatrice de l'attrait qu'exerce cette forme de jeu.

Sous l'appellation " jeu de rôle ", on peut retrouver un très grand éventail d'activités. Pour bien cerner sa place et son apport dans la formation, il importe d'abord de bien le situer dans le contexte psychothérapeutique dont il est issu en grande partie.

La découverte de la valeur thérapeutique du jeu de rôle est habituellement attribuée au sociologue et psychiatre viennois Jacob Levy Moreno (1889-1974). Au début des années 1920, Moreno participe à l'expérimentation de nouvelles voies pour le théâtre. Il débute en créant un " théâtre spontané " où l'on ne retrouve ni acteur, ni texte. Les spectateurs sont invités à monter sur scène et à jouer spontanément sur

1. Il s'agit de mises en scène dans lesquelles les participants tiennent un rôle et doivent tenter de dénouer une intrigue.

2. Rencontres (qui durent souvent plusieurs heures) préparées par un maître de jeu qui construit des scénarios pour des personnages aux pouvoirs magiques associés à chacun des participants qui décident comment réagir à des menaces ou à des événements prévus à l'avance. 
Jeu, simulation et jeu de rôle

un thème suggéré. Ce projet, très proche des ligues d'improvisation d'aujourd'hui, se révèle à l'époque un échec retentissant qui amène Moreno à revoir ce concept.

Il décide de passer d'une thématique générale à des faits particuliers. C'est ainsi que sera créé le journal vivant où des acteurs amateurs et des volontaires parmi les spectateurs improvisent à partir des faits divers ou sensationnels rapportés dans les journaux du jour. Il fait reconstituer sur scène, de manière spontanée, l'événement choisi. Au cours d'une de ces séances, en 1928, Moreno découvre l'effet cathartique du jeu de l'acteur. Voici, tel que cité dans Schützenberger (1981, p. 4-6), l'essentiel du « cas Barbara » relaté dans le journal de Moreno.

\section{Le cas Barbara}

J'avais créé près de l'opéra de Vienne un «théâtre de spontanéité » (Das Stegreiftheater), qui s'est transformé une nuit en un " théâtre pour la spontanéité » ou théâtre thérapeutique. Nous avions alors une jeune actrice, Barbara, qui travaillait pour le théâtre et qui prenait aussi part à une nouvelle expérience que j'avais entreprise, le journal vivant ». Elle avait beaucoup de succès, à cause de son talent: elle excellait dans les rôles romantiques, héroïques, d'ingénue. Il devint vite évident qu'elle était tombée amoureuse d'un jeune poète et auteur dramatique (Georges) qui ne manquait pas de venir toujours au premier rang l'applaudir. Un jour, on annonça leur mariage. Toutefois, rien ne changea, elle resta notre principale interprète, et lui notre plus fidèle spectateur, - si l'on peut dire.

Un jour, Georges vint vers moi, ses yeux, si gais d'habitude, préoccupés et tristes : «Qu'est-il arrivé ? » je lui demande. Oh, Docteur, je ne peux plus la supporter !» - «Supporter quoi ? »Je le regarde de façon pénétrante, cherchant, investigant. Cet être si doux, si angélique, que vous admirez tous, agit comme le diable en personne lorsqu'elle est seule avec moi. Elle jure, utilise des mots orduriers, et lorsque je me fâche, comme ça m'est 
arrivé hier, elle me tape avec ses poings. » — « Attendez, je lui dis, venez ce soir au théâtre avec elle comme d'habitude. Je vais essayer un truc. " Lorsque Barbara entre dans les coulisses, ce soir-là, prête à jouer un de ses rôles habituels de sainte et pure femme, je l'arrête au passage. « Écoutez, Barbara, venez voir. Vous avez été merveilleuse jusqu'ici, juste parfaite, mais j'ai un peu peur que vous vous répétiez et que ça lasse les gens à la longue. Les gens voudraient vous voir dans des rôles plus à ras de terre, plus proches de la nature humaine toute crue, toute vulgaire. Les gens de la rue sont malheureux, stupides, vulgaires, cyniques. Ce serait intéressant de montrer les gens, non seulement comme ils sont, mais pires qu'ils sont, les gens comme ils se montrent lorsqu'ils sont poussés à bout par des circonstances inhabituelles. Vous avez envie d'essayer ? » « Oui, répond-elle avec enthousiasme, je suis contente que vous l'ayez mentionné. Ça fait un petit moment que je ressens que je dois donner à notre auditoire du nouveau. Mais vous pensez vraiment que je peux y arriver ? \ - « Je vous fais entièrement confiance, je lui dis, on vient tout juste d'apprendre qu'une fille qui guettait le client à Ottakring (un bas quartier de Vienne), dans la rue, a été attaquée et tuée par un étranger. Il court encore ; la police le recherche. Tu es une « respectueuse » tu fais le trottoir... Ici (montrant du doigt Richard, un de nos acteurs), c'est l'apache. On y va. Préparez la scène. \ On improvise une rue sur scène, un café, deux lumières. À partir de là, Barbara improvise, Georges étant comme d'habitude au premier rang, tout excité. Richard, dans le rôle de l'apache, sort du café avec Barbara et la suit... Il y a une rencontre entre eux, qui se transforme très vite en discussion violente au sujet de l'argent. Soudain, Barbara se transforme totalement et joue d'une façon tout à fait inattendue d'elle. Elle jure comme un charretier, tape le gars, lui donnant des coups de pied dans les jambes. Je vois alors Georges se redressant à demi me montrant la scène avec anxiété, en levant un peu son bras. L'apache devient violent, brutal, course Barbara ; soudain, il saisit son couteau (un accessoire de théâtre) de la poche intérieure de sa veste. Il lui court derrière, en cercle, plus près, encore plus près. Elle joue si bien qu'on ressent qu'elle est terrorisée. Le public se lève et crie : "Arrêtez, arrêtez-les », mais l'apache ne s'arrête pas, Barbara est donc « assassinée », là. Après la scène, après tout cela, Barbara 
Jeu, simulation et jeu de rôle

exulte de joie ; elle embrasse Georges, et ils rentrent à la maison bras dessus bras dessous, comme en extase.

Georges vint me voir le lendemain. Il comprend immédiatement que c'est une thérapie. À partir de ce jour, je (Moreno) proposai à Barbara de jouer des rôles de ce genre, bas et profonds, des rôles de domestiques, vieilles filles solitaires, épouses délaissées cherchant leur revanche, odieuses fiancées, serveuses, tenancières... Georges vient me voir chaque jour pour m'en rendre compte. Après quelques séances, il me dit : " Il lui arrive quelque chose ! Et ce n'est pas rien ! »... « C'est une métamorphose, me ditil quelques jours plus tard, elle a encore quelques accès de colère à la maison, mais ils sont moins intenses. Ils sont aussi plus brefs et, quelquefois en plein milieu, elle se met tout à coup à sourire, parce qu'elle se souvient de scènes du même genre qu'elle a jouées sur scène, comme hier soir, et je ris moi aussi, avec elle, et en même temps, car moi aussi, je me rappelle le jeu. On dirait que nous nous voyons l'un l'autre comme dans un miroir psychologique. Quelquefois, elle commence même à rire avant de se mettre en colère, car elle sait exactement comment cela va se passer. Dans certaines circonstances, elle se cabre encore dans son attitude, elle donne encore de grands coups de gueule, mais beaucoup moins qu'avant. »

On aurait dit une catharsis provoquée par le rire, la bonne humeur et l'humour. Je continuai le traitement en confiant à Barbara des rôles soigneusement adaptés à sa situation de conflits personnels, à elle et à son mari, selon leurs besoins. Georges me parle régulièrement, il me dit l'effet que lui fait, à lui, ce qu'elle joue sur scène: il dit avoir acquis une meilleure compréhension de sa femme, d'une part au travers des scènes que je lui faisais jouer, et d'autre part en écoutant et intégrant les commentaires que j'en donne en fin de séance. "Voir ce qu'elle joue sur scène (sa 'production') me rend plus tolérant avec Barbara, moins impatient. \ Ce soir-là, quand il me dit ça, je dis alors à Barbara combien de progrès elle avait fait en tant qu'actrice et la félicite (Moreno félicite toujours ses acteurs ; Pavlov dirait qu'il leur donne un renforcement-conditionnement positif) et lui demande si elle n'aurait pas envie de jouer sur scène avec son mari Georges comme partenaire. 
Je leur demande alors à tous deux, un soir, s'ils accepteraient de jouer ensemble et de commencer une sorte de thérapie de couple, une thérapie interindividuelle. Ils acceptent et leurs duos, leurs dialogues improvisés, qui ressemblent de plus en plus à des scènes de leur vie privée, deviennent un élément permanent du programme. Ils jouent leurs deux familles, des scènes d'enfance, leurs projets, rêves et plans d'avenir. Après chaque séance, quelques spectateurs viennent vers moi, disant avoir été émus par les présentations-représentations de ce couple, plus profondément que par tout autre spectacle. C'était une catharsis du public Quelques mois plus tard, Barbara et Georges s'assoient seuls avec moi dans notre théâtre. Ils s'étaient trouvés l'un l'autre, ou plutôt chacun s'était trouvé, soi, et avait retrouvé, ou plutôt trouvé, rencontré, l'autre ! J'analysai l'évolution à la lumière des scènes qu'ils avaient jouées et leur expliquai pourquoi et en quoi ils avaient surmonté leurs conflits et leur racontai l'histoire de leur cure, séance après séance, de psychodrame.

Ces événements conduiront finalement Moreno à développer une nouvelle approche de la thérapie psychologique de groupe plutôt qu'individuelle. Ainsi, celui que l'on qualifie de père de la psychothérapie de groupe est à l'origine de deux formes très importantes de jeu de rôle : le psychodrame et le sociodrame.

Le psychodrame est un jeu de rôle où le participant joue ce qu'il est, ce qu'il pense, ce qu'il fait. Il est l'acteur de sa propre personne, de son vécu. Ce type de jeu de rôle sert soit à reconstituer un événement passé ou à imaginer ce qui n'a pas encore eu lieu.

La reconstitution d'une situation vécue antérieurement permet de reprendre contact avec les traumatismes ou autres chocs psychologiques qui n'ont pas été assumés. Le fait de revivre l'événement amène une meilleure compréhension et une certaine libération. À titre d'exemple, une personne qui vivrait avec le remords de ne pas avoir su verbaliser et manifester l'appréciation et l'affection qu'elle avait à l'égard de sa mère 
décédée pourra, en reconstituant à l'intérieur d'un jeu de rôle une rencontre avec celle-ci, s'en libérer.

La deuxième forme de jeu de rôle, le sociodrame, revêt les mêmes caractéristiques que le psychodrame quant aux aspects de l'expression des pensées et des émotions d'un individu par l'intermédiaire d'un jeu dramatique improvisé. Cette fois-ci, cependant, l'accent est mis sur des problèmes sociaux. Il sera beaucoup utilisé pour mieux faire saisir les problèmes de relations entre différents groupes sociaux : ouvriers et patrons, parents et enfants, gens de race blanche et de race noire.

Le jeu de rôle tel qu'on le retrouve maintenant dans l'éducation et la formation constitue une adaptation d'une pratique tant du psychodrame que du sociodrame. Sa perspective n'étant pas d'abord thérapeutique, les éducateurs et les formateurs éviteront les mises en situation où l'accent porte sur les problèmes personnels profonds, l'intériorisation intensive et l'emphase émotive. Seules les personnes dûment qualifiées et préparées à cette fin doivent s'adonner à ces activités qui conduisent à l'expression du plus intime des individus. Ces techniques, en apparence faciles d'utilisation, ne procurent de véritables bénéfices qu'entre les mains d'experts qui les connaissent bien et savent en exploiter les richesses. Le non-initié peut causer plus de tort que de bien en improvisant dans ce domaine.

\section{Caractéristiques}

En éducation et en formation, le jeu de rôle occupe une place importante. Facile à utiliser, habituellement, il permet une variation dans les stratégies d'intervention et surtout l'atteinte d'objectifs qui ne serait guère possible autrement. L'improvisation n'y revêt pas le même cachet que celle que l'on 
retrouve en psychothérapie. Étant donné les objectifs poursuivis, les improvisations seront souvent effectuées dans un cadre précis où le participant aura fait certaines recherches et pensé à ses stratégies. Il sera donc moins enclin à livrer uniquement son point de vue personnel.

À partir de deux grandes catégories, Van Ments (1983) distingue les jeux de rôle selon six fonctions :

- décrire ;

- démontrer ;

- pratiquer;

- réfléchir ;

- sensibiliser ;

- créer/s'exprimer.

Nous avons adapté cette typologie de Van Ments et considéré que le jeu de rôle pouvait être particulièrement utile pour atteindre quatre grands types d'objectifs correspondant à autant de catégories :

- l'acquisition d'habiletés techniques et de méthodes ;

- le développement d'attitudes ;

- la compréhension de faits et de principes ;

- l'expression et la créativité.

\section{Acquisition d'habiletés techniques et de méthodes}

Nous retrouvons, dans cette première catégorie, les jeux de rôle où l'objet est de démontrer comment quelqu'un se comporte dans une situation donnée afin de pouvoir l'analyser et, très souvent, l'imiter. Toute l'attention est centrée sur la technique présentée. 
On traite ici du développement d'habiletés comme celles requise dans la vente, dans la pratique d'une interview, dans l'exercice de gestes à poser et de propos à tenir pour porter secours à un blessé grave ou, encore, dans la pratique d'une langue seconde.

La théorie d'apprentissage social de Bandura (1969) est souvent considérée comme le fondement psychopédagogique de ce mode de formation. Pour Bandura, le «modeling », pierre angulaire de sa théorie, consiste à observer un modèle présentant les points critiques d'apprentissage et à les pratiquer par un jeu de rôle.

\section{Le jeu de rôle devient le banc d'essai où l'apprenant s'exerce en} vue de maîtriser l'habileté ou la technique visée. Cette fonction du jeu de rôle est probablement la plus utilisée. La pratique suit habituellement la démonstration dont on vient tout juste de parler. L'aspect improvisation, bien que toujours présent, n'y est pas prépondérant. Habituellement, l'apprenant s'exerce à suivre des étapes, une procédure, une manière d'agir. L'activité est plutôt structurée et méthodique. Cette utilisation correspond parfaitement à la phase de rodage (ou tuning) du modèle d'acquisition des compétences de Brien (1994).

En outre, les observateurs doivent être bien encadrés de manière à profiter pleinement de l'activité. Il est important de prévoir des feuilles d'observation pour analyser la performance de celui ou celle qui est en situation de pratique. Cela constitue pour l'observateur une excellente préparation à la pratique de l'habileté qui suivra.

L'enregistrement sur bande vidéo ou sonore peut aider grandement l'apprenant. Rien ne vaut de s'entendre ou de se voir en plus d'écouter les remarques et suggestions de ses compagnons et celles de l'enseignant. 


\section{Développement d'attitudes}

Dans une deuxième catégorie de jeux de rôle, la visée des apprentissages est d'un tout autre ordre. Il s'agit ici de se découvrir soi-même et de découvrir les autres. Les mises en situation sont habituellement organisées autour d'un problème et amènent les participants à se percevoir par rapport à celui-ci et à comprendre la position, les sentiments et les attitudes des autres. Pour mieux comprendre les attitudes de leurs parents envers eux, les adolescents peuvent se partager les rôles des parents et des adolescents et mettre en scène des événements susceptibles de faire valoir les points de vue respectifs.

La base de cette catégorie de jeux de rôle est la réflexion, prise d'ailleurs dans deux sens : celle de renvoyer une image de la réalité, comme dans le cas du miroir, et celle de penser, de chercher, de cogiter.

Dans le premier cas, l'objectif est de montrer à l'apprenant de quelle manière son comportement est perçu par les autres participants et l'animateur. C'est un aspect que l'on retrouve dans la majorité des jeux de rôle où il y a démonstration, comme nous l'avons vu dans la catégorie précédente. Par conséquent, une grande similitude existe avec les jeux de rôle axés sur la pratique, surtout ceux dont la demande prévoit ce type de feed-back par les pairs et l'usage de l'enregistrement vidéo. La distinction pourrait peut-être se faire à partir des intentions visées par l'activité. Ce qui importe ici, ce n'est pas tant de prouver que l'on maîtrise une technique, mais bien de saisir l'image qu'une personne projette.

Dans le second cas, la réflexion est prise dans le sens de la pensée, du questionnement. Afin de tenir son rôle dans le jeu avec un minimum de crédibilité, le participant doit d'abord réfléchir sur la situation particulière dans laquelle se trouve 
Jeu, simulation et jeu de rôle

le personnage qu'il incarne et se demander quel genre de réactions serait plausible compte tenu du scénario proposé. Ici, c'est le travail de préparation nécessaire à la performance du joueur qui constitue la réflexion souhaitée par le créateur du jeu.

Contrairement à la catégorie précédente, il ne s'agit pas ici de pratiquer une solution mais plutôt de rechercher des solutions. Sensibiliser les apprenants à un problème, à une situation, est souvent la première étape à franchir pour les amener à l'étudier et à le comprendre et, par voie de conséquence, à changer leurs propres perceptions de la réalité. Ici, nous touchons tant au potentiel de motivation que de compréhension accessible par le jeu de rôle.

Pour comprendre pleinement les problèmes des jeunes en milieu défavorisé, le simple fait de jouer à " Ghetto » ne sera pas suffisant. Cependant, cela permet d'amener le participant à mieux comprendre les déterminismes sociaux qui influent sur l'individu. Cette sensibilisation aux problèmes de gens vivant dans des milieux défavorisés se fait beaucoup plus facilement par la participation à un jeu de rôle évoquant l'ensemble de la réalité, avec les émotions et les difficultés inhérentes, que par n'importe quelle description. La fonction du jeu de rôle est de rendre des réalités perceptibles à l'apprenant tant sur le plan affectif qu'intellectuel. L'expression des émotions y est donc beaucoup plus importante que dans la catégorie précédente.

Les acquis sont, en outre, plus complexes et ne peuvent être évalués de la même manière que dans le cas de la maîtrise d'une technique. Notons, cependant, que ces deux catégories ne sont pas mutuellement exclusives : des éléments de l'une peuvent aisément se retrouver dans l'autre, car le jeu de rôle, comme la plupart des jeux, engage presque toujours toutes les composantes d'une personne. 
Très souvent, l'activité va occasionner chez le participant un engagement émotif et la manifestation d'attitudes qui seront l'objet principal du retour-synthèse ou debriefing sur lequel nous élaborerons au chapitre 8.

Le jeu de rôle axé sur les attitudes, particulièrement sur la réceptivité aux idées des autres, exige un certain doigté de la part de l'animateur. Son rôle dans la présentation, l'animation et l'exploitation du jeu est capital. Lorsqu'il est question d'émotions et de sentiments, une attention constante doit être portée quant à leur pertinence au jeu et à la poursuite des objectifs identifiés.

\section{Compréhension de faits et de principes}

La troisième catégorie regroupe les jeux de rôle visant la compréhension de faits et de principes. Leurs objectifs sont typiques des programmes scolaires: apprentissage de l'histoire, du fonctionnement du système politique, etc.

Les jeux de rôle de ce genre sont, par leur visée, à la frontière de la simulation. Il s'agit de ce que nous appelions dans l'introduction de cet ouvrage les jeux de simulations qui relèvent autant du jeu de rôle que de la simulation.

Ici, le jeu de rôle est utilisé comme moyen de communication pour décrire un problème, une situation. Compte tenu de leur caractère descriptif, les jeux de rôle de ce genre exigent une bonne préparation des participants. La reconstitution d'événements historiques ou d'une institution sociale (fonctionnement d'une banque, d'une compagnie, de l'Assemblée nationale, etc.) requiert une bonne dose de préparation. Car, il va sans dire, pour bien représenter ce qui est souhaité, il faut bien connaître la réalité que l'on veut décrire. L'apprentissage visé s'effectue tant par la recherche et la préparation à tenir 
Jeu, simulation et jeu de rôle

le rôle que par l'observation de la scène par ceux qui n'y participent pas directement. L'accent porte donc sur les faits, la réalité telle que la connaissent les participants, l'aspect de l'expression des émotions personnelles étant relégué au second plan.

Signalons que la pratique d'un jeu de rôle visant a priori la compréhension de faits et de principes entraînera certainement des acquisitions qui dépasseront ce contenu cognitif. Vivre la situation représente, pour l'apprenant, beaucoup plus qu'une simple description verbale ou écrite du sujet à l'étude. L'implication du participant, ou le rôle qu'il y joue, lui donne une perspective tout à fait particulière qui lui permet de percevoir des dimensions d'attitudes, de relations humaines, d'efficacité du système qu'il n'aurait pu saisir autrement.

Les jeux de rôle de cette dernière catégorie se caractérisent par la recherche ou la préparation qu'ils exigent. Bien que l'improvisation en fasse partie, celle-ci doit se dérouler à l'intérieur d'un cadre bien précis. Cela nécessite habituellement une recherche d'informations pour indiquer au participant ce qu'il doit en bonne partie représenter. Pour simuler le système judiciaire par un jeu de rôle, le participant doit véritablement apprendre son rôle, connaître ses caractéristiques, les limites de son pouvoir, les principes qui le gèrent. La préparation du jeu de rôle constitue, dans ce cas, une activité d'apprentissage menée souvent dans le cadre d'une recherche en bibliothèque ou d'une enquête qui se révèle très riche en retombées sur le plan des connaissances. Le jeu de rôle peut être perçu comme l'élément motivateur visant des apprentissages qui se font en grande partie avant même sa réalisation. 


\section{Expression et créativité}

Enfin, les jeux de rôle peuvent être utiles pour développer les capacités de création ou d'expression d'une personne. La facture typique du jeu de rôle, caractérisée par une structure très souple où le participant doit souvent prendre beaucoup d'initiatives, est propice à la génération d'idées et de stratégies tout comme à l'expression des émotions.

Plusieurs jeux de rôle ne sont que des mises en situation qui facilitent et souvent stimulent l'expression de l'individu en faisant appel à ses valeurs et à ses idées les plus personnelles. Les jeux visant à cerner les problèmes d'ordre éthique, par exemple « Bateau de sauvetage $\mathrm{s}^{3} »$, amènent non seulement le participant à s'exprimer sur des aspects fondamentaux de sa propre philosophie de la vie mais aussi à proposer des solutions nouvelles à des problèmes très difficiles à résoudre.

La création ne peut se faire à vide; elle émerge d'une bonne connaissance de l'art ou de la science dans laquelle elle s'exerce. Les jeux de rôle sont souvent vus comme d'excellents outils pour étudier les problèmes de relations humaines et de communication dans les organisations dans une perspective de recherche de solutions. Ils permettent de trouver des solutions à des problèmes complexes que plusieurs autres formes d'analyses traditionnelles ne permettent pas. C'est du moins la qualité qu'attribuent au jeu de rôle les professionnels œuvrant dans des multinationales de grande renommée (Duke et Mattley, 1986).

3. Il s'agit d'un exercice de prise de décision en groupe. Partant de l'hypothèse que seul un certain nombre d'individus peut survivre à une catastrophe, on doit choisir quelles personnes seront sacrifiées et pour quelles raisons. 


\section{Avantages}

Le jeu de rôle fournit un feed-back direct et immédiat à l'apprenant dans un contexte plaisant et différent des situations d'apprentissage habituelles. Le jeu de rôle est motivant.

Il permet d'aborder les attitudes dans un contexte sécurisant et contrôlé. Il constitue l'une des rares occasions pour l'apprenant d'extérioriser ses sentiments et de faire valoir ses idées aussi ouvertement en classe. Celui-ci apprend de plus à composer avec la frustration et à réagir aux situations de conflits. Le jeu de rôle procure un environnement propice à l'expression des idées et des émotions.

La majorité des apprentissages sont limités aux niveaux de la connaissance et de la compréhension. Il en résulte que les facultés reliées à l'application, à l'analyse, à la synthèse et à la critique sont peu sollicitées par les formules pédagogiques courantes. Le jeu de rôle offre une occasion d'application pratique et favorise l'atteinte d'objectifs de niveaux supérieurs.

Interpréter un autre point de vue que celui qui nous est naturel est un puissant facteur d'ouverture d'esprit. L'apprenant est forcé de s'interroger lorsqu'il décroche de sa perspective habituelle afin d'articuler ses idées et ses gestes en fonction de contraintes et de motivations différentes des siennes. En ce sens, le jeu de rôle sensibilise l'apprenant tout en le rendant empathique.

L'ambiance détendue qui s'installe dans la classe est très saine pour l'évolution du groupe et contribue à raffermir les liens entre chacun des membres. Le jeu de rôle contribue à établir des liens de complicité entre les apprenants et un bon climat dans la classe. 
Le jeu de rôle offre la possibilité à l'apprenant de se faire valoir et de développer sa créativité.

\section{Limites}

L'engouement exagéré de certains pour le jeu de rôle et l'exutoire que d'autres y voient engendrent un niveau sonore élevé et des écarts de langage parfois déplorables. La discipline peut être difficile à maintenir dans le groupe.

L'organisation d'un jeu de rôle est exigeante pour l'enseignant. La préparation, le déroulement et l'exploitation pédagogique qui suit l'activité prennent relativement plus de temps que les autres formes d'intervention pédagogique. Le jeu de rôle est exigeant en matière de temps et d'énergie.

Le jeu de rôle nécessite un bon encadrement des apprenants, autrement il y a risque de dérapage et de confusion. La qualité du jeu de rôle est tributaire des capacités d'animateur de l'enseignant.

Certains apprenants peuvent se retrancher derrière un refus de participer ou traiter la situation tellement à la légère qu'on passe à côté de l'objectif. Il y a risque que certains apprenants soient trop négatifs, trop gênés ou trop frivoles pour participer efficacement.

La profondeur avec laquelle le sujet sera traité est directement liée à la capacité des participants de puiser dans leur expérience et de la canaliser dans la mise en situation. Tous n'ont pas l'habileté intellectuelle de se concentrer sur l'essentiel ou le talent de l'exprimer efficacement. Le jeu de rôle peut banaliser la réalité et attirer l'attention sur des détails présentant peu d'intérêt. 


\section{Conditions d'utilisation}

Le jeu de rôle est une activité qui doit être soigneusement préparée. L'enseignant doit avoir une idée claire de ce qu'il veut accomplir avec le jeu de rôle. Il convient généralement d'informer les apprenants des objectifs poursuivis afin de les guider à moins qu'on ne souhaite justement en faire un élément de surprise. Il faut bien préparer l'activité et en communiquer le but aux apprenants.

L'enseignant doit être explicite quant à ses attentes. Ses consignes doivent guider les apprenants afin qu'ils évitent de tomber dans le piège de la prestation théâtrale ou des pitreries. Le jeu de rôle exige un bon encadrement de la part de l'enseignant.

Certaines balises doivent être respectées quant à la manière dont l'enseignant intervient. Ainsi, il doit :

- éviter de forcer qui que ce soit à participer ;

- démontrer sa confiance aux participants ;

- éviter d'être autoritaire ou de tenter de contrôler le déroulement ;

- limiter la durée à la juste mesure (souvent 5 à 10 minutes suffisent).

Un jeu de rôle ne devrait pas traîner en longueur. Il convient d'y mettre fin dès que l'effet recherché s'est produit. Certains excellents exercices à base de jeu de rôle durent à peine 5 ou 10 minutes. Les jeux de rôle de courte durée sont souvent les plus efficaces.

L'environnement physique doit être approprié au déroulement de l'activité, ce qui peut exiger un changement de local : on doit 
pouvoir s'exprimer sans crainte de déranger et sans être soi-même distrait. Il faut choisir un endroit qui permet à la fois de se concentrer et de s'exprimer.

Le jeu de rôle n'est qu'un élément d'une séquence d'enseignement efficace. Ainsi, il peut n'y avoir aucun bénéfice pédagogique réel si l'activité n'est pas suivie d'une discussion bien orientée. Il faut dégager les principes fondamentaux illustrés par le jeu de rôle et fournir de nouveaux exemples pour éviter que les apprenants ne conservent qu'un souvenir anecdotique de l'activité. Le jeu de rôle doit être complété par une discussion afin de faire ressortir les éléments clés de l'objet d'étude.

Comme nous l'avons mentionné auparavant, il est toujours important de situer la place du jeu de rôle dans le processus d'intervention dont il fait partie. Le jeu de rôle peut remplir différentes fonctions dans la planification d'une séance de formation ou d'un cours, tant sur le plan de la motivation, de l'acquisition ou de la performance. Il faut situer la contribution attendue du jeu de rôle dans la séquence d'enseignement.

\section{Organisation de la classe}

Indépendamment de leur appartenance à l'une ou l'autre des quatre catégories dont nous avons parlé plus haut, les jeux de rôle peuvent être organisés dans la classe de différentes façons. Quatre techniques peuvent être utilisées pour organiser le jeu de rôle :

- la technique centralisée ;

- l'aquarium ;

- le théâtre-forum ;

- la technique décentralisée. 
La figure 15 illustre les quatre types d'organisation de la classe tels qu'ils sont présentés dans 20 formules pédagogiques (Chamberland, Lavoie et Marquis, 1995, p. 73-75).

Dans la technique centralisée, chaque membre du groupe-classe tient un rôle, ce qui permet à tous de participer activement. Toutefois, cette forme perd de son intérêt à mesure que la taille du groupe augmente, car la confusion s'installe rapidement lorsque les protagonistes sont trop nombreux.

La technique de l'aquarium consiste à attribuer des rôles à quelques individus seulement alors que les autres agissent comme observateurs. Elle offre la possibilité d'une discussion enrichie des commentaires des protagonistes, très engagés et émotifs, et de ceux des observateurs, plus détachés et analytiques puisqu'ils jouissent d'un certain recul.

Pour les groupes où une grande confiance mutuelle existe déjà, le théâtre-forum constitue une option intéressante au lieu de l'aquarium. L'organisation y est similaire mais chacun des observateurs a le privilège d'arrêter l'action en cours pour se substituer à l'un ou l'autre des protagonistes du jeu de rôle. Le nouveau joueur modifie alors la dernière réplique pour imprimer un nouvel élan ou une nouvelle direction à la situation.

Enfin, la technique décentralisée permet de faire participer tous les membres du groupe-classe à la faveur de sous-groupes procédant simultanément à leur mise en situation. Contrairement à la technique centralisée, la cohésion est maintenue par un nombre restreint de protagonistes dans chaque groupe, mais la coordination de l'ensemble est compliquée d'autant. 


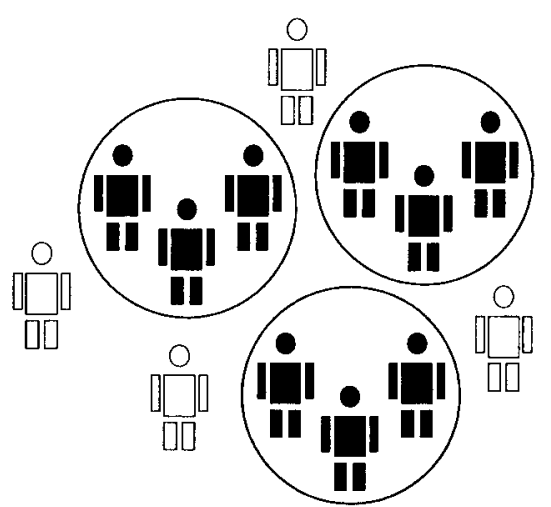

Technique "centralisée "

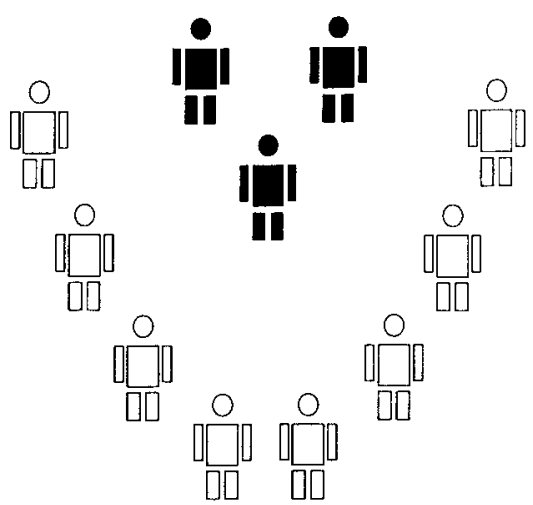

Technique de l'"aquarium "

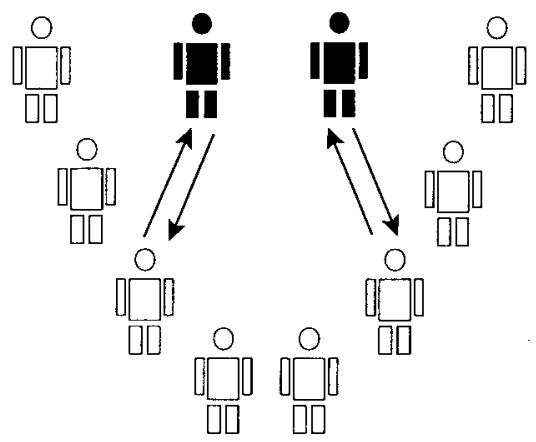

Technique du "théâtre-forum "

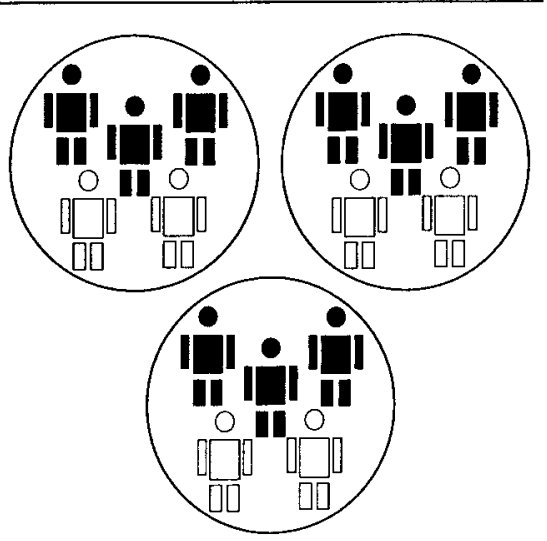

Technique " décentralisée "

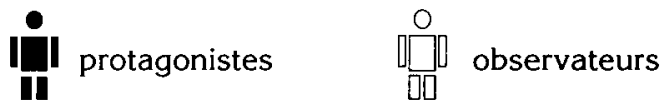

Figure 15: Organisation physique de la classe pour le jeu de rôle selon les techniques "centralisée", de l' aquarium", du "théâtre-forum " et "décentralisée" 
Jeu, simulation et jeu de rôle

\section{Exemples de jeux de rôle}

\section{« Rencontres du $13^{\mathrm{e}}$ type »}

par Gilles Chamberland (adapté de Greenblat)

Catégorie : Acquisition d'habiletés

Organisation : Technique décentralisée (3 personnes par équipe, jusqu'à 15 équipes)

Clientèle : Secondaire

Durée : Entre 5 et 10 minutes par situation, 3 à 6 situations une à la suite de l'autre

Le but de «Rencontres du $13^{\mathrm{e}}$ type » est d'apprendre à faire face à des situations corsées tout en trouvant des façons de contourner certains obstacles typiques à la communication. C'est une adaptation de «Close Encounters of the Difficult Kind », développé par Cathy Greenblat ${ }^{4}$ pour contribuer à réduire le taux élevé de grossesses indésirées chez les adolescentes de milieu défavorisé.

Les apprenants sont divisés en équipes de trois qui exécuteront toutes le même jeu de rôle simultanément. Dans chaque équipe, une personne est désignée pour être observateur pendant que les deux autres interprètent une courte scène après avoir consulté une carte décrivant la situation. Ces cartes décrivent, cependant, la situation à partir de points de vue fort différents de sorte que le conflit qui couve éclate. De plus, un des deux protagonistes pige une " carte de style » décrivant un trait de caractère ou un comportement irritant qu'il doit adopter. Cela constitue une barrière à la communication qui complique d'autant l'entente entre les deux personnages. L'observateur, quant à lui, ne reçoit aucune information écrite et tente de découvrir les données essentielles du problème. and Gaming Association tenue à Sherbrooke, Québec, en octobre 1987. 
Après chaque scène, il y a discussion en grand groupe au cours de laquelle l'animateur demande aux observateurs de partager leurs perceptions des faits illustrés. Deux personnes parmi les protagonistes lisent ensuite à haute voix la description de leur rôle, ce qui suscite un certain étonnement chez certains. Une brève discussion suit.

Un exemple des descriptions de rôle est fourni ici. De plus, trois exemples tirés des 13 cartes de style sont présentés à la figure 16 . 
Jeu, simulation et jeu de rôle

\section{"Rencontres} du $13^{e}$ type"

\section{Les catacombes}

de la mort

Rôle A

Tu es Pierre-Émile (4 $4^{\mathrm{e}}$ secondaire) et tu es responsable de l'équipe de production de la journée spéciale de l'Halloween de ton école. Cette équipe a la responsabilité d'approuver les projets soumis par les autres élèves et certains critères bien précis doivent être appliqués. Cette journée est vraiment une grosse affaire; l'an passé, TQS est venue filmer les meilleures activités.
Celle que tu rencontres est Laurence ( $3^{\mathrm{e}}$ secondaire), la meilleure amie d'Éloïse, ton ex-petite amie (c'est elle qui a mis fin à votre relation avant l'été). Laurence et toi, vous vous connaissez très bien mais vous ne vous êtes pas vus souvent depuis le début de l'année scolaire.

Laurence et cinq de ses amis (mais pas Éloïse) avaient soumis un excellent projet pour la journée spéciale : "Les catacombes de la mort. » Ils voulaient organiser un circuit dans le sous-sol de l'école où il y a les fournaises, etc., et faire passer les élèves dans des dédales de corridors faits avec des draps. Le tout se serait déroulé dans la noirceur presque totale (seulement quelques " blacklights ») et une musique d'horreur était prévue au programme.

Cependant, il y avait un problème de sécurité. Le circuit prévoyait un passage où on risquait de se cogner la tête sur des tuyaux et où il $\mathrm{y}$ avait aussi danger que les draps prennent feu, étant donné qu'à un certain endroit, l'espace est plutôt étroit entre deux fournaises. La moitié des membres du comité étaient d'avis qu'il fallait rejeter le projet pour ces raisons. Par contre, l'autre moitié considéraient que le projet méritait d'être approuvé, car, selon eux, on avait exagéré la question de la sécurité.

De plus, ils trouvaient, avec raison d'ailleurs, que c'était de loin le projet le mieux présenté de $3^{\mathrm{e}}$ secondaire. Il n'y avait pas vraiment de possibilité de modifier le projet. Tu as finalement donné ton avis et ton vote a fait pencher la balance, : le projet a été refusé. Ça tombait mal, car Laurence est la meilleure amie d'Eloïse. Depuis qu'elle a rompu avec toi, Eloïse t'en a fait arracher. Elle veut te faire passer pour un macho qui veut tout contrôler. Tu marches sur des oeufs même quand tu as affaire à ses amis, comme c'est le cas ici, car c'est Laurence qui a pris le leadership du projet des « catacombes de la mort ». La voici à la sortie de l'école; elle vient vers toi. 


\begin{tabular}{l|l}
$\begin{array}{l}\text { "Rencontres } \\
\text { du } 13^{e} \text { type" }\end{array}$ & $\begin{array}{l}\text { Les catacombes } \\
\text { de la mort }\end{array}$ \\
\hline Tu es Laurence ( $3^{\mathrm{e}}$ secondaire). Tu & $\begin{array}{l}\text { Celui que tu rencontres est Pierre- } \\
\text { Émile (4e secondaire). Tu le connais } \\
\text { bien, car c'est l'ex-petit ami } \\
\text { es très créative et tu as monté un } \\
\text { projet vraiment super pour la } \\
\text { journée spéciale de l'Halloween de } \\
\text { ton école: "Les catacombes de la } \\
\text { elle qui a cassé). Pierre-Émile est le } \\
\text { responsable de l'équipe de produc- } \\
\text { une grosse affaire à ton école; l'an } \\
\text { passé, TQS est venue filmer les la journée spéciale. C'est eux } \\
\text { meilleures activités. }\end{array}$ \\
\end{tabular}

Toi et cinq de tes amis (mais pas Éloïse) aviez soumis sûrement le meilleur projet de toute l'école pour la journée spéciale : "Les catacombes de la mort. » Il s'agissait d'un circuit dans le sous-sol de l'école où il y a les fournaises, etc. L'idée était de faire passer les élèves dans des dédales de corridors faits avec des draps. L'éclairage aux " blacklights " permettait de voir juste assez dans le noir et une musique d'horreur complétait le tableau.

Cependant, il y a eu un problème : Pierre-Émile a tout fait pour que le projet ne passe pas, question de sécurité, supposément. Il a influencé les plus faibles du comité pour qu'ils votent comme lui contre le projet. Les autres ont eu beau lui expliquer qu'il n'y avait pas de danger de se cogner la tête sur des tuyaux puisque deux élèves déguisés en sorcières s'occupaient de dire aux gens de se pencher aux endroits en question; rien à faire, il ne voulait rien savoir. Pierre-Emile aurait poussé le ridicule jusqu'à dire : "Y vont mettre le feu avec leurs couvertes. »

C'est un des membres du comité qui l'a raconté à Éloïse, qui n'est pas sur- prise du tout de cette attitude de son ex. " Tout ce qu'il peut faire pour se venger, il le fait », a-t-elle ajouté en te transmettant l'information. Pour ta part, tu trouves assez spécial qu'il s'en prenne à toi, qui n'es qu'une amie d'Elö̈se. Tu le vois qui sort de l'école. Il voudrait bien t'éviter, mais tu ne le laisses pas s'en sauver aujourd'hui. Tu vas à sa rencontre et tu lui adresses la parole en premier. 
Jeu, simulation et jeu de rôle

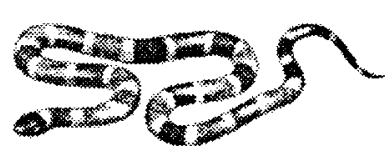

\section{Le serpent}

Le serpent se déplace en rampant grâce aux mouvements ondulatoires remarquablement coordonnés de son corps. Il se faufile ainsi à travers toutes sortes d'obstacles et atteint son but après plusieurs détours.

Durant la conversation, tu utilises des détours, tu "tournes autour du pot ". Tu ne laisses pas la chance à l'autre de comprendre exactement ce que tu veux dire, mais tu l'obliges plutôt à le deviner.
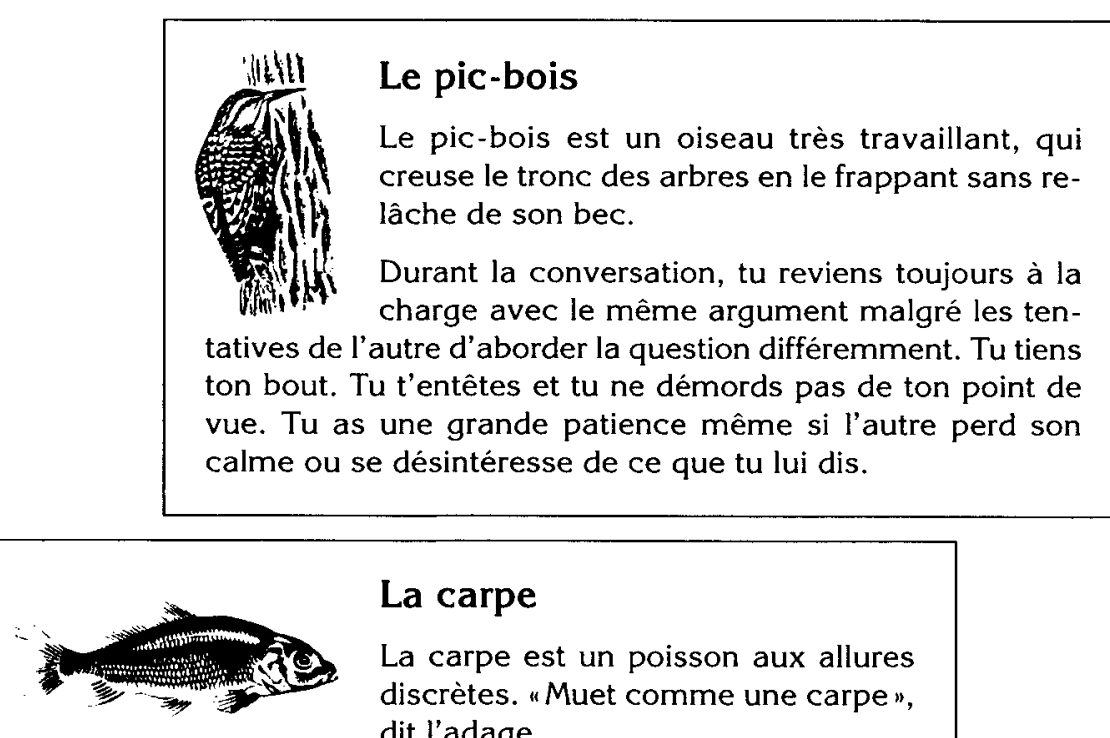

\section{La carpe}

La carpe est un poisson aux allures discrètes. "Muet comme une carpe", dit l'adage.

Durant la conversation, tu parles très peu. Tu réponds au minimum à l'autre et tu ne prends aucune initiative. Tu tolères très bien les silences, aussi longs soient-ils. Quand tu parles c'est avec un ton de conclusion, comme s'il n'y avait plus rien à dire d'un côté comme de l'autre. Tu persistes même si ça choque l'autre.

Figure 16: Exemples de cartes de style tirées de "Rencontres du $13^{e}$ type" 
Jeu de rôle

L'alternance rapide entre l'interprétation des scènes et les brèves discussions rend particulièrement agréable ce genre de jeu de rôle. On peut attirer l'attention sur un aspect à la fois et on élimine toute confusion quant au message véhiculé par le jeu de rôle. 
Jeu, simulation et jeu de rôle

\section{«Bonhomme-bonhomme »}

par Gilles Chamberland

Catégorie : Expression et créativité

Organisation : Théâtre-forum

Clientèle : Premier cycle du primaire

Durée : 10 à 12 situations limitées à 1 ou 2 minutes chacune, plus les interruptions

"Bonhomme-bonhomme » a pour but de permettre aux enfants d'exprimer certains sentiments et d'adopter des comportements simples. Ce jeu de rôle leur offre aussi l'occasion de créer de petites histoires à partir de quelques accessoires seulement.

Chaque enfant pige une carte à l'effigie d'un personnage de la série Bonhomme (voir figure 17) ; lorsque vient son tour, l'élève joue le rôle de ce personnage. L'enseignant choisit deux joueurs et leur donne quelques consignes verbales sur la situation à interpréter. Il agit également comme narrateur pour relancer l'histoire lorsque la situation s'enlise ou devient confuse. De plus, il intervient environ à toutes les deux minutes (ou plus fréquemment selon l'évolution de la scène en cours) pour procéder à une substitution de joueurs et en profite pour ajouter ou enlever un accessoire (brosse à dents, balai, pomme, chapeau, etc.). Le nombre de joueurs actifs simultanément est limité à trois. Un exemple de séquence de substitution de joueurs en fonction du temps est présenté au tableau 3.

La scène se poursuit ainsi jusqu'à ce que tous les élèves soient passés. On peut faire lire les livres par les élèves la veille ou la journée même pour fournir un plus grand répertoire de comportements à utiliser en cours de jeu. Une variante consiste à demander aux élèves de deviner le rôle interprété par chacun ; on aura alors eu soin de demander auparavant que 
chacun garde le secret sur le nom de son personnage.

Tableau 3: Exemple d'ordre de substitution des joueurs dans le jeu de rôle "Bonhomme-bonhomme "

\begin{tabular}{l|c|c|c|c|c|c|c|c|c}
\hline Minutes & $\mathbf{1}$ & $\mathbf{2}$ & $\mathbf{3}$ & $\mathbf{4}$ & $\mathbf{5}$ & $\mathbf{6}$ & $\mathbf{7}$ & $\mathbf{8}$ & $\mathbf{9}$ \\
\hline Monsieur & Petit & \multicolumn{2}{|c|}{ Curieux } & \multicolumn{2}{|c}{ Bavarde } & \multicolumn{2}{|c}{ Mal poli } & \multicolumn{3}{|c}{ Indécise }
\end{tabular}

ou

Madame ...

\section{Dodue}

Beauté

\section{Peureux $\quad$ Risette}

Pressé
Farfelu

Magie
Chipie

Lent

Le jeu de rôle est suivi par une discussion durant laquelle on explore les aspects positifs et les aspects négatifs de chaque trait dépeint par les personnages. L'enseignant guide la discussion pour éviter que les commentaires soient des jugements sur la prestation des élèves pour lesquels un rôle de composition peut parfois être assez difficile à tenir.

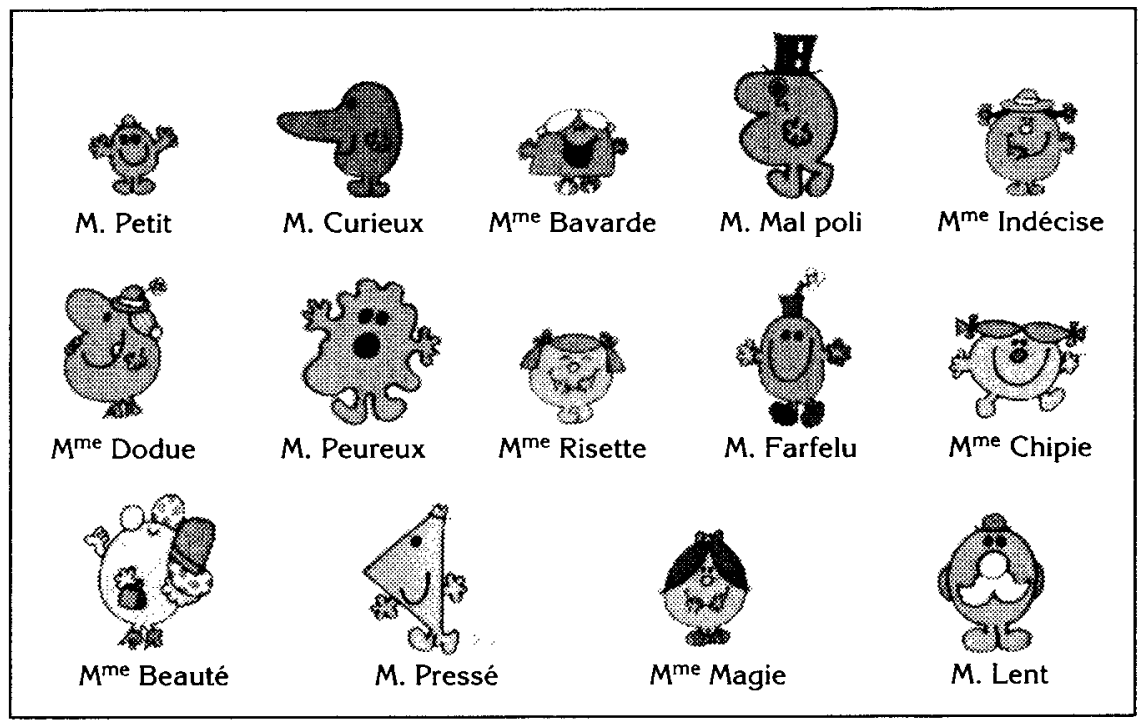

Figure 17: Quelques-uns des personnages de la série "Bonhomme" 
Jeu, simulation et jeu de rôle

\section{«Alerte à l'environnement »}

par Gilles Chamberland (adapté de Troyka et Nudelman)

Catégorie : Compréhension de faits et de principes

Organisation : Aquarium

Clientèle : Secondaire et collégial

Durée : 3 à 5 séances de 50 minutes chacune

Ce jeu de rôle est une adaptation de «Conservation Crisis », tiré du recueil Taking Action (Troyka et Nudelman, 1975). Les auteurs visaient surtout à fournir une occasion de parfaire les habiletés d'écriture, de lecture, d'expression orale et d'écoute. Mais « Alerte à l'environnement » cherche plutôt à faire ressortir la difficulté de concilier les intérêts divergents de certains groupes d'un milieu donné. On tente ainsi d'élargir la vision des joueurs et de les obliger à composer avec des contraintes imposées par les défenseurs d'autres intérêts que les leurs.

L'histoire se déroule à Sainte-Fictive, une petite municipalité au bord de la mer dont les deux moteurs économiques sont le tourisme et une grande usine de transformation. Or, l'usine pollue la baie ; par conséquent, le sanctuaire d'oiseaux marins est menacé et l'industrie touristique subit les contrecoups d'une mauvaise réputation en matière d'environnement. Par ailleurs, la fermeture de l'usine aurait de graves répercussions se traduisant par des pertes d'emploi. La solution de l'enfouissement des déchets toxiques dans un site propice est contestée à la fois par les Autochtones d'une réserve avoisinante et par le lobby d'un groupe de résidents d'une banlieue cossue, le secteur Bellevue. C'est le vieux réflexe du pas dans ma cour» qui se manifeste ici. La figure 18 représente la situation géographique de la municipalité. 
Jeu de rôle

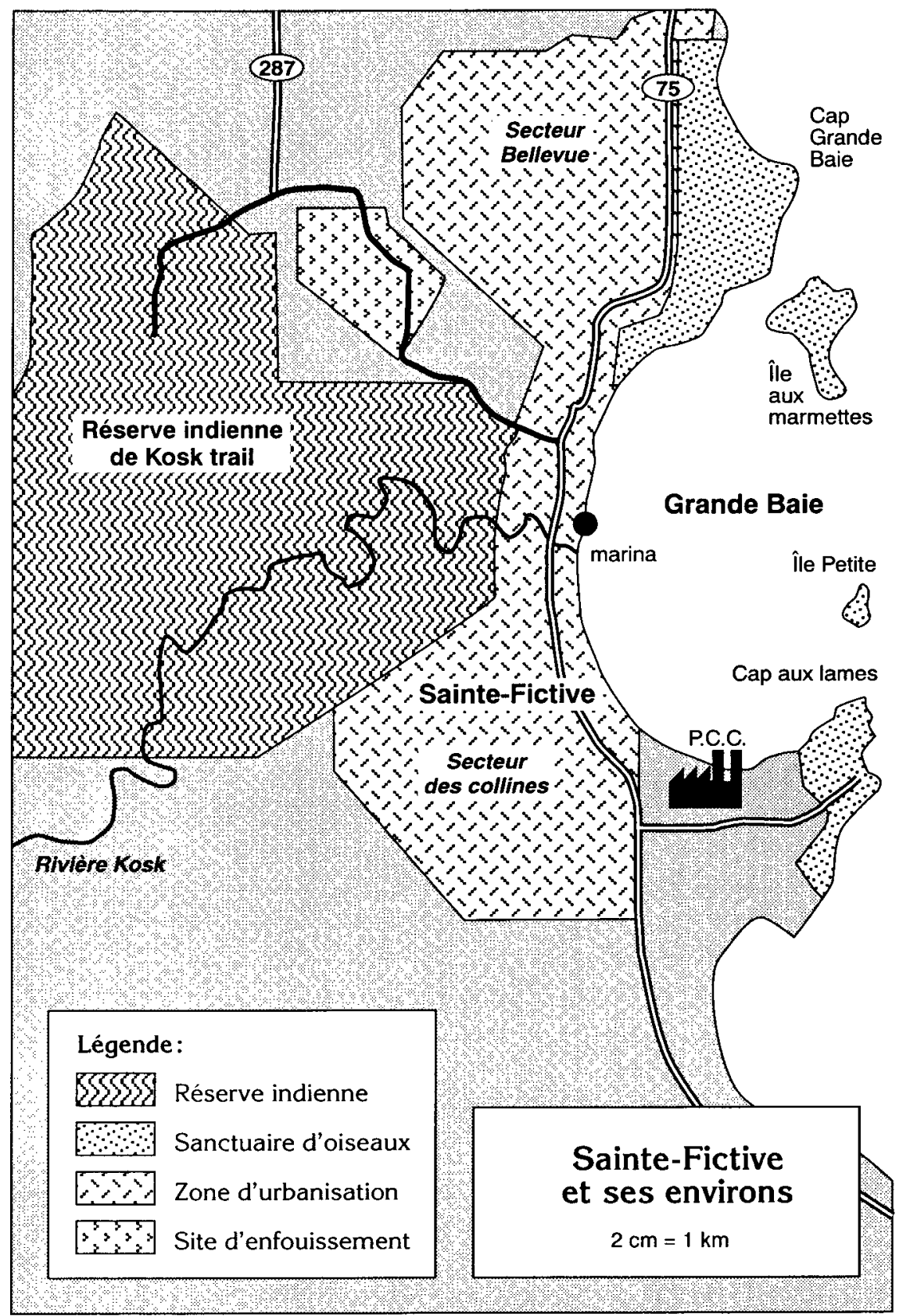

Figure 18: Carte géographique des environs de Sainte-Fictive tirée du jeu de rôle "Alerte à l'environnement " 
Jeu, simulation et jeu de rôle

Voici les groupes représentés :

- une biologiste spécialisée en écologie marine ;

- un représentant des propriétaires de l'usine ;

- le président du syndicat des travailleurs de l'usine ;

- la mairesse de la municipalité ;

- la présidente du regroupement d'artisans locaux ;

- le chef de bande de la réserve indienne ;

- la présidente du comité de citoyens du secteur Bellevue ;

- un travailleur communautaire prenant la défense des citoyens des quartiers défavorisés ;

- une chômeuse à la tête d'un groupe de sans-emploi non subventionné.

La technique employée est celle de l'aquarium. Le groupe-classe est divisé en neuf équipes qui prennent le parti d'un des groupes sociaux. Chacun des rôles énumérés ci-dessus est interprété par un individu alors que les autres membres de l'équipe demeurent à l'écart de la scène. Le jeu de rôle se déroule sur plusieurs jours puisqu'on prend d'abord une période de 50 minutes pour la phase préparatoire puis deux à quatre périodes de plus pour les séances de délibération comme telles. D'une séance à l'autre, un nouvel apprenant est délégué pour tenir le rôle assigné à l'équipe. Soulignons que tous sont activement engagés dans la phase préparatoire des débats.

Ce genre de jeu de rôle offre la possibilité d'approfondir les questions, car les apprenants disposent de temps entre les séances pour étoffer leur argumentation en se documentant et en élaborant ensemble une stratégie. 
Jeu de rôle

La formule des délégués d'équipe qui participent aux délibérations selon la technique de l'aquarium offre deux avantages : une certaine émulation entre les équipes et une plus grande facilité à gérer la séance puisque le nombre d'intervenants est limité à une dizaine à la fois. 
Jeu, simulation et jeu de rôle

\section{« Bafà Bafà »}

par R. Gary Shirts

Type : Jeu de rôle intégrant les caractéristiques du jeu de rôle et de la simulation

Catégorie: Développement d'attitudes

Organisation: Technique centralisée (12 à 35 participants)

Clientèle : Collégial et second cycle du secondaire (il existe aussi une version pour le primaire)

Durée : Environ 2 heures

« Bafà Bafà » est un classique dans le domaine du jeu de rôle. Il vise à développer chez les apprenants une meilleure compréhension des problèmes liés aux relations interculturelles et à leur faire découvrir leurs attitudes vis-à-vis des personnes d'appartenances culturelles différentes.

Deux sociétés dont les valeurs et les comportements sont tout à fait différents sont créées artificiellement (par exemple, une des deux sociétés utilise un langage monosyllabique incompréhensible aux non-initiés). Les participants sont divisés en deux clans et s'exercent d'abord à se comporter selon des règles précises qui ne doivent pas être révélées à ceux de l'autre clan. Ensuite, ils sont envoyés, seuls, deux ou trois à la fois, en visite dans l'autre clan afin d'en étudier le fonctionnement. L'interaction est limitée à quatre ou cinq minutes seulement au cours desquelles les visiteurs tentent de s'intégrer de leur mieux à l'autre culture. De retour « chez eux » les visiteurs relatent leur expérience puis un nouveau contingent de visiteurs est envoyé. Au fil de ces visites et de ces comptes rendus, chaque clan augmente sa compréhension des comportements de l'autre clan et des règles qu'ils suivent. De retour en grand groupe, chacun émet ses hypothèses quant aux règles de l'autre culture qui sont, par la suite, décrites en détail par chacun des clans.

En fait, ce n'est pas durant l'activité elle-même que les participants à " Bafà Bafà » réfléchissent sur les problèmes de relations interculturelles, trop pris qu'ils sont par le déroulement du jeu de rôle. Cependant, la discussion subséquente suscite ce genre de questionnement. 


\section{Chapitre 6 \\ Conception de jeux, simulations et jeux de rôle}

La créativité est une fleur délicate que la confiance fait éclore mais que le doute fane au bourgeon.

Alex F. Osborn

\section{Introduction}

La conception d'un jeu, d'une simulation ou d'un jeu de rôle fait appel à la créativité de l'individu. C'est un processus qui ne saurait être réduit à une série de formules ou de recettes selon une démarche linéaire. La création procède généralement d'une tout autre façon. Par contre, affirmer que la conception d'une activité d'enseignement relève d'une soudaine inspiration et qu'aucune ligne directrice ne peut être fournie au créateur de jeu ou de simulation constituerait une simplification par trop grossière de la réalité.

En fait, il y a lieu d'envisager la conception comme une démarche faite d'intuition, certes, mais aussi d'organisation systématique d'éléments qui contribuent à enclencher un processus de développement assez structuré.

\section{Modèle de conception de jeux, simulations et jeux de rôle}

Afin de guider le concepteur de jeux et simulations, nous proposons un modèle comportant quatre étapes :

- la planification ;

- la scénarisation ;

- l'idéation ;

- la validation. 
Ce modèle est présenté à la figure 19. Voyons maintenant chacune des étapes en détail.

\section{Planification}

(Dans les lignes qui suivent le mot « jeu » désignera autant un jeu, une simulation qu'un jeu de rôle.)

La planification est la première étape et la seule sur laquelle on ne reviendra pas une fois qu'elle sera complétée. On établit ici ses attentes face au jeu ; autrement dit, on détermine un contenu d'apprentissage en relation avec les objectifs pédagogiques visés par le jeu.

- Quelles sont les nouvelles capacités que le jeu fera acquérir ou consolidera?

- Quelles informations doivent être véhiculées ?

- Quels principes le jeu doit-il permettre de dégager?

De plus, on doit définir clairement la fonction que le jeu remplira dans la séquence d'enseignement.

- Le jeu sera-t-il présenté au début d'une session ou d'une leçon pour servir de motivateur, de déclencheur ou bien servira-t-il à effectuer la révision du contenu?

L'étape de la planification est très importante, car en précisant le contenu et la fonction pédagogique du jeu, on se donne un cadre qui facilitera et guidera toute la conceptualisation du jeu. 
Conception de jeux, simulations et jeux de rôle

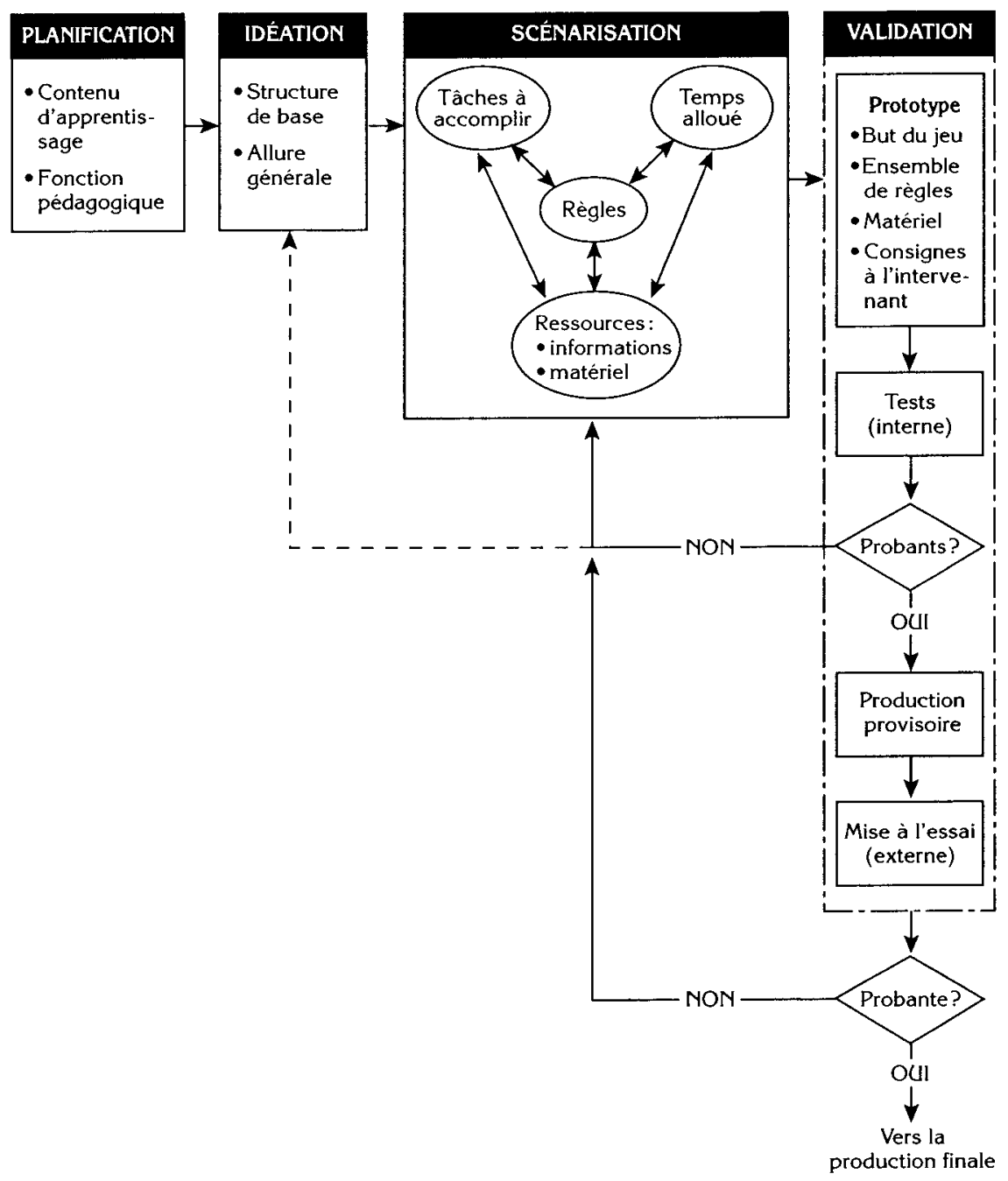

Figure 19: Modèle de conception d'un jeu, d'une simulation ou d'un jeu de rôle 


\section{Idéation}

Il existe un éventail important de modèles pouvant servir à la production d'un jeu. Il s'agit ici d'explorer les différentes structures de base qui pourraient éventuellement servir les fins visées. Il est important de faire une véritable exploration des modèles potentiels avant d'arrêter son choix. Le défaut des concepteurs novices est de se limiter à leur première idée et de ne pas tenter d'en générer un grand nombre, d'en faire l'analyse et d'effectuer un choix.

C'est à cette étape du processus que l'on fait face à cette page blanche si redoutable. L'idée, ou, plus justement, les idées ne surgissent pas nécessairement sur demande On peut alors se poser certaines questions :

- D'où viendront ces idées ?

- Comment faire pour avoir une idée qui soit réalisable ?

Voici quelques étapes que le créateur de jeux peut suivre pour avoir des idées :

- se préparer et placer une commande à son cerveau ;

- laisser incuber ;

- attendre ou provoquer l'illumination ;

- vérifier si cela fonctionne.

Se préparer et placer une commande à son cerveau

Dans un premier temps, le créateur fait un travail de préparation. Il établit, à partir du résultat de sa planification, les contraintes de son projet (contraintes liées à la clientèle visée, la taille $\mathrm{du}$ groupe, l'environnement physique, le temps total dont 
il dispose, etc.) et explore de façon systématique les différents modèles ou pistes applicables. C'est un stade logique.

Il faut semer la graine de l'illumination que nous verrons plus loin. C'est ce qu'on entend par " placer une commande » à son cerveau. On a plus de chances d'avoir des idées si l'on décide qu'on en a besoin et si l'on se place dans un état de réceptivité.

Nos sens filtrent continuellement les informations qu'ils captent et attirent notre attention sur celles qui ont un intérêt pour nous. Vous l'avez sans doute remarqué, lorsqu'on est sur le point d'acheter un certain modèle de voiture, on se met à en voir partout ! Il suffit qu'on apprenne un nouveau mot pour que, tout à coup, on le réentende plusieurs fois dans les semaines qui suivent. Le même phénomène peut être mis à profit pour la recherche de bonnes idées.

\section{Laisser incuber}

Une fois la préparation terminée et la commande placée, l'approche logique est abandonnée, car elle ne produit plus de résultats. Commence alors une période de durée très variable qui est caractérisée par la frustration, le tâtonnement, pour ne pas dire l'errance : c'est le temps de l'incubation.

Il semble alors qu'on ne viendra jamais à bout de la tâche. Pourtant cette étape est profitable, car les éléments apparemment incompatibles ou non pertinents sont soumis à une étude sporadique par l'esprit du concepteur et cela débouchera éventuellement sur un résultat. On peut se consoler en se disant que les meilleurs plats sont ceux qui mijotent longtemps... 
Jeu, simulation et jeu de rôle

\section{Attendre ou provoquer l'illumination}

L'illumination est une inspiration soudaine, une sorte de synthèse instantanée où des éléments disparates s'harmonisent soudainement dans un tout cohérent. Le schéma intégrateur prend donc forme et la pénible période d'incubation prend fin.

L'illumination frappe n'importe où, n'importe quand. Elle vous tirera de votre sommeil en pleine nuit, vous saisira lorsque vous serez sous la douche ou en train de conduire votre voiture. Elle est tout à fait imprévisible, bien que certains moments soient plus propices. Ces moments sont ceux où l'individu vit dans un état second, c'est-à-dire où son esprit n'est pas entièrement sollicité pour réaliser ce qu'il est en train de faire. Quand on exécute une tâche où interviennent des automatismes, des réflexes, et qu'une attention minimale est requise à sa bonne conduite, il est possible de réfléchir ou de laisser son esprit vagabonder quelque peu.

Il vous est peut-être déjà arrivé, par exemple, de conduire une voiture sur une longue distance et d'arriver à destination sans aucun souvenir des événements survenus en cours de route ; votre esprit était occupé à penser à autre chose. Ces occasions semblent être des moments que privilégie l'illumination pour « frapper ».

À la manière du compositeur, de l'artiste peintre ou de l'écrivain, le créateur de jeux peut se placer dans des conditions favorables. Chaque individu a ses moments privilégiés d'inspiration. Certains ont remarqué qu'ils avaient leurs meilleures idées sous la douche, dans leur voiture, au lit, juste avant de s'endormir ou, au contraire, au réveil, voire en pleine nuit. Certaines activités semblent propices à l'idéation: marcher, jogger, jardiner, faire la vaisselle (!), etc. 
Conception de jeux, simulations et jeux de rôle

Les meilleures circonstances semblent avoir ceci en commun qu'elles n'exigent pas du créateur qu'il réfléchisse consciemment à ce qu'il fait. C'est pourquoi les activités machinales que nous avons évoquées prédisposent bien l'individu : l'hémisphère gauche du cerveau, à dominance logique et analytique, est temporairement « engourdi », et c'est l'hémisphère droit, spécialisé dans les tâches globales, l'intuition, etc., qui reprend du service ${ }^{1}$.

En fait, il y a ici une part d'incontrôlable. " Aide-toi, le ciel t'aidera! » Quelles sont les caractéristiques de l'individu créatif ? C'est un individu :

- motivé (fondamentalement désireux d'arriver à un résultat);

- libre (qui n'a pas peur du ridicule et qui évite de s'autocensurer);

- sensible (réceptif à ce qui l'entoure, en éveil, en effervescence).

Quant à l'individu qui mènera à bien son projet, à celui qui saura traduire sa créativité en un résultat concret, c'est essentiellement celui qui ajoutera, comme le disait Henry Ford, « $95 \%$ de transpiration au $5 \%$ d'inspiration » qu'il aura eue.

\section{Vérifier si ça fonctionne}

L'illumination est associée à une certaine euphorie. Il faut éventuellement reprendre contact avec la réalité et soumettre cet " éclair de génie » à un examen attentif afin d'en évaluer la faisabilité.

1. Les études sur les fonctions intellectuelles et la manière dont elles se répartissent dans les lobes droit et gauche du cerveau humain démontrent une spécialisation de l'hémisphère gauche pour les tâches d'analyse, le raisonnement logique, le rationnel, la symbolique, le langage et la pensée abstraite ; à l'hémisphère droit on associe les tâches de synthèse, l'intuition, l'irrationnel, l'appréhension globale, les relations analogiques et le domaine du concret (Levy, 1968 ; Sperry, 1973). 
Jeu, simulation et jeu de rôle

La vérification constitue donc un retour au stade logique où sont plus froidement étudiées les découvertes secondaires et les inspirations qu'on a eues « à chaud ». Cette étape préfigure le travail de scénarisation qui suit immédiatement l'idéation du modèle.

$\mathrm{Si}$, comme bien des gens, vous entretenez quelque doute sur votre capacité de concevoir des jeux ou simulations, considérez ce qui suit. Sur une période de douze ans, dans le cadre d'un cours universitaire de premier cycle, nous avons demandé aux enseignants ou futurs enseignants de créer un jeu, une simulation ou un jeu de rôle. Sur les mille et plus auxquels nous avons enseigné, un grand nombre craignaient de ne pas avoir d'idée. Pourtant, pratiquement tous ont réussi à créer leur jeu ; certains ont même conçu des jeux qui ont été largement diffusés.

Nous suggérons donc à l'enseignant qui désire concevoir un jeu de relire l'épigraphe du début de ce chapitre, de planifier placer une commande à l'hémisphère droit de son cerveau et de s'accorder quelques jours pour que l'idée surgisse.

\section{Scénarisation}

Une fois le concept de base arrêté et l'allure générale décidée, il faut l'incarner dans une scénarisation. L'idée retenue est développée en fonction des principaux éléments du jeu. On essaie de voir comment concrètement l'idée retenue pourrait prendre forme.

Voyons d'abord les principaux éléments dont on doit tenir compte dans le processus de développement d'un jeu :

- la tâche ;

- les ressources disponibles;

- le facteur temps ;

- les règles. 
La tâche est l'élément de départ.

- Qu'est-ce qui est attendu du joueur?

- Comment agit-il dans le jeu?

Le temps alloué est une contrainte très importante à considérer lors de la scénarisation, à plus forte raison dans un contexte scolaire. La durée prescrite pour un cours, la proportion de temps qu'il est raisonnable d'utiliser pour les objectifs poursuivis compte tenu des autres activités à réaliser dans le cours sont autant de limites dont il faut tenir compte. Certains jeux sont très précis en ce qui concerne la gestion du temps, alors que d'autres laissent pleine latitude aux joueurs à cet égard; le facteur temps ne doit cependant jamais être sous-estimé, car c'est un élément fondamental du succès que le jeu connaîtra auprès des joueurs (motivation).

Les ressources dont les participants disposeront doivent ensuite être définies. Ces ressources sont de deux ordres: des informations et du matériel. Tout jeu suppose une circulation, un échange quelconque d'informations ou de matériel qui est le prétexte et l'objet des interactions entre les joueurs. La teneur pédagogique est souvent étroitement liée à la nature de ces informations ou aux habiletés nécessaires pour transiger le matériel. La quantité d'information, soit le nombre de cartes, de pièces, d'objets à manipuler ou à transiger, etc., doit être calculée et ajustée afin de permettre un usage agréable et efficace du jeu. C'est sur ce point que nous relevons le plus fréquemment des faiblesses, et ce, même dans le matériel commercial.

$\mathrm{Au}$ centre de cette mise en place des éléments constitutifs du jeu, les règles fournissent le cadre, la structure, la colonne vertébrale de la scénarisation. En fait, elles conditionnent les trois autres aspects. Elles sont les balises pour la tâche. Elles 
stipulent comment le temps disponible sera utilisé et déterminent quel usage les joueurs feront des ressources. Les règles sont le ciment des divers éléments du jeu.

Étant donné leur haut niveau d'interrelations, ces quatre éléments, tâche, temps, ressources et règles, doivent être traités simultanément et non de manière cloisonnée. Les choix faits au regard de la tâche auront nécessairement une influence sur le temps et les ressources, et vice versa.

La scénarisation est en fait une première mise en forme de l'idée retenue à l'étape de l'idéation, et il est possible qu'elle fasse l'objet d'une remise en question et de fréquentes modifications. Ces ajustements sont normaux et font partie de la dynamique nécessaire à l'arrimage de la théorie à la pratique.

\section{Validation}

La validation est l'étape qui révélera si les choix effectués antérieurement sont pertinents et si le jeu fonctionne bien. La première chose à faire est de produire un prototype. Non seulement nécessaire à la validation, il permet aussi de donner une idée concrète du jeu et des problèmes techniques de production future.

Le prototype est une esquisse complète et détaillée du matériel qui, sous une forme souvent temporaire, doit être rassemblé. Un premier jet du livret d'instructions ou autre matériel écrit qui présente le jeu doit être réalisé. Tous les aspects de la réglementation doivent y apparaître. On rédige aussi les consignes destinées à l'intervenant en ayant en tête la logistique du jeu et son exploitation pédagogique.

Le prototype doit ressembler le plus possible au produit fini qu'on envisage de produire. On utilisera vraisemblablement 
des matériaux de qualité inférieure (comme des cartes moins rigides, par exemple), mais il est préférable de respecter les dimensions prévues pour chaque pièce de matériel, la taille du lettrage, etc. Le prototype devrait être produit en couleurs si celles-ci ont une importance dans le jeu.

Une fois le prototype réalisé, on passe au premier niveau de validation comme tel: les tests réalisés à l'interne, c'est-à-dire par soi-même ou par quelques collaborateurs. Cette validation consiste en une ronde « à blanc » ou en une première utilisation du jeu pour en évaluer la mécanique. Concrètement, il s'agit de procéder exactement comme s'il s'agissait du déroulement véritable du jeu sauf que le concepteur joue seul ou est aidé de quelques « cobayes ».

Il est certain que les conditions réelles du jeu ne peuvent souvent pas être reproduites à cette étape. Par exemple, un jeu conçu pour être joué à 35 personnes sera peut-être validé avec 5 à 10 participants et il sera alors nécessaire d'extrapoler pour évaluer le bon déroulement du jeu avec le nombre de joueurs prévus. L'élément clé des tests est le bon jugement du concepteur.

Les tests sont menés en vue de répondre à la question suivante: le jeu fonctionne-t-il ? Ici, fonctionner veut tout aussi bien dire « se dérouler sans anicroches que produire les résultats désirés », à savoir favoriser l'atteinte des objectifs visés. Notons qu'il est parfois difficile d'évaluer à court terme l'atteinte des objectifs, ce qui nous amène, dans bien des cas, à nous limiter à une vérification des seuls aspects mécaniques du jeu.

Si les tests sont probants, c'est-à-dire si le jeu fonctionne bien, on passe à l'étape suivante. Si les tests ne sont pas probants (ce qui est tout à fait habituel), c'est-à-dire s'ils révèlent des 
difficultés de fonctionnement, il faut revoir le processus de développement. La plupart du temps, une foule de petits détails doivent être changés à cette étape, de même que certains aspects de la scénarisation. Plus rarement, on reviendra même plus loin en arrière et l'on remettra en question les choix faits à l'étape de l'idéation.

Le cycle «modifications à la scénarisation - ajustement du prototype - tests » peut se répéter quelques fois, car il est rare que le jeu fonctionne du premier coup. Ultimement, cependant, les tests finiront par être probants et l'on pourra procéder à la production du matériel sous une forme provisoire.

On fabrique alors le matériel de jeu avec des matériaux de première qualité et on rédige les documents au propre. Dans un processus de copie en grand nombre, on en serait à la copie zéro.

Le jeu est maintenant fin prêt pour un deuxième type de validation: la mise à l'essai auprès de la clientèle visée. Contrairement à ce qui est fait lors des tests, la mise à l'essai se déroule dans les mêmes conditions que celles prétablies par le concepteur: les joueurs sont présents en nombre suffisant et le matériel est présenté exactement dans la forme prévue pour la diffusion ultérieure du jeu.

Puis, on joue. Le concepteur intervient le moins possible durant le jeu ; il observe et prend note de tout détail pertinent. Idéalement, le jeu est présenté et animé (le cas échéant) par une tierce personne, permettant ainsi au concepteur d'évaluer la facilité avec laquelle l'utilisateur potentiel, le professeur ou le formateur, procède, de même que son degré de compréhension des consignes préparées à son intention. 
Quand toutes les étapes préalables sont respectées, il est rare que la mise à l'essai se solde par un échec retentissant. Le plus souvent, il s'agit d'ajustements mineurs. Pour le concepteur, c'est probablement la dernière occasion de revenir à l'étape de la scénarisation.

\section{Jeu-cadre}

La démarche de conception que nous venons de présenter est sans doute trop lourde pour être appliquée chaque fois que l'on veut bâtir un jeu ou une simulation.

Par ailleurs, les jeux et simulations qui existent déjà et dont peut disposer l'enseignant ou le formateur ne peuvent évidemment pas cadrer avec tous les objectifs et couvrir tous les contenus.

Le problème qui se pose est donc celui-ci : l'enseignant se trouve coincé entre l'option d'utiliser les jeux et simulations disponibles (limités dans leur nombre et les contenus qu'ils touchent) et la perspective de devoir en créer de toutes pièces avec tout ce que cela comporte d'investissement de temps et d'énergie.

C'est ici qu'intervient le jeu-cadre, car il peut contribuer à résoudre efficacement ce problème par sa souplesse et sa simplicité. C'est un jeu que l'on modifie afin de créer un nouveau jeu. C'est donc au départ un jeu caractérisé par une " prédisposition aux changements » et dont l'efficacité de la structure est acquise (Stolovitch et Dupont, 1979).

Pour qui souhaite bâtir un jeu sans partir de zéro, cela revient à s'appuyer sur un jeu existant, à l'adapter, à le transformer, afin qu'il convienne aux objectifs d'apprentissage prédéter- 
minés. Le résultat est un nouveau jeu efficient et efficace. Efficient, car le contenu pédagogique est bien couvert après avoir apporté certaines modifications au jeu-cadre. Efficace, car ces modifications n’ont pas altéré la qualité de fonctionnement du jeu-cadre.

\section{Structure et contenu : deux éléments fondamentaux d'un jeu}

Tout jeu peut être divisé en deux éléments de base : la structure et le contenu. La structure détermine la manière de jouer : ce sont les règles, les phases ou étapes de déroulement, le défi que les joueurs doivent relever et les stratégies qu'ils peuvent déployer pour gagner. Le contenu renvoie aux informations véhiculées dans le jeu : dans le cas des jeux à caractère pédagogique, il s'agit aussi des objectifs poursuivis et des habiletés qui seront développées par la pratique du jeu.

On reconnaît un bon jeu à l'harmonie qui lie la structure et le contenu; le jeu-cadre répond à cette exigence. Mais ce qui le rend particulièrement pratique, c'est que d'autres contenus peuvent se substituer au contenu original tout en étant parfaitement compatibles avec la structure. C'est cette caractéristique fondamentale, l'interchangeabilité des contenus, qui fait du jeu-cadre un outil pédagogique aussi intéressant.

La figure 20 illustre le concept d'interchangeabilité du contenu.

Ainsi, un jeu-cadre peut généralement servir un grand nombre de fins pédagogiques différentes. À partir du même jeu-cadre, on peut construire autant de nouveaux jeux que de nouveaux contenus compatibles. 


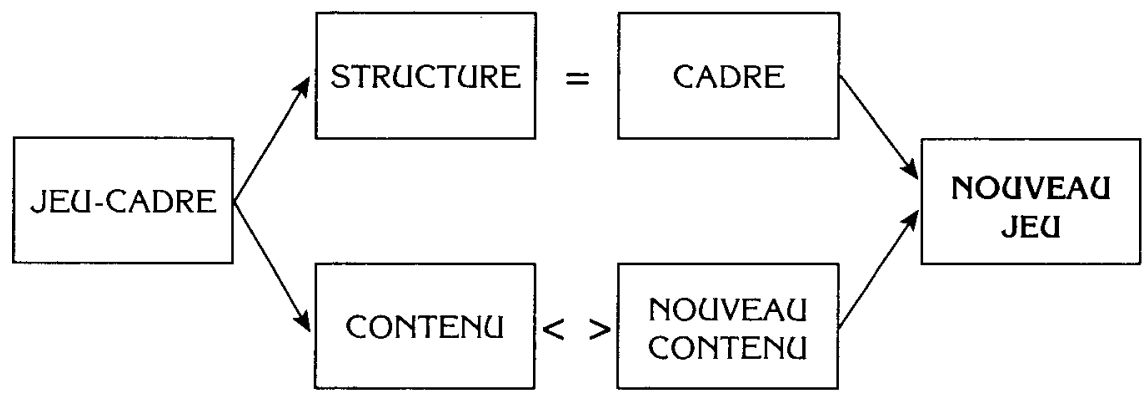

Figure 20: Composantes du jeu-cadre (adapté de Stolovitch, 1989)

Considérons, à titre d'exemple, un jeu-cadre bien connu de tous, le bingo. Dans la version originale du jeu, on doit former sur une matrice de cinq cases sur cinq une suite de cinq jetons alignés verticalement, horizontalement ou diagonalement; on reconnait ici la structure du jeu qui deviendra notre cadre. Les jetons sont placés sur les nombres apparaissant dans les cases à mesure qu'ils sont nommés par le meneur de jeu; les nombres de 1 à 75 constituent le contenu.

En conservant la matrice de cinq cases sur cinq et en rem- plaçant les nombres par des illustrations de panneaux routiers, on obtient un nouveau jeu, en l'occurrence le jeu « Sagix » produit par le ministère des Transports du Québec (voir figure 21).

On reconnaîtra, bien sûr, dans ce nouveau jeu la structure de l'ancien. On a même conservé la case gratuite du centre où apparaît maintenant le pictogramme du port obligatoire de la ceinture de sécurité. Ce qui est nouveau dans ce jeu, c'est le contenu. En ayant à repérer sur sa carte différents pictogrammes, l'enfant apprend à reconnaître leur configuration et éventuellement à décoder leur signification. 


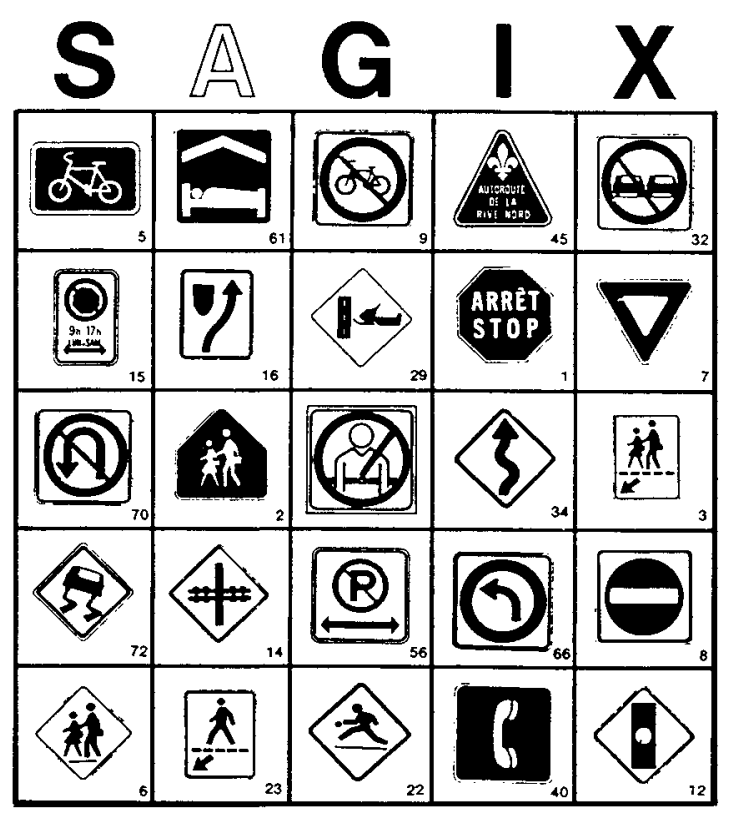

Figure 21: Exemple d'application du bingo comme jeu-cadre: "Sagix"

\section{Plusieurs types et niveaux d'adaptation}

Une première façon de jouer le jeu « Sagix » consiste à montrer le pictogramme pigé en le tenant bien à la vue des élèves sans mentionner sa signification. Il s'agit pour le joueur de repérer parmi les pictogrammes de sa carte celui que le meneur de jeu présente.

« Sagix » peut également se jouer d'une manière quelque peu différente. Plutôt que de montrer le pictogramme, le maître peut dire à haute voix sa signification, laissant à l'élève le soin d'y associer le pictogramme correspondant. Ainsi, Accès interdit aux bicyclettes donne le droit au joueur de placer un jeton sur la première case de la colonne $« \mathrm{G} »$ dans notre exemple. 
Cette seconde façon d'exploiter le même jeu n'a de nouveau rien modifié à la structure du jeu de bingo ; il s'agit d'une adaptation qui touche essentiellement le contenu pour lequel on a changé le niveau de difficulté.

Dans certains cas, cependant, certaines modifications peuvent toucher la structure du jeu-cadre; voilà un second type d'adaptation. Considérons, pour illustrer cela, un nouvel exemple.

Le jeu de tic-tac-toe, connu de tous, est un autre jeu-cadre typique. Le jeu « Ça va vous tuer » est tiré du tic-tac-toe (voir figure 22). Tout comme pour le jeu "Sagix », on a complètement changé le contenu de l'ancien jeu pour lui en substituer un nouveau. Cependant, dans " Ça va vous tuer », la structure du jeu, le cadre, a également subi des transformations.

Voyons ce qui, dans la structure, demeure inchangé et ce qui a été modifié.

D'abord les deux jeux se jouent sur une matrice. Pour le tic-tac-toe, elle comprend trois cases sur trois; Ça va vous tuer nécessite une matrice de quatre cases sur six.

Le but du jeu est le même dans les deux cas : aligner un certain nombre de symboles identiques verticalement, horizontalement ou diagonalement. Pour le tic-tac-toe, ce nombre est de trois ; pour « Ça va vous tuer ", il est de quatre. Le tic-tac-toe permet à deux adversaires de s'affronter; Ça va vous tuer permet à deux ou trois équipes de le faire. 
Jeu, simulation et jeu de rôle

\section{ÇA VA VOUS TUER}

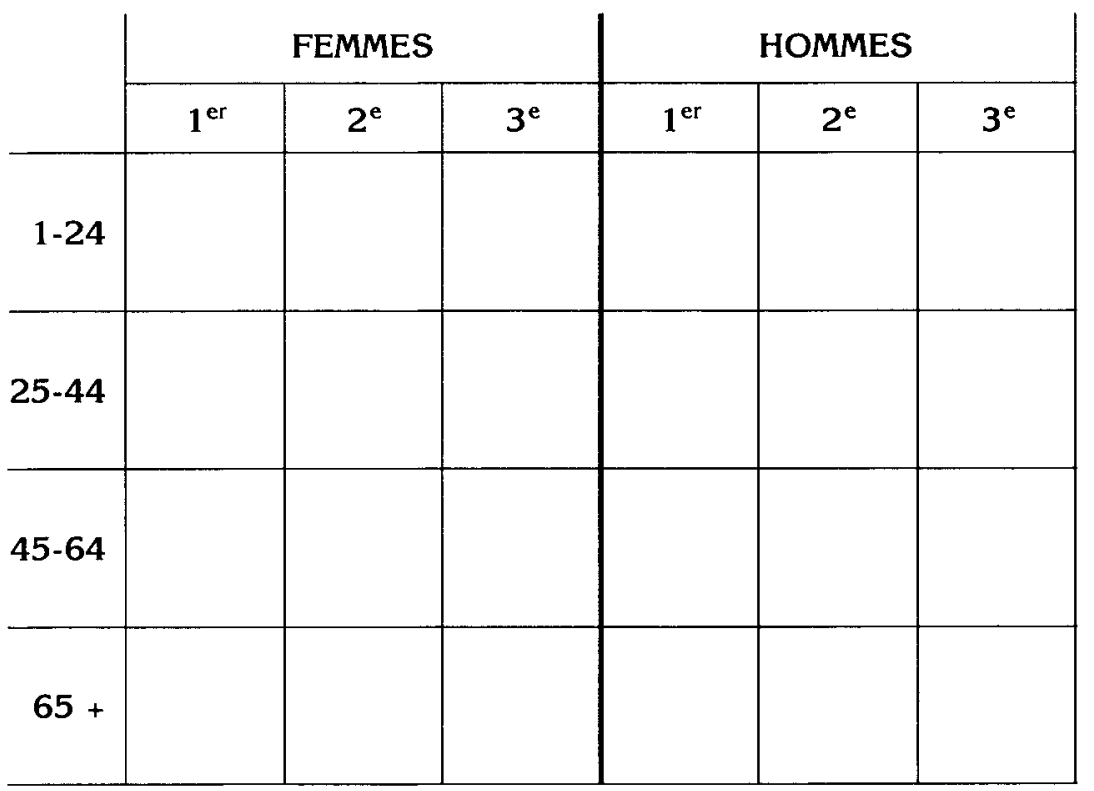
A. Accidents
B. Anomalies congénitales
C. Cancer
D. Maladies cardiovasculaires
E. Maladies du système nerveux
F. Maladies endocrines, nutrition et métabolisme
G. Maladies respiratoires
H. Maladies du système digestif

Figure 22: Exemple d'application du tic-tac-toe comme jeu-cadre: "Ça va vous tuer" (adapté de Stolovitch et Thiagarajan, 1980) 
Toutes ces différences ont trait à la structure. Quant au contenu, il est bien sûr différent dans les deux jeux. Les $\mathrm{X}$ et les $\mathrm{O}$ ont fait place à d'autres symboles (carrés, triangles et cercles, par exemple) à l'intérieur desquels les lettres $\mathrm{A}, \mathrm{B}, \mathrm{C} . . \mathrm{H}$ apparaissent, ces lettres étant en relation avec la première, la deuxième ou la troisième cause de mortalité des femmes et des hommes de quatre groupes d'âge au Canada. La cause de mortalité choisie par les joueurs d'une équipe doit convenir à la case où ils désirent mettre leur symbole à défaut de quoi ce sera le tour de la prochaine équipe de jouer. Par contre, au tic-tac-toe chaque joueur, à son tour, place son symbole simplement là où il le désire.

\section{À éviter avec les jeux-cadres}

L'emploi de toute formule pédagogique exige du discernement et le jeu-cadre n'échappe pas à cette règle. Malgré les avantages dont nous venons de traiter, le jeu-cadre n'est pas la panacée et il serait illusoire d'espérer y recourir en toute situation.

Le premier piège à éviter est donc celui des «mariages forcés 》 où l'on tente de faire d'un jeu-cadre le véhicule d'un contenu qui s'y prête mal ou pas du tout. Le second piège consiste à surutiliser le même jeu-cadre, par exemple le bingo. Il est vrai que plusieurs de ces jeux conviennent bien à une foule de contenus différents, mais recourir à outrance à ces jeux a pour effet de miner l'enthousiasme des participants; l'utilisation routinière de cette formule est finalement tout aussi ennuyeuse que n'importe quelle autre routine.

Le jeu-cadre est un outil fort pratique pour réaliser des jeux qui fonctionnent bien et qui touchent les contenus pertinents aux objectifs pédagogiques que poursuit l'enseignant ou le formateur. 
Le recours à cette formule est souvent une façon heureuse de s'épargner la création de jeux complètement originaux ou de remplacer des jeux existants qui suffisent rarement à couvrir l'ensemble des contenus que l'on souhaiterait aborder par le jeu.

Moyennant quelques réaménagements dans la structure pour satisfaire les exigences du nouveau contenu, l'enseignant dispose d'un matériel adapté à ses besoins, donc efficient, et d'un fonctionnement éprouvé, donc efficace.

\section{Quelques conseils pratiques pour la production d'un jeu à tablier}

La production de jeux à tablier présente certains problèmes particuliers qu'on ne retrouve pas, par exemple, dans la production des jeux de rôle. En effet, ces derniers prenant généralement la forme de textes sur des cartons ou des feuilles de papier, une mise en page efficace suffira à leur donner une allure correcte. Dans le cas des jeux à tablier, ce sont vos talents en graphisme et en bricolage qui seront mis à contribution.

La production d'un jeu à tablier nécessite un investissement de temps et d'argent. Comme un jeu éducatif doit être aussi intéressant et agréable à jouer qu'un jeu de société, il est très important que sa présentation physique se conforme le plus possible aux standards de la majorité des jeux à tablier commerciaux. L'aspect général du jeu doit être conçu et produit pour capter l'attention et susciter l'intérêt.

La production définitive du tablier ne doit s'effectuer qu'après la validation à l'aide du prototype. Un plan complet et précis doit donc être réalisé avant le début de la production. L'excitation et l'enthousiasme qu'engendre l'entreprise de créer son 
propre jeu amènent très souvent le néophyte à se lancer dans la réalisation définitive du tablier avant même d'en avoir terminé le plan complet. Plusieurs frustrations peuvent être évitées, avec les dépenses en temps et en argent que cela occasionne, si le créateur sait réprimer son envie de sauter l'étape de planification détaillée qui est, en fait, la manière la plus rapide d'arriver à un résultat satisfaisant.

Le tablier est la pièce principale et celle qui, en un clin d'œil, révèle tout ce qu'est votre jeu. Il ne faut surtout pas s'arrêter à la première idée qui vous vient. Le tablier doit être le résultat d'un choix fait parmi de nombreuses possibilités. La production d'un jeu demeurera toujours une réalisation où la créativité et l'imaginaire de l'auteur doivent avoir été exploités à fond. Bien qu'il s'agisse d'une forme très courante, il est possible de faire de son jeu à tablier une œuvre unique et originale. Cependant, il ne faut pas oublier que l'objectif premier est l'apprentissage. En ce sens, moins d'originalité et plus d'efficacité sont toujours préférables à l'inverse.

Un carton de bonne qualité est recommandé pour produire le tablier. Comme il sera l'objet de nombreuses manipulations et qu'il devra pouvoir absorber la vigueur de certains gestes d'enthousiasme que ne manquera pas d'engendrer votre jeu, un matériel rigide et résistant est essentiel (épaisseur minimum recommandée : 14 plis).

Habituellement un carton de couleur blanche ou pâle convient mieux, car il permet de faire ressortir les différentes figures et les dessins qui apparaîtront sur votre tablier.

Attention! Il ne faut pas créer un tablier aux dimensions qui le rendent peu commode à ranger ou à manipuler. Ce facteur peut avoir un effet direct sur l'utilisation de votre jeu. Le carton, qui dans la plupart des cas est de forme carrée ou rectangulaire, se plie habituellement par le centre. Pour qu'il s'ouvre et se 
ferme facilement, il faut le couper à demi ou complètement et le lier avec une bande de papier ou de coton collant. Lorsque plié, le jeu doit se trouver sur la face intérieure de manière à être protégé. Au moment de décider de la position des cases, il est bon de tenir compte de l'endroit prévu pour le pli afin d'éviter qu'une case le chevauche, car une pièce tiendra moins bien sur le pli.

Seuls les meilleurs matériaux accessibles au créateur doivent être utilisés pour produire les formes, les mots, les chiffres et les dessins devant être placés sur le tablier. Une bonne règle, d'excellentes plumes feutres à encre indélébile et peut-être un pochoir ou deux peuvent suffire à celui qui n'a pas accès à du matériel plus perfectionné. Les logiciels graphiques les plus communs sont très utiles dans la réalisation de plusieurs aspects de la production.

Pour lui assurer une certaine durabilité et une certaine protection antitache, la plastification est fortement recommandée. Pour quelques dollars, vous donnerez à votre jeu un fini de qualité tout en le préservant des nombreux risques inhérents à un usage répété.

La confection des pièces à manipuler sur le tablier pose, quant à elle, des problèmes particuliers. Fabriquer des pièces en trois dimensions peut s'avérer fastidieux ; une certaine sobriété est donc conseillée. Certains magasins spécialisés dans les jeux ou le matériel de papeterie vendent des jetons, dés et autres accessoires que l'on retrouve dans la plupart des jeux. Les acheter tout faits constitue une option intéressante. Si vous décidez tout de même de les confectionner vous-même, tenez compte des critères suivants : 
- poids (une pièce trop légère n'est pas agréable à manipuler et risque de se déplacer inopinément) ;

- stabilité ;

- résistance aux chocs et à la manipulation ;

- uniformité (quand un même joueur a plus d'une pièce) ;

- unicité (on doit pouvoir distinguer facilement les pièces d'un joueur de celles d'un autre, donc couleurs ou formes variées).

Le jeu doit se ranger facilement. Tout doit entrer dans une seule boîte de dimensions raisonnables et d'une rigidité lui permettant de résister aux manipulations que suppose un usage normal. La boîte devrait être construite de façon à limiter le mouvement des pièces et autres accessoires qu'elle renferme. Enfin, elle doit être attrayante, ou du moins doit-elle porter le titre du jeu afin qu'on ne l'utilise pas pour autre chose, laissant le tablier et les pièces du jeu sans emballage.

Un livret d'instructions est un élément essentiel du jeu lorsque celui-ci est conçu pour être utilisé de façon autonome par les joueurs. Il comprendra une liste du matériel (afin de pouvoir vérifier s'il manque des éléments), une brève description du jeu (pour donner une idée générale au néophyte), une énumération de détails pratiques (âge cible et nombre de joueurs, temps requis pour une partie, etc.) et une description claire et concise des règles du jeu. Le but du jeu devrait être explicite. Il faut éliminer tout élément non essentiel et réduire le texte à sa plus simple expression ; le style télégraphique peut même être utilisé là où cela ne nuit pas à la compréhension. 



\section{Chapitre 7}

\section{Préparation, animation et exploitation des simulations et des jeux de rôle}

La chance favorise l'esprit préparé.

Pasteur

\section{Introduction}

Le succès d'une simulation ou d'un jeu de rôle et la qualité de ce qu'on en retire sont également liés à la compétence de la personne qui l'anime et qui dirige la discussion subséquente. Bien que le succès d'autres formules pédagogiques repose sur les qualités de l'intervenant, il convient de souligner que ce facteur revêt une importance particulière dans le cas de la simulation et du jeu de rôle.

Les activités reliées à ces deux formules se présentent sous une grande variété de formes et de styles. Lorsque vient le moment de les utiliser dans le contexte de l'enseignement, il n'est pas possible d'appliquer la même procédure, de prévoir les mêmes conditions matérielles, ni le même investissement de temps. Chaque situation est un cas d'espèce, et l'arrimage avec les autres activités d'apprentissage doit être planifié en tenant compte de la particularité de la situation.

\section{Préparation}

La plupart des problèmes rencontrés lors des jeux de rôle ou des simulations ne sont généralement pas imputables à la valeur du jeu de rôle ou de la simulation, mais plutôt au 
mauvais usage qui en est fait. Dans le même ordre d'idées, un mauvais usage découle généralement d'une préparation insuffisante. Nous entendons par préparation tout ce que l'intervenant doit faire avant que l'activité proprement dite démarre. Nous distinguons plus précisément les deux phases suivantes :

- la planification (plusieurs jours avant l'événement) ;

- les préparatifs (quelques heures ou minutes avant l'événement).

\section{Planification}

Supposons que l'enseignant a déjà sélectionné un jeu de rôle ou une simulation qui convient à certains objectifs pédagogiques qu'il poursuit. Il lui faut d'abord situer cette activité dans la séquence d'enseignement en tenant compte de la fonction qu'il veut lui attribuer: s'agit-il de déclencher l'intérêt ou plutôt de reprendre et de synthétiser les éléments de théorie déjà présentés ?

Une bonne planification comporte habituellement les étapes suivantes :

- Identifier les autres activités d'enseignement périphériques à l'utilisation du jeu de rôle ou de la simulation et les ordonner de façon à constituer une séquence d'enseignement logique et bien articulée.

- Prévoir les liens nécessaires entre ces diverses activités didactiques (le jeu de rôle ou la simulation ne doit pas arriver comme un cheveu sur la soupe et rompre la continuité des événements mais plutôt s'y intégrer harmonieusement).

- Jouer le jeu de rôle avant (ou l'étudier soigneusement) afin d'acquérir une excellente connaissance de son déroulement, ce qui est essentiel ; c'est le meilleur gage de succès. 
Préparation, animation et exploitation des simulations et des jeux de rôle

Cette étape s'apparente à ce que nous appelions les tests internes à l'étape de validation au chapitre précédent. Le temps investi à procéder à cette opération vaut son pesant d'or ; en plus d'être une sorte de répétition pour celui qui sera appelé à animer le jeu de rôle, c'est une occasion pour lui de repérer les problèmes potentiels et d'imaginer les solutions pour y remédier.

Afin d'assurer un bénéfice maximal pour la clientèle visée, modifier l'activité au besoin sera donc la prochaine tâche de planification à réaliser. Au-delà des ajustements nécessaires en relation avec cette clientèle, il faut aussi prévoir un rôle, une fonction pour chacun des individus du groupe. Il est important que tous participent et, pour ce faire, plusieurs options sont possibles pour résoudre un problème courant, à savoir l'écart entre le nombre de joueurs prévu aux règles et le nombre de personnes qui composent le groupe :

- attribuer chacun des rôles à autant d'équipes formées de deux ou trois individus ;

- faire participer les personnes à tour de rôle plutôt que toutes ensemble ;

- former des sous-groupes qui reproduiront le même scénario simultanément ;

- créer de nouveaux rôles et les intégrer à l'action, lorsque cela est possible.

Le choix de la formule d'adaptation est laissé au jugement et à la discrétion de l'animateur qui sait le mieux évaluer les contraintes liées à la gestion de l'activité.

La dernière étape de la planification consiste à organiser les ressources humaines et matérielles : contacter les gens dont on veut s'assurer la collaboration, réserver le matériel et les locaux, commander le matériel nécessaire, etc. 
Concernant le lieu où se tiendra l'activité, il est important de noter que jouer ou simuler suppose la plupart du temps un besoin d'espace beaucoup plus grand que pour donner un cours magistral ; un groupe de 25 personnes participant à une simulation aura besoin d'un local prévu pour 50 ou plus pour avoir la liberté de mouvement nécessaire. La possibilité de déplacer le mobilier est également à considérer.

\section{Préparatifs}

Lorsque toutes les étapes de la planification ont été franchies, quelques heures avant la tenue du jeu de rôle ou de la simulation, on doit procéder aux derniers préparatifs. Le compte à rebours est enclenché et rien ne doit être négligé pour « l'heure $\mathrm{H}$ ».

Il s'agit tout d'abord de vérifier si toutes les conditions à réunir pour l'événement seront effectivement en place - avoir confirmation de la présence des collaborateurs, s'assurer que la salle sera libérée, que le mobilier y sera placé comme prévu, etc.

L'environnement physique revêt une très grande importance dans la plupart des simulations et des jeux de rôle. La configuration des lieux influence les interactions entre les participants plus qu'on n'aurait tendance à le croire. À titre d'exemple, les personnes qui occupent le centre de la pièce dans une simulation qui encourage peu le mouvement sont nécessairement en contact avec un plus grand nombre d'individus que celles qui se trouvent dans les coins ou sur le pourtour de l'aire de jeu. Il faut donc aménager les lieux de manière à contribuer à la fluidité des interactions. Malheureusement, l'accès au local n'est souvent possible que quelques minutes avant le début de la rencontre ; il est alors 
Préparation, animation et exploitation des simulations et des jeux de rôle

primordial de n'avoir plus que cela à faire afin de ne pas s'exposer à des délais supplémentaires.

Préparer tout le matériel et l'agencer de manière à faciliter sa présentation ou sa distribution constitue un autre élément clé des préparatifs. En réalisant cette tâche suffisamment longtemps à l'avance, l'animateur évitera des moments de stress fort désagréables et il s'assurera de pouvoir se concentrer sur ce qu'il a à faire au moment où les participants arriveront. Il est recommandé de procéder à un inventaire final du matériel après les manipulations et de ranger le tout en lieu sûr.

Une gestion efficace du temps est une autre pierre angulaire du bon déroulement du jeu de rôle. Il ne faut donc pas négliger de constituer un horaire le plus précis possible. Des balises de temps sous forme de fourchettes conviennent dans la majorité des cas (exemple : étape $\mathrm{X}, 15$ à 20 minutes; étape $\mathrm{Y}, 10$ à 12 minutes, etc.).

Ne pas oublier de prévoir du temps pour le debriefing dont nous traiterons plus loin. Bien entendu, le cumul de tous les maximums (dans notre exemple 20 minutes plus 12 minutes, etc.) ne doit pas excéder la période de temps totale dont on dispose.

L'horaire peut être complété de courtes notes indiquant les principales opérations et les remarques à communiquer aux participants ; dresser un tel aide-mémoire est essentiel tant que l'animateur n'a pas une parfaite maîtrise de l'activité, ce qui ne s'acquiert qu'après plusieurs expériences. Ce petit guide se voudra succinct, écrit en style télégraphique et la mise en page devra refléter la structure de l'activité. Un tel outil doit être mis à la disposition de toute autre personne agissant comme assistant ou ayant un rôle particulier à assumer. 
Ensuite, l'animateur aura avantage à intérioriser les étapes principales afin d'avoir en tête un schéma global de l'activité ; cela lui évitera d'errer ou de s'éloigner du but poursuivi.

Toute tâche ou manipulation que l'animateur devra effectuer dans le feu de l'action doit être maîtrisée par lui. Il lui faut donc pratiquer les manipulations de façon à les automatiser pour éviter qu'elles ne ralentissent le déroulement de l'action.

Enfin, il n'est pas rare qu'une certaine nervosité accompagne le début de la rencontre ; c'est pourquoi il est parfois utile de composer un court texte de lancement de l'activité destiné à mettre de l'ambiance et à donner le ton. En fait, il s'agit de se trouver une façon de démarrer.

À la fin du présent chapitre, nous reprenons de façon systématique chacun des éléments relatifs à la préparation sous forme de liste de vérification.

\section{Animation}

Étymologiquement, animer signifie donner une âme L'animateur est donc celui qui donne vie au jeu de rôle ou à la simulation. Pour ce faire, il doit communiquer son enthousiasme et s'assurer le concours des participants. Une bonne animation commence donc par un certain sentiment d'excitation que vit l'animateur et qu'il voudra contagieuse. Pour arriver à transmettre cet état d'esprit et à le canaliser pour le bon déroulement de l'activité, l'animateur doit cependant trouver le juste équilibre sur plusieurs plans.

Cette partie du chapitre est largement inspirée des articles de Thiagarajan (1977) et de Bjur (1977). Nous tenterons de guider le lecteur dans cette tâche qui exige de la sagacité. 
Préparation, animation et exploitation des simulations et des jeux de rôle

Mais avant de commencer l'activité proprement dite, il faut d'abord l'introduire aux participants. L'étape de la préparation, dont nous avons traité plus haut, consistait pour l'animateur à préparer l'environnement et à se préparer lui-même ; il s'agit maintenant de préparer les joueurs.

\section{Lancement}

Introduire le jeu de rôle ou la simulation relève du grand art; c'est une étape qui se doit d'être très courte, mais qui risque d'être déterminante. Mettez de l'ambiance avec un coup d'envoi accrocheur sans toutefois verser dans le spectacle

Il y a toujours beaucoup d'informations à transmettre aux participants, mais il y a une limite à ce qu'ils sont capables d'assimiler. De plus, l'état d'excitation dans lequel ils se trouvent peut nuire à leur capacité de se concentrer sur ce que vous dites, et il n'est pas certain qu'ils retiennent l'essentiel de votre propos à moins qu'il ne soit très explicite.

Le premier conseil à suivre consiste donc à restreindre les explications au strict minimum. Certaines instructions particulières peuvent souvent être données en cours d'activité à mesure qu'elles s'appliquent; le piège à éviter est celui de vouloir tout dire dès le départ.

Commencez par décrire globalement ce qui est attendu des joueurs ; ceux-ci pourront par la suite interpréter les consignes que vous leur transmettrez en fonction du schéma intégrateur qu'ils se seront mentalement construit et l'information sera pour eux plus significative.

Une autre façon de s'assurer que l'information reçue est bien comprise consiste à démontrer à l'aide d'exemples les différents comportements à adopter. L'animateur peut demander 
Jeu, simulation et jeu de rôle

à un participant de venir le rejoindre et de l'assister dans la démonstration ; cela sécurise les autres et leur donne confiance dans leur capacité de performer dans ce mystérieux « monde parallèle » que vous leur proposez.

Les participants sont anxieux et leur esprit est en effervescence : comment cela va-t-il se passer ? Les apprenants savent généralement à quoi s'attendre d'un cours magistral, mais peu d'entre eux ont une idée claire de ce qui les attend lors d'une simulation, par exemple. En tant qu'animateur, vous devez vous montrer empathique à leur égard et exprimer votre compréhension de l'état d'incertitude et d'appréhension des joueurs.

Ainsi, il convient d'expliquer qu'une certaine confusion est probable au début de l'activité et que la situation évoluera rapidement jusqu'à devenir une seconde nature pour tous. Cela prédispose les individus à s'engager dans l'activité sans trop craindre de se tromper malgré le désordre initial caractéristique de ce genre d'événement.

Les participants apprécieront que vous leur annonciez la durée totale de l'activité ainsi que les balises de temps pour chaque étape, s'il y a lieu. Afin d'épargner au participant le stress supplémentaire d'avoir à gérer son temps, annoncez par la même occasion que c'est vous qui allez agir comme chronométreur (ou qu'un assistant le fera) et que vous donnerez des signaux appropriés aux moments clés.

Comme le matériel est la plupart du temps nouveau ou associé à une symbolique particulière pour les fins de l'activité, il est nécessaire de définir la signification des accessoires, symboles, etc., qui seront utilisés. Distribuer ce matériel au moment où l'on en explique l'usage, et pas avant, permet d'éviter toute distraction des joueurs pendant les autres explications. Idéalement, l'activité s'enclenchera immédiatement après. 
Préparation, animation et exploitation des simulations et des jeux de rôle

Il faut s'assurer que tous ont bien saisi l'ensemble des consignes avant de commencer. Il faut alors répondre à toute question ayant directement trait à la mécanique de l'activité et remettre à plus tard les éclaircissements sur la portée symbolique ou philosophique et autres considérations qui relèvent de l'interprétation. Quelques courtes questions très simples que vous poserez aux participants vous permettront d'évaluer leur maîtrise de cette mécanique :

- Combien vaut telle carte ou tel jeton?

- Quand a-t-on le droit de poser tel ou tel geste?

- Que doit-on faire dans tel ou tel cas?

Tout cela doit se faire dans un climat d'enthousiasme, où chacun est toutefois conscient de la valeur que revêt l'activité sur le plan pédagogique. À cet égard, pour certaines clientèles, il y a lieu que l'animateur explique brièvement le bien-fondé du jeu de rôle ou de la simulation en situation d'enseignement ou de formation; insistez surtout sur l'importance de l'apprentissage centré sur l'expérience de l'individu et sur l'avantage de la pédagogie par la découverte (l'approche inductive). Quant à la portée pédagogique de l'activité utilisée, elle peut être annoncée durant l'introduction ou n'être révélée qu'après coup afin de ne pas vendre la mèche lorsqu'il est préférable de garder le punch pour la fin.

\section{Déroulement de l'activité}

Le jeu de rôle et la simulation sollicitent la participation active des apprenants, mais cela ne signifie pas que l'enseignant soit pour autant réduit à un rôle plus passif, bien au contraire. L'animation de la période de jeu proprement dite requiert du doigté et du jugement. Bjur (1977) compare cette tâche à celle d'un jongleur : maintenir en état d'équilibre plusieurs facteurs 
Jeu, simulation et jeu de rôle

à la fois. Thiagarajan (1977) propose plusieurs dimensions avec lesquelles l'animateur doit « jongler » ; nous retenons particulièrement celles-ci :

- $\quad$ systématisation versus souplesse ;

- rapidité versus relaxation ;

- collaboration versus compétition;

- apprentissage versus agrément ;

- collectivité versus individualité ;

- omniprésence versus effacement.

Systématisation versus souplesse

L'animateur doit avoir un plan détaillé et systématique du déroulement de l'activité tout en étant prêt à y déroger ou tout simplement à adopter un scénario différent selon la dynamique qui opérera dans le feu de l'action. Prévoir les problèmes éventuels et être capable de s'adapter en pleine action est la marque des animateurs chevronnés. Après plusieurs utilisations du même jeu de rôle ou de la même simulation auprès de groupes différents, il peut être stimulant pour l'intervenant de sortir des sentiers battus et d'improviser des changements aux règles de l'activité sachant qu'en cas de pépin le retour au cadre initial est possible. L'application des règles avec lesquelles on choisit de fonctionner doit cependant être systématique et ne pas faire l'objet d'écart.

\section{Rapidité versus relaxation}

D'un côté, une activité au tempo trop lent est démotivante, voire ennuyeuse, de l'autre, un rythme trop rapide risque de compromettre les apprentissages visés. Dans les conditions idéales, l'animateur est à l'écoute du rythme naturel du groupe 
Préparation, animation et exploitation des simulations et des jeux de rôle

et enclenche les différentes étapes en temps opportun. Il est cependant rarement possible de faire cela à l'intérieur des limites de temps dont on dispose. L'animateur doit pouvoir accélérer le tempo sans être stressant ou menaçant et, inversement, il doit savoir ralentir les ardeurs des individus ou équipes survoltés sans miner leur intérêt pour le jeu de rôle ou la simulation. L'utilisation d'un chronomètre ou d'une horloge et le respect des balises de temps sont généralement des atouts précieux et évitent d'avoir à se précipiter à la fin de l'activité parce qu'on a perdu du temps au début.

Afin de composer avec les différences interindividuelles ou interéquipes concernant le temps requis pour réaliser une tâche, l'animateur pourra donner des exercices supplémentaires à ceux qui terminent plus vite ou leur demander de prêter main-forte aux autres lorsque l'activité le permet.

\section{Collaboration versus compétition}

Le phénomène de la compétition a fait l'objet de nombreuses critiques. Nous craignons qu'elle ne dégénère en animosité entre les joueurs ou que ceux-ci n'en viennent à perdre de vue l'objectif visé, mais nous savons aussi qu'elle crée une certaine émulation qui incite au dépassement personnel. Il y a donc lieu d'envisager la compétition comme une réalité de la vie qui, contrôlée, est un stimulant pouvant nourrir le jeu de rôle ou la simulation et pouvant les détruire également, lorsqu'on en perd le contrôle. Il faut, par conséquent, contrôler la compétition.

Il faut d'abord dédramatiser la défaite et, jusqu'à un certain point, déglorifier la victoire. Il est bon d'insister sur la valeur du processus pour l'apprentissage et de donner crédit aux individus ou équipes ayant fourni un effort particulier sans avoir pour autant gagné. Il est également possible de décerner des 
Jeu, simulation et jeu de rôle

mentions honorables en fonction de critères différents de celui qui mène à la victoire. Vous pouvez même établir des normes différentes pour différents individus ou inciter les joueurs à décider eux-mêmes du seuil de performance visé (dans le cas où l'activité leur est familière).

En outre, la collaboration entre les joueurs peut être grandement stimulée par le recours à une évaluation de performance non pas normative (telle équipe meilleure que telle autre) mais critériée (limite de temps, record à battre, etc.). Cela dit, les simulations et jeux de rôle posent beaucoup moins ce problème que les jeux tout courts.

\section{Apprentissage versus agrément}

Contrairement à la croyance populaire, les aspects pédagogiques et récréatifs ne sont pas incompatibles. Dans bien des cas, le premier s'exerce tout à fait inconsciemment chez le participant qui s'investit à fond dans le second. Il y a cependant lieu d'examiner consciencieusement le matériel qu'on utilise et d'identifier ses lacunes potentielles à l'égard du caractère pédagogique. Des exemples de telles lacunes sont un lien faible entre le système de pointage et les objectifs d'apprentissage ou encore une trop grande place tenue par la chance dans l'issue du jeu de rôle ou de la simulation.

Souvent, le moment d'apprentissage le plus riche est le debriefing (dont il sera question plus loin). Il arrive, par ailleurs, que des détails importants pour alimenter ce debriefing soient escamotés en cours d'action, ce qui nécessite une intervention immédiate du meneur qui doit être à l'affût de toute dérogation au scénario susceptible d'altérer la valeur pédagogique du jeu de rôle ou de la simulation. 
Préparation, animation et exploitation des simulations et des jeux de rôle

$\mathrm{Si}$, à l'inverse, le jeu de rôle ou la simulation s'enlise et revêt un caractère exagérément didactique, l'animateur peut ajouter $\mathrm{du}$ piquant par des points de boni ou autres astuces du genre ou par une petite mise en scène qui engage la participation dans un climat plus fantaisiste.

\section{Collectivité versus individualité}

Si l'animateur se doit d'être sensible à ce qui se dégage du groupe, il ne doit pas perdre de vue qu'au-delà du groupe il y a des individus. Dès l'étape de l'introduction, il faut savoir repérer les personnes qui n'ont pas saisi aussi rapidement que les autres la démarche à suivre et qui peuvent avoir besoin de plus d'assistance pour s'intégrer à l'action. Inversement, les personnes animées d'une grande curiosité et d'un certain esprit d'aventure peuvent menacer la bonne marche de l'activité (en obtenant prématurément des informations destinées à n'être connues que de l'enseignant, par exemple) ; on peut canaliser cet enthousiasme excédentaire en attribuant à ces apprenants des tâches spéciales (comme le chronométrage, le calcul des points, etc.) en plus de leurs tâches régulières.

$\mathrm{Si}$, parfois, il faut faire abstraction des différences individuelles par souci d'équité et de respect des personnes, il convient dans d'autres occasions de les reconnaître explicitement au moyen de handicaps où le joueur plus faible dispose de plus de ressources ou de plus de temps que le plus fort ; il va de soi que de telles modifications doivent recevoir l'assentiment de tous les joueurs concernés.

Le moment où les équipes se forment est stratégique pour une intervention subtile de l'animateur visant à équilibrer les chances de succès. Cela est particulièrement vrai dans le cas des jeux de rôle faisant intervenir plusieurs personnes ; 
Jeu, simulation et jeu de rôle

l'animateur peut attribuer les rôles de manière à restreindre la visibilité des personnes reconnues pour être très volubiles et favoriser celle des plus timides.

\section{Omniprésence versus effacement}

Le terme anglais game facilitator désigne bien la véritable fonction de l'animateur une fois l'activité lancée: celle de faciliter le déroulement et non de le diriger ou de le contrôler. Ainsi, l'animateur ne devrait se manifester et n'être visible que lorsque c'est absolument nécessaire, laissant la vedette aux participants. Ce faisant, il doit, toutefois, être d'une grande disponibilité pour les joueurs dès qu'ils ont besoin d'une aide quelconque. Il s'agit donc d'être discret tout en étant attentif et prêt à intervenir.

L'animateur qui connaît sa tendance à trop parler doit tenter de s'éclipser davantage et s'occuper à quelque chose d'utile comme noter les comportements qui se manifestent afin de pouvoir les relever lors du « debriefing ».

Il arrive que le jeu de rôle ou la simulation prennent un envol tel que vous passiez complètement inaperçu dans un brouhaha devenu incontrôlable, et que vous ayez, par ailleurs, une importante annonce à faire. Plutôt que de tenter d'enterrer le bruit en élevant le ton, éteignez simplement la lumière quelques secondes: l'effet est assez remarquable sur l'apaisement général et l'attention dont vous gratifieront les participants ! Cela dit, assurez-vous que tous sont attentifs à vos propos et que personne ne profite du temps mort pour procéder à quelque échange ou autre opération du genre. L'utilisation de transparents projetés sur écran (autant en profiter tandis que les lumières sont éteintes) est un excellent moyen de passer votre message qui devra être court (pour ne pas perdre l'élan acquis). 
Préparation, animation et exploitation des simulations et des jeux de rôle

Une fin brusque convient généralement. Les joueurs peuvent très bien garder le matériel en leur possession pendant quelque temps après la fin de l'activité; demandez-leur de vous le remettre seulement à la fin de la discussion et invitez-les plutôt à s'asseoir. Les quelques secondes pendant lesquelles les chaises sont disposées en cercle suffisent à faire descendre la vapeur et à retourner à un climat propice à l'amorce d'une discussion. C'est la prochaine étape, le « debriefing », qui constitue le premier volet de l'exploitation du jeu de rôle ou de la simulation.

\section{Exploitation}

Afin de tirer un profit maximal de l'activité qui vient de se terminer, il faut entamer sur-le-champ une discussion en plénière qu'on appelle «debriefing »; il faut battre le fer pendant qu'il est chaud et saisir l'occasion unique que fournit la présence de toutes ces personnes qui ne demandent qu'à s'exprimer et à partager leurs impressions.

Vous voudrez ensuite recueillir auprès des joueurs des commentaires sur l'activité elle-même en les invitant à en faire l'évaluation; les résultats de cet exercice vous seront utiles pour la tenue de la prochaine joute que vous animerez.

Enfin, des activités pédagogiques complémentaires se grefferont vraisemblablement au jeu de rôle ou à la simulation qui se termine. Voyons maintenant un à un ces trois volets de l'exploitation des jeux de rôle et simulations.

\section{Debriefing}

Le terme « debriefing » semble consacré par l'usage chez les concepteurs et les utilisateurs de jeux de rôle et de simulations. Il couvre une réalité plus large que son équivalent français 
« compte rendu ». Le terme « retour-synthèse » rejoint assez bien l'idée mais ne semble pas réussir à s'imposer dans le milieu.

Le debriefing est une discussion menée dans la foulée du jeu de rôle ou de la simulation comme tels et qui doit y être intimement associée afin de favoriser l'atteinte des objectifs d'apprentissage poursuivis.

Environ $30 \%$ de la durée totale de l'activité devrait être réservée au debriefing, ce qui est considérable: dans le cas d'une durée totale de deux heures et demie, cela représente 45 minutes. L'objectif du debriefing ne doit jamais être perdu de vue par l'animateur: fournir l'occasion aux participants de réfléchir sur l'expérience qu'ils viennent de vivre et d'en tirer des conclusions utiles pour leur compréhension de la réalité. Il est extrêmement important que l'animateur prenne toutes ses décisions, planifie toutes ses interventions en fonction de cet objectif. Dans la pratique, cela se traduit surtout en un rôle de facilitateur aussi discret et attentif que possible.

Durant le debriefing, même si la parole est aux joueurs, l'animateur doit orienter le cours des choses. Les étapes suivantes constituent une suite logique à laquelle il ne faut pas déroger si l'on veut atteindre ses objectifs:

- la catharsis (libération des émotions) ;

- la description (ce qui s'est passé, quand, comment) ;

- l'analyse (la relation entre l'activité et le monde réel) ;

- la généralisation (focus sur la réalité et les principes qui la caractérisent).

Le passage d'une étape à l'autre, dans la séquence, doit se faire le plus naturellement possible, mais c'est la responsabilité de l'animateur d'aider le groupe à bien cheminer. Il y a 
Préparation, animation et exploitation des simulations et des jeux de rôle

lieu de faire évoluer la discussion forçant le passage d'une étape à une autre lorsque le groupe a tendance à piétiner ou de ramener le débat aux considérations pertinentes lorsqu'on s'en écarte.

\section{Catharsis}

La catharsis, c'est la libération des tensions, la libération des émotions. Durant cette étape, les participants expriment librement leurs sentiments, réagissent avec leur émotivité. Personne ne doit être forcé à le faire, mais tous doivent sentir qu'ils en ont la possibilité.

L'animateur doit être vigilant et voir à restreindre les interventions inconvenantes des participants. Il faut, plus particulièrement, empêcher toute attaque personnelle entre les joueurs, de même que toute intellectualisation; l'analyse suivra sous peu et il s'agit à cette étape de s'exprimer et non d'expliquer.

Dans le cas où personne n'ose commencer, l'animateur peut briser la glace en livrant ses propres impressions; il peut ensuite s'enquérir auprès des joueurs si quelqu'un a ressenti la même chose ou le contraire. L'animateur peut également lancer quelques mots clés (frustration, découragement, insécurité, puissance, etc.) qui sont autant de perches qu'il tend aux participants qui sont invités à réagir.

La catharsis ne doit pas s'éterniser : trois à cinq minutes tout au plus. L'animateur profitera de tout ralentissement pour passer à la deuxième étape, la description. 
Jeu, simulation et jeu de rôle

\section{Description}

Vous enclencherez cette étape à l'aide d'une question se rap-portant à la mécanique de l'activité. Cette question peut être adressée à un sous-groupe en particulier ("Un Alphan peut-il nous expliquer comment cela se passe chez les Betans ? ») ou être très générale, permettant à n'importe qui d'y répondre («Quel genre de difficulté rencontre-t-on comme visiteur?»).

Invitez les joueurs à identifier clairement le rôle qu'ils tenaient avant d'y aller d'une intervention, surtout si vous avez affaire à un grand groupe. Il est probable que leur angle de vue influence leur lecture de la situation; cet aspect pourra être soulevé ultérieurement.

\section{Analyse}

Laissez les gens amorcer l'analyse des éléments descriptifs exposés et proposer un début d'interprétation des principes sous-jacents dès qu'ils le font spontanément. Certains auteurs dans le domaine du jeu et de la simulation ne font d'ailleurs aucune distinction entre ces deux étapes (Greenblat et Duke, 1975).

Afin de maximiser les retombées de cette étape importante, rédigez à l'avance une liste de principes que le modèle véhiculé par le jeu de rôle ou par la simulation illustre. En laissant les joueurs en relever d'eux-mêmes un certain nombre et en complétant par ceux qui auront été négligés, vous vous assurez de couvrir la matière tout en permettant à l'apprenant de contribuer à la discussion à partir de sa propre perception. D'ailleurs, la richesse d'une telle démarche tient à la possibilité que les participants établissent des liens différents des vôtres tout en étant aussi valables et pertinents. 
Préparation, animation et exploitation des simulations et des jeux de rôle

Durant cette étape, les références à la réalité et aux principes qui la régissent se multiplieront et bientôt vous observerez un va-et-vient continuel entre des situations tirées du jeu de rôle ou de la simulation et leur pendant dans le monde réel. Vous pouvez contribuer à systématiser ce parallèle tout en laissant le soin aux participants de proposer leur interprétation personnelle en alimentant le débat de questions courtes et précises.

Afin d'augmenter l'étendue des leçons à tirer de la situation, complétez le tableau par une série de questions à partir d'hypothèses ; en voici des exemples :

- Que serait-il arrivé si telle ou telle chose s'était produite?

- En l'absence de telle ou telle contrainte sur quelle autre issue aurait-on pu déboucher?

- Et si...? (Invitez les joueurs à formuler eux-mêmes de telles hypothèses en complétant l'énoncé.)

Les analogies avec une variété de situations sont bienvenues à ce stade-ci. Il arrive que la réalité à l'étude soit assez étrangère à l'expérience de vie des participants. Les principes régissant un phénomène s'appliquent cependant souvent à des situations plus familières pour l'individu ; bien qu'on dise de toute comparaison qu'elle est odieuse, cela ne l'empêche pas d'être, par ailleurs, fort instructive pourvu qu'on sache faire preuve de discernement.

\section{Généralisation}

Une fois le parallèle clairement établi entre le monde réel et le modèle qu'en constitue le jeu de rôle ou la simulation, l'animateur a une excellente occasion d'élaborer sur la réalité et de transmettre un supplément d'information sur le sujet aux apprenants, ceux-ci étant tout disposés à le recevoir et à y 
contribuer eux-mêmes. Leur curiosité a été éveillée, ils se sont de plus réchauffés en participant à l'analyse de la situation de jeu; leur implication dans la poursuite de l'investigation de l'objet d'étude en est donc grandement favorisée.

Il faut toutefois se garder d'allonger la sauce et d'entreprendre une étude exhaustive du phénomène autour duquel s'articulait l'activité. Le jeu de rôle ou la simulation doit demeurer l'élément prégnant de la rencontre, et il y a fort à parier que les participants se remémoreront l'expérience avec plaisir.

En marge du debriefing, l'animateur sera appelé à fournir une certaine rétroaction au groupe ou encore aux participants qui le solliciteront à l'occasion («Ai-je bien fait de faire ceci ou cela à tel moment ? »). La rétroaction vise avant tout à aider une personne à prendre conscience de sa manière de se comporter et des façons de l'améliorer. Il est bon de retenir les points suivants lorsqu'on est appelé à donner un feedback :

- être spécifique plutôt que général (employer des exemples concrets et rapporter les paroles du joueur quand on le peut) ;

- décrire plutôt qu'interpréter (se limiter au comment et éviter le pourquoi) ;

- faire référence aux comportements susceptibles d'être modifiés sans demander de les modifier (les individus peuvent décider euxmêmes si la situation commande un changement de comportement) ;

- avoir toujours comme intention d'aider et non d'offenser ou de sanctionner l'individu.

Le debriefing est une étape généralement très riche en retombées pédagogiques et ce que les participants en retireront déborde souvent les apprentissages initialement visés. Il est donc très rentable de bien le préparer pour en tirer le meilleur parti 
Préparation, animation et exploitation des simulations et des jeux de rôle

possible. La liste de vérification qui se trouve à la fin de ce chapitre fournit plusieurs points à retenir pour la tenue du debriefing.

\section{Évaluation formative du jeu de rôle ou de la simulation}

L'enseignant ou le formateur désire toujours proposer des activités qui conviennent aux apprenants; leur demander de prendre part à l'évaluation formative des jeux de rôle et simulations auxquels ils viennent de participer est sans contredit le meilleur moyen pour le faire.

Cette évaluation peut être faite de vive voix et de façon très informelle ou encore faire l'objet d'un questionnaire très systématique. Le meilleur moyen sera celui qui permettra à l'enseignant de recueillir les informations qu'il juge importantes.

Bien que chaque jeu de rôle ou simulation commandera des questions particulières, les questions qui suivent peuvent s'appliquer dans la majorité des situations:

- Qu'avez-vous aimé le plus dans cette activité ?

- Qu'avez-vous aimé le moins ?

- Quel changement suggérez-vous d'apporter à l'activité pour la rendre plus intéressante?

- Quel changement suggérez-vous d'apporter à ce jeu de rôle (ou à cette simulation) pour le rendre plus efficace dans l'atteinte des objectifs poursuivis?

- Quels sont les autres objectifs que ce jeu de rôle permet d'atteindre?

De plus, vous pouvez demander aux apprenants de coter l'activité sur plusieurs aspects : pertinence, valeur pédagogique, 
plaisir qu'elle procure, validité (dans le cas de la simulation), rapport chance-science, etc. Dans le cas de questionnaires écrits, il est avantageux de remplacer les cotes numériques par des continuums où vous demandez à l'apprenant de situer l'activité entre deux pôles. Par exemple :

Quel lien existe-t-il entre l'application d'une stratégie et les résultats concrets lors de l'activité ?

Aucun lien _. . . . . . . . . . . . Très fort lien (causalité directe)

La simulation comporte-t-elle des éléments d'une complexité qui ne se retrouve pas dans la réalité qu'on a modélisée?

Pas du tout . . . . . . . . . . . Beaucoup

\section{Suivi}

Nous avons parlé dans ce chapitre et dans les chapitres précédents du pouvoir de motivation du jeu de rôle et de la simulation. On doit profiter de cette motivation et poursuivre la séquence d'enseignement sur l'élan fourni par l'activité.

On fera donc sporadiquement référence à l'expérience vécue au cours de l'activité afin de rendre plus significatives les activités d'enseignement subséquentes. Partant du fait que les joueurs se rappelleront le jeu de rôle ou la simulation facilement et que ce souvenir leur sera agréable, toute activité que vous lui associerez bénéficiera d'un préjugé favorable et aura de meilleures chances de succès.

Après avoir utilisé ce type d'intervention pédagogique à quelques reprises, il sera parfois possible de faire participer les apprenants à la préparation ou même à la conception de jeux de rôle ou de simulations ; un tel travail permet une étude approfondie de certains sujets dans un contexte très stimulant pour l'apprenant. 
Préparation, animation et exploitation des simulations et des jeux de rôle

Comme avec toute autre formule pédagogique, le jeu de rôle et la simulation perdent de leur efficacité si l'on en abuse ; il est donc important de ménager une place pour d'autres activités pédagogiques de nature complémentaire afin de multiplier les chances de succès dans la poursuite des objectifs pédagogiques.

En conclusion, afin de tirer le maximum d'une simulation ou d'un jeu de rôle, il importe de réussir l'activité elle-même et le debriefing qui la suit.

Rappelons également que le meilleur gage de succès en ce qui a trait au jeu proprement dit, c'est une préparation appropriée. Cette préparation peut se résumer ainsi :

- se préparer soi-même (bonne maîtrise du jeu de rôle ou de la simulation par l'animateur) ;

- préparer l'environnement (lieu, temps, matériel);

- préparer les participants (bonne introduction).

La suite des événements dépend de l'habileté de l'animateur à maintenir un juste équilibre sur divers tableaux. Il faut qu'il soit bien préparé et systématique, tout en étant capable de faire preuve de flexibilité en temps opportun. Le rythme suivant lequel se déroule l'activité doit être assez rapide pour arriver à quelque chose, mais une atmosphère de détente doit tout de même régner. La compétition doit être canalisée. On doit doser les aspects pédagogiques et récréatifs. L'animateur se doit d'être attentif autant au groupe dans son ensemble qu'à chacun des individus qui le composent. Et afin de réussir tout cela, l'animateur doit s'effacer pour faire place aux participants, mais réapparaître dès que son intervention est nécessaire. 
Mais son travail ne s'arrête pas là ! Le debriefing qui suit est un moment privilégié d'apprendre et l'animateur en oriente le déroulement tout en complétant les interventions de manière à couvrir l'ensemble de la matière.

L'évaluation formative du jeu de rôle ou de la simulation complète le cycle et constitue la première contribution à la planification de la prochaine activité du même genre. Quant à l'étude du contenu d'apprentissage, elle se poursuit en profitant du momentum par diverses activités de suivi.

Au tableau 4, nous présentons une liste de vérification pour guider votre démarche lorsque vous utilisez un jeu de rôle ou une simulation. Nous vous suggérons d'en faire une photocopie à chaque fois que vous aurez à animer une activité de ce genre. Cette liste, que nous avons voulue exhaustive, vous étonnera peut-être avec tous les points qu'elle contient. Il est probable que certains de ces éléments ne s'appliquent pas à votre situation; ne vous y attardez pas. Ils s'appliqueront peut-être dans le cadre d'un autre jeu de rôle ou d'une autre simulation. 
Préparation, animation et exploitation des simulations et des jeux de rôle

Voici enfin quelques questions que doit se poser l'animateur durant le debriefing :

- Tous les participants ont-ils la chance d'être entendus ou est-ce qu'une ou deux personnes seulement monopolisent la discussion?

- La discussion est-elle pertinente à l'expérience vécue et aux réactions des participants?

- La discussion évolue-t-elle à un bon rythme ou y a-t-il enlisement sur un sujet?

- Le groupe s'en va-t-il quelque part?

Voilà qui termine notre propos sur la préparation, l'animation et l'exploitation pédagogique des jeux de rôle et des simulations. Bien qu'ils requièrent habituellement davantage de temps que les jeux ou que les autres formules pédagogiques, nous pouvons dire qu'il s'agit généralement d'un investissement rentable.

Dans le chapitre qui suit, nous cédons la parole à des enseignants qui donnent leur appréciation de la rentabilité et de l'efficacité de ces stratégies d'enseignement. 
Jeu, simulation et jeu de rôle

Tableau 4 : Liste de vérification pour la préparation, l'animation et l'exploitation pédagogique d'un jeu de rôle ou d'une simulation

\section{La préparation (avant l'activité)}

A-La planification (plusieurs jours avant)

- Situer l'activité dans une séquence d'enseignement.

- Prévoir les liens avec les autres activités de la séquence.

- Étudier soigneusement la mécanique du jeu de rôle ou de la simulation.

- Modifier le jeu, au besoin, en fonction des besoins particuliers.

- Organiser les ressources humaines et matérielles :

- contacter les gens qui collaboreront;

- attribuer un rôle ou une fonction à chacun;

- réserver les locaux;

- commander le matériel non disponible.

B - Les préparatifs (quelques heures ou quelques minutes avant le début de l'activité)

- Confirmer les aspects logistiques.

- Aménager les lieux.

- Préparer et installer le matériel :

- rassembler les accessoires, outils, papiers, etc.;

- inventorier le matériel et ne plus y toucher.

- Constituer un horaire précis.

- Dresser un aide-mémoire à utiliser en cours d'activité.

- Intérioriser les étapes du déroulement de l'activité.

- Pratiquer les manipulations et les automatiser.

- Rédiger, au besoin, un court texte d'introduction.

\section{L'animation (pendant l'activité)}

A - L'introduction de l'activité

- Créer une ambiance.

- Être bref.

- Aller du général au particulier.

- Annoncer la durée approximative de l'activité.

- Vérifier la compréhension des participants. 
Préparation, animation et exploitation des simulations et des jeux de rôle

B - Le déroulement de l'activité

- Intervenir le moins possible.

- Prendre note de ce qui se produit, des moments clés et des remarques particulièrement révélatrices émises par les participants.

\section{L'exploitation (après l'activité)}

\section{A - Le debriefing}

- La catharsis (les participants expriment leurs sentiments et émotions)

- Procéder tout de suite après le jeu de rôle ou la simulation.

- Ne forcer personne à s'exprimer.

- Permettre à tous de le faire.

- Briser la glace, au besoin, avec ses propres commentaires.

- Arrêter toute attaque personnelle.

- Arrêter toute intellectualisation.

- Ne passer que 5 à 7 minutes environ sur cette étape.

- La description (qui porte sur les aspects mécaniques)

- Amorcer cette étape à l'aide de questions adressées au groupe.

- Demander à ceux qui interviennent d'identifier d'abord le rôle qu'ils tenaient.

- Demander qu'on fournisse des exemples concrets.

- L'analyse et l'interprétation (liens avec la réalité)

- Préparer d'avance une liste de principes à aborder.

- Laisser aux participants le loisir de proposer leur propre interprétation.

- Compléter en fonction des points négligés.

- Étendre l'analyse au moyen d'hypothèses ("Et si...?").

- S'adresser au vécu des participants au moyen d'analogies avec des phénomènes qui leur sont familiers.

- La généralisation (étude plus poussée de la réalité)

- Élaborer sur la réalité au-delà des aspects couverts par le jeu de rôle ou la simulation.

- Généraliser à des situations plus larges ou analogues.

- Éviter de traîner en longueur sur ce point.

B - L'évaluation

C - Le suivi 



\section{Chapitre 8}

\section{Témoignages d'enseignants}

\section{TÉMOIGNAGE D'UNE ENSEIGNANTE DU PRIMAIRE}

Je m'appelle Micheline Rancourt, j'enseigne au primaire depuis plus de trente ans. Tout au long de ces années, j'ai fait différentes constatations quant à la place du jeu dans mon enseignement. Je veux partager avec vous le fruit de mes réflexions.

Les enfants du primaire ont de 6 à 12 ans. Ils aiment jouer. Pour eux toute situation, tout objet ou tout événement peut susciter une idée de jeu. L'enfant joue sans arrêt. L'enseignante du primaire doit elle aussi aimer jouer. Elle doit avoir du plaisir à jouer avec les mots, les images et les expressions. Ainsi elle ne pourra que rejoindre la passion des enfants. Elle sait qu'elle doit faire jouer le jeune. Même si ce n'était que pour les faire se reposer, la titulaire fera jouer les enfants. Elle n'utilisera, pas toujours le jeu de la même façon mais il sera présent. À la fin de la journée, on entendra quelquefois des écoliers s'écrier : «Aujourd'hui, on n'a fait que s'amuser ! »

Vers la fin des années 1970, les jeux avaient une très grande place au primaire. L'enseignement par ateliers était très à la mode et les jeux didactiques étaient une bonne façon de faire 
travailler les enfants individuellement sur la consolidation de certains acquis. La plupart des jeux étaient conçus et réalisés par les enseignants. Ce travail était exigeant. Très souvent les enseignantes passaient des heures à composer, dessiner, colorier, plastifier des jeux attrayants. Les écoliers réalisaient ces activités en quelques minutes et lorsque l'on voulait vérifier les connaissances visées, on constatait souvent que celles-ci n'étaient pas véritablement acquises. Nous étions encore dans le vent de l'école active. Nous étions convaincus que les enfants pouvaient apprendre seuls. Il suffisait qu'on les place dans une situation stimulante et le jeu répondait à cet objectif. De récentes études nous ont démontré qu'il ne suffisait pas de stimuler l'enfant pour qu'il apprenne, il fallait le rendre conscient de ses apprentissages et lui apporter le soutien nécessaire tout au long de ce processus. Nous avons dû nous réajuster et réviser notre manière d'intervenir.

Depuis maintenant près de quinze ans, nous avons appris à travailler avec les démarches des différents programmes. Nous avons été sensibilisés et très souvent habilités à l'enseignement stratégique et à l'enseignement coopératif. Tous ces nouveaux courants nous ont amenés à penser différemment et graduellement à changer notre manière d'enseigner. Malgré tous ces changements, le jeu reste présent. Nous ne pouvons l'oublier car l'enfant lui-même nous y conduit.

Le jeu se retrouve régulièrement dans une démarche d'apprentissage. Nous le retrouvons à toutes les étapes de ces démarches mais plus particulièrement dans les mises en situation et dans les activités d'exercisation. Je vais illustrer cette observation par quelques exemples.

Une stagiaire veut enseigner la notion de frontière et région. Elle se déguise et devient le cowboy Gerry. Ce cowboy a un grave problème. Ses animaux ne savent plus où aller. Un 
chien a eu un coup de sabot de cheval. Les poules volent la nourriture des lapins et les cochons ont sali les beaux draps blancs que sa femme venait tout juste de laver. La stagiaire remet aux enfants un plan de son ranch. Elle y a situé la maison, la grange et la rue. Elle leur remet aussi une enveloppe dans laquelle elle a mis des petites vignettes d'animaux. Les enfants doivent lui proposer une solution afin que tous les animaux vivent en paix.

En français, les enfants ont composé durant un exercice de consolidation des phrases avec le pronom « Je » Nous partons de ces phrases pour faire un jeu de mime. Un enfant pige une phrase et mime le geste qui y est décrit. Les autres écoliers essaient de deviner. L'enfant qui a deviné vient écrire ce geste au tableau en employant le pronom « $\mathrm{Tu}$ ». Ainsi nous avons démarré la conjugaison des verbes avec le pronom « $\mathrm{Tu} »$.

Ces deux activités démontrent l'utilisation du jeu comme mise en situation à un nouvel apprentissage. La plupart du temps, ces jeux permettent aux écoliers de s'implanter et de s'engager rapidement dans l'apprentissage d'une nouvelle connaissance ou d'une nouvelle habileté. Si le jeu a créé un certain déséquilibre chez l'apprenant, il sera curieux de voir ce qui vient. Il sera prêt pour apprendre.

$\mathrm{Au}$ primaire, le jeu est utilisé très régulièrement dans la consolidation de nouveaux acquis. À ce moment-là, le jeu permet à l'enfant d'utiliser les connaissances qu'il vient de découvrir. Tous les dérivés du bingo sont des exemples frappants. Le bingo des prénoms en première année permet au tout-petit de lire régulièrement les prénoms de ses amis et de les identifier rapidement. C'est la même chose pour le bingo des multiples. Ce jeu donne l'occasion aux écoliers de calculer vite et bien. 
Rappelons-nous les combats que nous faisions à la petite école. Tous alignés, nous attendions avec impatience et inquiétude le moment où la question nous serait posée. Nous encouragions nos coéquipiers, nous voulions gagner. C'est certain qu'il y a dans ce jeu comme dans d'autres une certaine compétition. Celle-ci n'est certainement pas néfaste si elle est utilisée avec discernement.

Il existe beaucoup de jeux sur le marché qui permettent à l'enfant d'exercer ses connaissances. Je pense au « Scrabble » à « Uno » ou au jeu de cartes conventionnel tout simplement. Ces jeux sont régulièrement utilisés en classe et ils permettent aux écoliers de s'amuser mais aussi de continuer à apprendre.

Ces jeux, que ce soit pour démarrer un apprentissage ou pour le consolider, sont utilisés de façon ponctuelle. Il y a aussi dans la vie au primaire une grande utilisation du ludique. Nous travaillons souvent dans l'imaginaire. Enfant comme adulte mettent à profit leur imagination et leur intuition.

Ce côté ludique est plus constant. Toutes les petites histoires que les enseignantes racontent pour que les enfants saisissent mieux un concept sont une belle illustration de l'utilisation du ludique. Souvenez-vous de l'histoire du petit « $\mathrm{n} »$ qui a peur $\mathrm{du}\langle\mathrm{b} »$ et du « $\mathrm{p}$ » alors il demande à son ami le « $\mathrm{m} »$ de le remplacer ou à toutes les images qui sont associées à chacun des sons.

Lorsque nous permettons aux enfants d'inventer un personnage ou un objet, nous les amenons dans un monde imaginaire, un monde très connu d'eux. Pensons à la fascination que la marionnette exerce sur l'enfant. Quand c'est celui-ci qui fabrique sa marionnette, qui l'habille, qui lui donne sa voix, c'est merveilleux. Il se crée entre l'enfant et sa création une magie. 
Cette magie donne toute une motivation à ce jeune apprenant. Il sera stimulé à composer un petit scénario pour faire vivre sa marionnette. Ce petit personnage pourra être le moteur pour plusieurs activités d'apprentissage.

Nous pouvons aussi faire jouer l'enfant en art dramatique. Nous pouvons alors faire des jeux tout simples comme le pantin, le miroir ou l'improvisation. À ce moment-là, les enfants s'amusent et peuvent se permettre de vivre des rêves et des angoisses. Ces jeux sont bénéfiques et amusent beaucoup les jeunes. Il y a aussi les pièces de théâtre que l'enfant apprend. Il doit tout d'abord mémoriser son texte puis travailler avec d'autres acteurs pour faire une réussite collective. À ce moment-là, ce petit comédien s'amuse, joue à être quelqu'un d'autre. Dans cet exercice, l'écolier utilise ses connaissances afin de bien comprendre la pièce qu'il joue mais il s'exerce aussi à une vie sociale où chacun a un rôle important et différent à jouer.

Le jeu est présent et vivant au primaire. Aucune enseignante ne peut dire le contraire. Car ces jeunes savent jouer Ils peuvent même nous enseigner

Comment vivre du merveilleux Rire, chanter, créer un monde tout bleu.

Micheline Rancourt enseignante à la Commission scolaire La Jeune Lorette 


\section{TÉMOIGNAGE D'UNE ENSEIGNANTE DU SECONDAIRE}

Enseignant l'anglais au secondaire, j'ai réalisé depuis longtemps qu'il est important que les activités d'apprentissage que je propose aux élèves soient variées, centrées sur eux, basées sur des expériences concrètes, près de leur vécu. Voilà pourquoi le jeu, la simulation, le jeu de rôle font partie régulièrement de mes stratégies d'enseignement.

La communication pédagogique doit être authentique autant que possible. Nous devons donc utiliser du matériel authentique et placer l'apprenant dans une situation qui, elle aussi, doit être authentique. Ce contexte est nécessaire pour faciliter l'interaction si importante dans le processus d'acquisition de connaissances et cela pas seulement en langue seconde.

Les activités d'apprentissage doivent idéalement faire appel à des habiletés de raisonnement et de créativité de la part de l'apprenant. On doit donc présenter une grande variété de tâches qui conduisent souvent à plusieurs solutions possibles. Il faut utiliser l'environnement de la classe et exploiter pleinement l'interaction sociale qu'il permet.

Ainsi, l'expérience à laquelle l'élève est invité suscite sa motivation, condition essentielle et préalable à tout apprentissage. Il faut arriver à créer une atmosphère non menaçante dans la classe et ainsi créer une situation propice à une plus grande réceptivité des apprenants. Un tel type d'environnement favorise chez l'élève le développement d'une image positive de soi et d'un véritable sentiment de compétence.

Personnellement j'utilise plusieurs techniques d'enseignement. Il n'y a pas de méthode idéale, chaque approche présente ses avantages et ses inconvénients par rapport aux objectifs visés. 
Avec mes élèves j'exploite tant l'exposé magistral, l'enseignement par les pairs, les projets que les mises en situation où les tournois, les jeux de rôle et autres jeux éducatifs occupent une place importante.

L'utilisation du jeu de rôle, par exemple, m'a toujours confirmé le potentiel de cette approche pédagogique. J'ai été à même de constater qu'il favorise non seulement l'acquisition de la maîtrise d'une langue seconde, mais aussi le développement de stratégies de résolution de problèmes ou d'habiletés en relations interpersonnelles. Il permet un apprentissage continu chez les participants et constitue un instrument privilégié pour agir sur les attitudes.

Une présentation adéquate des objectifs du jeu suscite en général l'intérêt et motive l'élève à s'engager activement dans l'exercice. La représentation de la situation problématique stimule l'individu et l'échange continuel entre les élèves multiplie les occasions d'apprendre.

Cette technique offre la possibilité d'expérimenter des stratégies, d'exploiter son répertoire de connaissances sur un sujet, de pratiquer une habileté, d'acquérir par l'imitation de ce que font les autres. La période de discussion qui suit permet d'ajuster les perceptions, de corriger les erreurs, de revenir sur le contenu d'apprentissage visé par l'activité et ainsi de le renforcer, d'en permettre un meilleur ancrage.

L'utilisation de jeux crée généralement un climat d'enthousiasme au sein du groupe, autant chez les élèves entre eux que dans la relation entre les élèves et l'enseignant. Ce haut niveau de motivation favorise le maintien de l'attention des participants sur les objectifs poursuivis. Par l'assignation de différents rôles pendant le jeu, les besoins différents de chaque individu peuvent aussi être pris en compte. 
Jeu, simulation et jeu de rôle

Le but ultime de toute démarche éducative est de faire en sorte que l'élève se découvre, qu'il acquière de la confiance, qu'il devienne autonome. Celui-ci se responsabilise lorsque, comme le permettent les différentes formes de jeux éducatifs, on le met en situation de se prendre en charge, de s'impliquer activement dans son apprentissage. Il est donc important de faire vivre des expériences pertinentes, d'utiliser des mises en situation qui conduisent à la transformation des éléments notionnels des programmes en connaissances bien intégrées par l'individu.

Elizabeth Vatcher enseignante à la Commission scolaire Des Chutes de la Chaudière 


\section{TÉMOIGNAGE D'UN ENSEIGNANT DU COLLÉGIAL}

\section{Dix années de simulation : observations et leçons}

Responsable de l'enseignement de l'histoire au cégep de Drummondville, j'ai pu pendant plus de dix ans utiliser des jeux de simulation dans plusieurs cours. En particulier, le cours d'histoire des relations internationales a fonctionné pendant de nombreuses sessions entièrement avec un jeu de simulation, sans cours magistral ou autre forme de méthode présentative. Cette expérience est certainement très rare, quoique pas complètement unique, car au moins un collègue américain a osé entreprendre la même démarche. En plus de cette expérience pratique, j'ai conduit, avec mon collègue et ami Dany Laveault, de l'Université d'Ottawa, trois expériences contrôlées sur l'impact et l'efficacité des jeux de simulation comme méthode d'apprentissage. Les lecteurs intéressés pourront retrouver les résultats parmi les lectures suggérées. Entre les contacts constants avec les étudiants et les résultats de ces recherches, je crois possible d'offrir trois observations ou leçons sur l'apprentissage, sur l'usage des simulations et sur les conditions qui rendent possible l'innovation.

L'apprentissage est une activité. Cette leçon fondamentale je l'ai apprise des cohortes successives d'étudiants qui se sont précipités avec énergie et enthousiasme dans le jeu de relations internationales. L'apprentissage est le produit de l'effort de celui qui apprend, et l'apprentissage est efficace quand l'esprit ludique est engagé. La langue française fait un paradoxe de l'évidence de mes années avec les jeux de simulation, que l'on ne travaille jamais aussi fort qu'en jouant. Cette expérience apporte aussi un soutien expérimental ou semi-expérimental à la notion que la forme et le contenu ne peuvent pas être séparés. 
C'est une notion à laquelle il faut souscrire pour utiliser les jeux de simulation. En effet, les simulations sont probablement sous-utilisées parce que l'on ignore trois conditions nécessaires à leur usage comme moyen d'apprentissage. Premièrement, il faut laisser aux simulations le temps d'agir, comme on fait pour d'autres méthodes : qu'arriverait-il si on inversait le temps accordé à une simulation dans un trimestre avec le temps accordé au cours « magistral » en donnant par exemple trois périodes de «magistral » et quarante de simulation? Deuxièmement, il faut adapter la méthode d'évaluation à la méthode d'apprentissage; un test à choix multiple est-il une bonne mesure de la résolution de problème caractéristique du jeu ? Troisièmement, il ne faut pas oublier que la maîtrise d'un nouvel instrument requiert du temps.

Par suite des recherches mentionnées ci-dessus, nous avons conçu l'idée que l'apprentissage se fait en plusieurs étapes. La première est l'apprentissage de la « grammaire » de l'outil, comme les règlements et le matériel du jeu. Tout comme les étudiants, dans un cours " magistral », doivent maîtriser les règles de la prise de notes, ils doivent prendre le temps de maîtriser les règles du jeu. Pendant la deuxième étape, les étudiants apprennent à coordonner ces règles et ce matériel en schémas stratégiques. La troisième étape est celle de la vérification expérimentale de ces schémas. À la quatrième étape, les étudiants commencent à créer des relations explicatives ou causales entre les stratégies employées et les résultats obtenus ; ils testent des hypothèses, même si souvent ils ne connaissent pas le mot. C'est à partir de cette étape que les étudiants utilisent le jeu pour apprendre. Une cinquième étape n'est pas atteinte par tous ; il s'agit de découvrir que la simulation, et son modèle, représente un choix de variables et de questionnement possible. Ces étapes, on l'aura constaté, doivent beaucoup à la psychologie développementale de Piaget. 
J'écris ce texte au passé, car il ne m'est plus possible d'utiliser les jeux de simulation. L'innovation, du moins dans une institution scolaire, n'est possible que dans une ambiance ouverte et prospère. Quand les ressources manquent et l'horizon s'assombrit, les humains reviennent aux bonnes vieilles méthodes ; il faut dire que l'évolution leur a peut-être donné raison jusqu'ici ! Mais pour ceux que le contexte et les ressources favorisent, je propose trois règles pour guider les efforts. Premièrement, tout usage des jeux de simulation devrait être accompagné de recherche organisée. L'éducation n'est pas une science exacte et il faut des faits et des données documentées. Ce genre de recherche doit être de la recherche sur le terrain, ce que Bronfenbrenner (1976) appelait jadis de la recherche écologique Deuxièmement, il faut prendre des risques. On pourrait soutenir qu'apprendre suppose par définition des tentatives et des erreurs, mais, de plus, on ne progresse jamais avec des demi-mesures. Ma décision de construire un cours entièrement avec un jeu de simulation était en bonne partie un saut dans le vide - mais je ne l'ai jamais regretté. Troisièmement, il faut faire un effort épistémologique. En éducation, comme en science en général, on ne peut pas changer seulement un aspect de la question. En changeant la méthode d'apprentissage, on est obligé de repenser le concept de contenu, le moyen d'évaluation, le rôle de l'enseignant, finalement, tout. Mais les découvertes, grandes et petites, sont par définition des chocs, des expériences limitrophes.

De mon expérience, j'ai tiré une dernière leçon, peut-être personnelle. L'innovation est presque toujours douloureuse, en tout cas pour l'innovateur, mais la transformation qu'il vit en vaut toujours la peine.

Pierre Corbeil, Ph.D. enseignant au cégep de Drummonduille 
Jeu, simulation et jeu de rôle

\section{Lectures suggérées}

BRONFENBRENNER, U. (1976). " The experimental ecology of education ». Educational Researcher, 9, p. 5-15.

CORBEIL, P. (1988). History and technology : new tools for new questions ». Simulation/Games for Learning, 18 (2), p. 127-134.

CORBEIL, P. (1995). Entreprendre par le jeu. Montréal : Les éditions Transcontinentales et la Fondation de l'Entrepreneurship.

CORBEIL, P. et D. LAVEAULT (1994). « Descriptive research, innovation and epistemology : 10 years of lessons learned on the use of simulation games ». International Play Journal, 2, p. $122-134$.

CORBEIL, P., D. LAVEAULT et M. ST-GERMAIN (1989). Jeux et activités de simulation. Ottawa : Agence canadienne de développement internationale.

LAVEAUlT, D. et P. CORBEIL (1986). " Psychopédagogie du jeu de simulation pour l'apprentissage de l'histoire ». Revue des sciences de l'éducation, 12 (1), p. 25-43.

LAVEAULT, D. et P. CORBEIL (1988). « Évaluation formative et innovation pédagogique : une réponse au défi de l'efficacité ». Association internationale de pédagogie universitaire, Montréal.

LAVEAULT, D. et P. CORBEIL (1990). « Assessing the impact of simulation games on learning : a step-by-step approach ». Simulation/Games for Learning, 20 (1), p. 42-54. 


\section{Conclusion}

Dans cet ouvrage, nous avons voulu définir et distinguer le jeu, la simulation et le jeu de rôle, cerner les caractéristiques particulières à chacune de ces formules pédagogiques et faire ressortir leurs avantages de même que leurs limites. Nous nous sommes attardés à des considérations importantes reliées à leur conception et nous avons décrit les conditions à mettre en place pour leur utilisation efficace et leur exploitation en situation d'enseignement.

Le survol des origines du jeu et de la simulation nous a permis de constater qu'il s'agit de modes de communication foncièrement naturels pour l'homme qui s'en est toujours servi spontanément pour représenter les différents éléments du monde qui l'entoure ; le jeu d'échecs, véritable modèle de la société médiévale, en est un exemple frappant.

Notre étude des fondements sur lesquels s'appuie leur utilisation en enseignement ou en formation a démontré que malgré les hauts et les bas que ces formules pédagogiques ont connus au fil des époques, de solides raisons justifient le recours au jeu et à la simulation comme instruments de transmission du savoir et du savoir-faire. Ils s'inscrivent naturellement dans le courant de pensée qui veut qu'apprendre à apprendre soit plus important 
que l'accumulation de connaissances dans un monde caractérisé par une accélération du changement et une explosion des connaissances.

Dès que l'on considère l'intellect non pas comme un contenant à remplir mais comme un outil à mettre au point, on ouvre la voie à la contribution des jeux et simulations. Ils conviennent particulièrement bien à une compréhension de la réalité dans sa globalité, qui va audelà d'une compréhension des éléments qui la composent; en ce sens, les jeux et simulations relèvent d'une approche systémique. Nous avons également vu comment la participation active de l'apprenant dans le processus éducatif favorise l'apprentissage, notamment celui par la découverte.

Nous espérons avoir suscité un intérêt pour le jeu, la simulation et le jeu de rôle chez les enseignants qui adhèrent à l'idée qu'une plus grande appropriation par les apprenants du processus d'apprentissage leur est bénéfique. Nous souhaitons beaucoup de succès et de plaisir à tous les concepteurs et utilisateurs de jeux et simulations en enseignement. 


\section{Bibliographie}

ALLEN, D. et K. RYAN (1972). Le micro-enseignement : une méthode rationnelle de formation des enseignants. Paris : Dunod.

ARTAUD, A. (1987). « La recréation du savoir ». Pédagogiques, vol. 7, nº 1, p. 9-22.

BANDURA, A. (1969). Principles of Behavior Modification. New York : Holt, Rinehard and Winston.

BECKER, H.A. et H.M. GOUDDAPPEL (1972). Developments in Simulation and Gaming. Wolvega (Pays-Bas) : Boom.

BELL, R.C. (1983). The Boardgame Book. London : Cavendish House.

BJUR, W. (1977). « So you want to try a simulation game ? Here are 'getting underway' suggestions ». Simulation/Gaming, mars-avril, p. 4-6.

BOWEN, D.D. (1987). " Developing a Personal Theory of Experiential Learning », Simulation \& Games, vol. 18, $\mathrm{n}^{\mathrm{0}} 2$, juin, p. 192-206.

BRIEN, R. (1994). Science cognitive et formation. $2^{\mathrm{e}}$ éd. SainteFoy : Presses de l'Université du Québec.

CHAMBERLAND, G. (1989). Étude des relations entre la pratique de jeux, les résultats scolaires et le statut socioéconomique d'étudiants de secondaire II. Mémoire de 
maîtrise en technologie de l'enseignement, Faculté des sciences de l'éducation de l'Université Laval.

CHAMBERLAND, G., LAVOIE, L. et D. MARQUIS (1995). 20 formules pédagogiques. Sainte-Foy : Presses de l'Université du Québec.

CONNER, J.T. (1974). " Simulation Experience : World Population/Land Area/Resources/Food ». Futures Conditional, vol. $3, n^{o} 1$, p. 1-3.

CORBO, C. et coll. (1994). Préparer les jeunes au $21^{e}$ siècle : Rapport du groupe de travail sur les profils de formation au primaire et au secondaire. Gouvernement du Québec, Ministère de l'Éducation.

DUCHASTEL, P., CHAMBERLAND, G. et H. BEAULIEU (1989). « Le jeu éducatif à base de connaissances » dans L. Sauvé et R. Viau (dir.). La technologie éducative à la croisée des disciplines, les actes $d u 7^{e}$ symposium $d u$ Conseil interuniversitaire des professeurs en technologie éducative. Mont Sainte-Anne.

DUKE, R.D. et C.L. MATTLEY (1986). « The Effects of Social Structure and Mobility on Attitudes and Behavior in a Simulated Society ». Simulation \& Games, vol. 17, $\mathrm{n}^{\mathrm{o}} 4$, décembre, p. 467-484.

FRIPP, J. (1993). Learning Through Simulations : A Guide to the Design and Use of Simulations in Business and Education. New York : McGraw-Hill.

GAGNÉ, R. (1976). Les principes fondamentaux de l'apprentissage. Montréal : Les éditions HRW.

GARON, D. (1985). La classification des jeux et des jouets : le système ESAR. La Pocatière : Documentor.

GLICKMAN, C.D. (1979). « Problem : Declining Achievement Scores, Solution : Let Them Play!»Phi Delta Kappan. février, p. 454-455. 
GLICKMAN, C.D. (1984). « Play in Public School Settings : A Philosophical Question ». Phi Delta Kappan, février, p. 255271.

GREENBLAT, C.S. et R.D. DUKE (1975). Gaming-Simulation, Rationale Design and Applications, Beverly Hills, CA : Sage Publications.

GREENBLAT, C.S. (1988). Designing Games and Simulations : An Illustrated Handbook. Newbury Park, CA : Sage.

GRUNDFIELD, F.V. (1977). Games of the World. New York: Random Flouse, Ballantine Books.

HEINICH, R., MOLENDA, M. et J.D. RUSSELL (1989). Instructional Media and the New Technologies of Instruction. $3^{\mathrm{e}}$ éd. New York : Wiley, p. 330-353.

HUIZINGA, J. (1988). Homo ludens : essai sur la fonction sociale du jeu. Paris : Gallimard.

JONES, K. (1980). Simulations : A Handbook for Teachers. London : Kogan Page.

JONES, K. (1988). Interactive Learning Events : A Guide for Facilita tors. New York : Nichols Publishing.

KOLB, D.A., RUBIN, I.M. et J.M. OSLAND (1991). Organizational Behauiour : An Experiential Approach. $5^{\mathrm{e}}$ éd. Englewood Cliffs, N.J. : Prentice-Hall.

LEVY, J. (1968). « Differential Perceptual Capacities in Major and Minor Hemispheres » Proceedings of the National Academy of Science, $\mathrm{n}^{\mathrm{o}}$ 61, p. 1151.

MAIDMENT, R. et M.J. BRONSTEIN (1973). Simulation Games. Columbus, $\mathrm{OH}$ : Charles E. Merrill Publishing Co.

NORRIS, D.R. (1986). " External Validity of Business Games ». Simulation \& Games, vol. 17, $\mathrm{n}^{\circ}$ 4, décembre, p. 447-459.

McLEAN, H.W. et M.J. RAYMOND (1976). Design Your Own Game. Lebanon, $\mathrm{OH}$ : The Simulation and Game Association. 
OSBORN, A.F. (1971). L'imagination constructive. Paris : Dunod.

PAIDEIA, I. (1975). « La notion de 'jeu' dans la pédagogie des Jésuites au XVIIe siècle » Revue des sciences humaines, vol. $158, \mathrm{n}^{\circ} 2$, p. 251-265.

RABECQ-MAILLARD, M.M. (1969). Histoire des jeux éducatifs. Paris : Nathan.

RASER, J.R. (1969). Simulation and Society : An Exploration of Scientific Gaming. Boston : Allyn and Bacon.

REINHARTZ, J. et D. VAN CLEAF (1986). Teach-PracticeApply: the TPA Instruction Model, K-8. Washington, D.C. : National Education Association.

RICHEY, R. (1986). « A Proposed Approach to Theory Development in Instructional Design » dans The Theoretical and Conceptual Bases of Instructional Design, New York : Nichols Publishing Co.

ROGERS, C.R. (1976). Liberté pour apprendre? Paris : Dunod.

SAEGESSER, F. (1981). "Simulation-Gaming in the Classroom : Some Obstacles and Advantages ». Simulation and Games, vol. $12, \mathrm{n}^{\mathrm{O}} 3$, p. 281-294.

SANDERS, P. et J.N. YANOUZAS (1985). « Experiential Socialization ». Simulation \& Games, vol. 16, n 1 , p. 71-85.

SCHÜTZENBERGER, A.A. (1981). Le jeu de rôle : connaissance du problème - applications pratiques. Collection formation permanente en sciences humaines, Paris : Éditions ESF Entreprise moderne d'édition.

SCHWARTZMAN, H.B. (1978). Transformations : The Anthropology of Children's Play. New York : Plenum Press.

SPERRY, R.W. (1973). " Lateral Specialization of Cerebral Function in the Surgically Separated Hemispheres » dans F.J. McGuigan et R.A. Schoonover (dir.). The Psychology of Thinking. New York : Academic Press, p. 209-229. 
STADSKLEV, R. (1980). Handbook of Simulation and Gaming in Social Education : Part I - Textbook. Institute of Higher Education Research and Services, University of Alabama.

STEVENS, M.-A. (1978). « Les jeux des écoliers à l'époque de la Renaissance ». Revue interdisciplinaire de philosophie morale et politique/Communication sociale et éducation, p. 53-59.

STOLOVITCH, H. et D. DUPONT (1979). « Le jeu » dans Les actes du colloque sur la pédagogie universitaire. Montréal : Service de pédagogie de l'Université de Montréal, p. 187-190.

STOLOVITCH, H. et S. THIAGARAJAN (1980). Frame Games. Englewood Cliffs, N.J. : Educational Technology Publications.

STOLOVITCH, H. (1989). «Frame Games and Game Chains : A Technology for Interactive Teaching/Learning Communication ». Primer Seminario Interuniversitario. Caracas, Venezuela : Universidad Central de Venezuela.

TAYLOR, J. et R. WALFORD (1978). Learning and the Simulation Game. Milton Keynes : Open University Press.

THIAGARAJAN, S. (1977). "Keep That Delicate Balance ». Simulation/Gaming, vol. 5, $\mathrm{n}^{\mathrm{o}}$ 4, p. 4-8.

TROYKA, L.Q. et J. NUDELMAN (1975). Taking Action : Writing, Reading, Speaking and Listening Through Simulation-Games. Toronto : Prentice-Hall, p. 38-51.

VAN MENTS, M. (1983). The Effective Use of Role-Play : A Handbook for Teachers. London : Kogan Page.

VIAL, J. (1981). Jeu et éducation : les ludothèques. Paris : Presses universitaires de France. 



\section{Index}

A

Alerte à l'environnement $\mathbf{1 0 0}$, 101

B

Bafà Bafà 104

Bateau de sauvetage 85

bingo 119, 120, 121, 123, 159

Bonhomme 98, 99

C

cachette 6

Ça va vous tuer 121, 122

Chaturanga 9

Close Encounters of the Difficult Kind 92

Conservation Crisis 100

Cribbage 37

D

dames 6, 35

Diplomacy 40, 53

Donjons et dragons 73
É

échecs $6,7,9,10,11,35,169$ échecs chinois 9

G

ghetto 82

go 11,35

J

jeu de l'oie 13, 14, 37

jeu du chat et de la souris 6

K

kriegspiels 15

L

Labyrinthe 41, 51

Labyrinthe Master 51

Le jeu de la naissance 38,49

Le lièvre et la tortue 40,50 
Jeu, simulation et jeu de rôle

Le nouveau jeu des vertus récompensées et des vices punis ou le triomphe de la vertu 14

\section{M}

Mancala 7

Meurtres et mystères 73

Moksha-patamu 12

Monopoly 33, 36, 37, 40

N

Narcisse et Pivoine sur le chemin des floralies 38,39

$\mathbf{R}$

Rencontres du $13^{\mathrm{e}}$ type 92, 94, 95, 96

Répartition 65, 66, 67, 68

Risk 40, 41
S

Sagix 119, 120, 121

Sang bon sens 42,52

Scrabble 35, 48, 160

Senet 7

serpents et échelles $11,36,37$

SimCity 200069

'Smath 48

T

They Shoot Marbles, Don't

They ? 57, 70

tic-tac-toe 121, 122, 123

$\mathbf{U}$

Uno 160

W

Wari 7 

$/ V \sqrt{a} \cdot$ Cap-Saint-Ignace

S Sainte-Maric (Beauce)

M Québec, Canada

"LIMPRIMEUR" 